\title{
ACHIEVING DATA PRIVACY THROUGH VIRTUAL UPDATES
}

by

Lechen $\mathrm{Li}$

\author{
A thesis submitted to \\ the Faculty of Graduate Studies and Research \\ in partial fulfillment of \\ the requirements for the degree of \\ MASTER OF SCIENCE \\ School of Computer Science \\ at \\ CARLETON UNIVERSITY
}

Ottawa, Ontario

January, 2011

C) Copyright by Lechen Li, 2010 
Library and Archives

Canada

Published Heritage Branch

395 Wellington Street

Ottawa ON K1A ON4

Canada
Bibliotheqque et

Archives Canada

Direction du

Patrimoine de l'édition

395 , rue Wellington

Ottawa ON K1A ON4

Canada
Your file Votre référence

ISBN: 978-0-494-79595-8

Our file Notre référence

ISBN: 978-0-494-79595-8
NOTICE:

The author has granted a nonexclusive license allowing Library and Archives Canada to reproduce, publish, archive, preserve, conserve, communicate to the public by telecommunication or on the Internet, loan, distribute and sell theses worldwide, for commercial or noncommercial purposes, in microform, paper, electronic and/or any other formats.

The author retains copyright ownership and moral rights in this thesis. Neither the thesis nor substantial extracts from it may be printed or otherwise reproduced without the author's permission.
AVIS:

L'auteur a accordé une licence non exclusive permettant à la Bibliothèque et Archives Canada de reproduire, publier, archiver, sauvegarder, conserver, transmettre au public par télécommunication ou par l'Internet, prêter, distribuer et vendre des thèses partout dans le monde, à des fins commerciales ou autres, sur support microforme, papier, électronique et/ou autres formats.

L'auteur conserve la propriété du droit d'auteur et des droits moraux qui protège cette thèse. $\mathrm{Ni}$ la thèse ni des extraits substantiels de celle-ci ne doivent être imprimés ou autrement reproduits sans son autorisation.
In compliance with the Canadian Privacy Act some supporting forms may have been removed from this thesis.

While these forms may be included in the document page count, their removal does not represent any loss of content from the thesis.
Conformément à la loi canadienne sur la protection de la vie privée, quelques formulaires secondaires ont été enlevés de cette thèse.

Bien que ces formulaires aient inclus dans la pagination, il n'y aura aucun contenu manquant. 


\section{Contents}

List of Tables $\quad$ v

List of Figures vi vi

$\begin{array}{lll}\text { Abstract } & \text { vii }\end{array}$

Acknowledgements viii

$\begin{array}{lll}\text { Chapter } 1 & \text { Introduction } & 1\end{array}$

11 Problem Statement and Contributions $\quad$. $\quad . \quad$. 5

$\begin{array}{llr}\text { Chapter } 2 & \text { Preliminaries } & 8\end{array}$

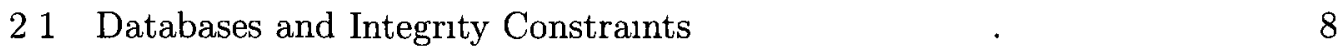

211 The IsNull Predicate 14

212 Primary Keys . . . . . $\quad$. 14

22 Query Answering in Databases with Null Values 16

23 Database Repairs and Consistent Query Answernng . . . . . . 20

2.4 Views . . . . . . . . . . . . . . . . . 21

Chapter $3 \quad$ Null Semantics for SQL Queries and ICs 23

31 Interpretations of Null Values . . . $\quad \ldots \quad \ldots \quad \ldots \quad \ldots$

32 Null Interpretation in SQL queries . . . . . . . . 26

33 IC Satisfaction in Databases with Null Values _ . . . . . 31

33.1 Semantics of Unique Constrants . . $\quad \ldots \quad \ldots \quad$. . 32

3 3.2 Semantics of Primary Key Constraints ․ $\quad 34$

333 Semantıcs of Foreıgn Key Constraints . . . . . . . 35 
4.1 Secrecy Instances of Incomplete Databases . . . . . . . . 39

42 Privacy Preserving Query Answernng . . . . . . . . . . . . 47

43 Summary . . . . . . . . . . . . . . . . . 52

Chapter $5 \quad$ Secrecy Logic Programs 53

51 Optımızing Query Evaluation from Secrecy Programs . . . . . 62

511 Relevant Predicates . . . . . . . . . . . 63

51.2 Relevant Facts . . . . . . . . . . . . . . . 66

$\begin{array}{lllll}52 & \text { Summary } & \ldots & \text {. } & 67\end{array}$

$\begin{array}{llr}\text { Chapter } 6 & \text { Experiments } & 69\end{array}$

$\begin{array}{lll}61 & \text { Prelıminaries } & 69\end{array}$

62 Experimental Setup _ . . . $\quad$. $\quad 74$

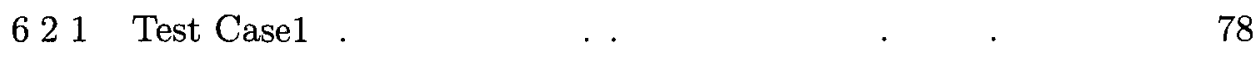

622 Test Case2 . . . . . . . . . $\quad$. 79

623 Test Case3 . 80

63 Results . . . . . . . . . . . 80

$\begin{array}{lll}\text { Chapter } 7 & \text { Discussion } & \mathbf{8 3}\end{array}$

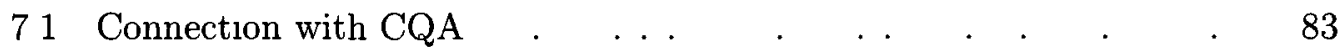

72 Connection with Data Cleaning _. . . . . . . 84

73 Related Work . . . . . . . . . . . . . . 89

$\begin{array}{lll}\text { Chapter } 8 & \text { Conclusions and Future Work } & 92\end{array}$

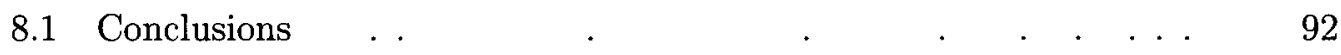

82 Future Work $\ldots \ldots \ldots$. . . . . . . . . 93 
Appendix A Optimized Secrecy Programs 


\section{List of Tables}

Table 3.1 Truth Table for Conjunction . . . . . . . . . . . . . 25

Table 32 Truth Table for Disjunction . . . . . . . . . . . 25

Table 3.3 Truth Table for Negation $\quad \ldots \quad \ldots \quad \ldots \quad$. . . . . . 25

Table 3.4 Employee Table . . . $\quad$. . . . . . . . . 27

Table 35 Dept Table $\quad \ldots \quad$. $\quad 28$

Table 5.1 Annotatıon Constants $\ldots \quad \ldots \quad \ldots \quad \ldots \ldots$

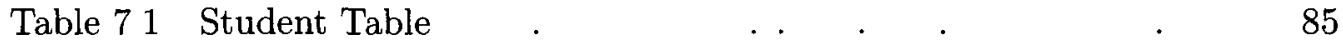

Table 72 Match Relevancy $\quad \ldots \quad \ldots \quad$. $\quad$. $\quad$. . . . . . . 88

Table 73 Non-Match Relevancy . . . . 88

$\begin{array}{llll}\text { Table } 74 & \text { Match Matrix } & \text {. . } & 89\end{array}$ 


\section{List of Figures}

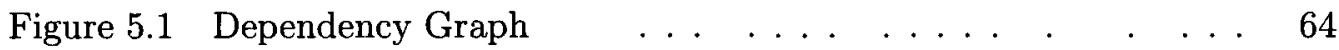

Figure 6.1 Architecture of Query Evaluation _ . . . . . . 70

Figure 6.2 Execution Time for $\mathcal{Q}_{1} \quad \ldots \ldots \ldots \ldots$. . . . . . 81

Figure 63 Execution Time for $\mathcal{Q}_{2} \quad$. $\quad . \quad$. . . . $\quad . \quad$. $\quad 81$

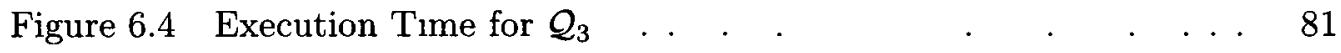




\section{Abstract}

There may be sensitive information in a relational database that we want to keep hidden from a user of group thereof. The sensitive data is characterized as the contents of a view Whenever the user poses queries about these secrecy views, the set of answers either becomes empty or contains tuples with only null values This is achieved through updates of the given instance Since the database is not expected to be physically changed to produce this result, it is only virtually and minimally updated, and those changes are reflected in the view contents Virtual updates are based on the use of null values as used in the SQL standard The different ways to update the underlyıng database are specıfied as the models of logic programs with stable model semantics The programs become the basis for the computation of the secret answers to queries, $1 \mathrm{e}$ those that do not reveal the sensitive information More precisely, secret answers can be computed by evaluating queries against the programs, e g. using the disjunctive datalog system (DLV). An optimization approach 1s developed for evaluatıng queries, in such way only relevant data facts and program rules are involved in query evaluation. 


\section{Acknowledgements}

It is a pleasure to thank the many people who made this thesis possible

First of all, I would like to thank Professor Leopoldo Bertossı, my supervisor, for his constant encouragement, advice, support, lots of good ideas throughout my thesis He has helped me a lot through all the stages of the writing of my thesis Without his instructive advice and suggestion, the completion of this thesis would not have been possible

I am also grateful to the School of Computer Science at Carleton University for providing us with a good environment and facilities to complete this research I'd like to thank all my professors who have helped me to broaden my view and knowledge.

Last but not least, I would like to give special thanks to all my family for supportıng me and being there whenever help was needed

viii 


\section{Chapter 1}

\section{Introduction}

Database management systems allow for massive storage of data, which can be easily and efficiently accessed and manipulated However, at the same time, the problems of data privacy are increasingly important. For example, for commercial or legal reasons, administrators of sensitive information may not want or be allowed to release certain portions of the data It becomes crucial to address database privacy issues.

In this scenario, authorization is required. More precisely, certain users can have access to only certain portions of a database Hopefully, what a partıcular user (or class of them) is allowed or not allowed to access should be specified in a declarative manner Currently, authorization mechanisms in commercial database management systems are at the level of columns, the whole relations, or on views But there are many situations that demand a very fine-grained level, such as at the level of individual tuples or cells For example, imagine a company's database that included Information about an employee's health If that employee has a disease, this must be kept as a secret Otherwise, this health information could be publicly released

A possible way to solve tuple-based access control is to create views for each user, or class of them Those views contain only selected tuples of a database, those it allows a user (or class of them) to access. However, this approach is not practical since there may be a large number of users. Most of information systems in practice ignore tuple-based access control at the database level, and delegate this problem to the application level In other words, application programs are used to ensure 
appropriate access rather than databases Although widely used, this approach has serval disadvantages For example, lots of source code has to be changed if any policy 1s changed. In addition, by putting access control into the application, you need to duplicate the access logics in each application that accesses the database, which will increase the cost and complexity of development Therefore, it is better to address access control at the database level.

Some recent papers $[37,44]$ approach fine-grained access control in the database in terms of authorization views View-based data privacy usually approaches the problem by specifying which views a user $2 s$ allowed to access. For example, when the database receives a query from that user, it checks if the query can be answered using those views alone. More precisely, if the query can be rewritten in terms of the views [37]. If no complete rewritıng is possible, the query is rejected In [44] the problem about the existence of a conditional rewriting is investıgated, 1 e relative to an instance at hand

Our approach to the data protection problem is based on specifications of what users are not allowed to access through query answers, which is quite natural Data owners usually have a more clear picture of the data that is sensitive rather than about the data that can be publicly released Dealing with this problem as "the complement" of the problem formulated in terms of authorization views is not natural, and not necessarıly easy, especially consıderıng that complements of database views would be involved $[27,28]$.

According to our approach, the information to be protected is declared as a view, a secrecy view, or a collection of them Each user or class of them may have associated a set of secrecy views. When a user poses a query on the database, the system will virtually update some of the attributes values in terms of a fixed set of secrecy views In each of the resulting updated instances, the extension of secrecy views either becomes empty or contains tuples showing only null values. Then, the original 
query is posed to the updated instances, and is answered as usual. In this way, the system will return answers to the query that do not reveal the secret data In this work, we consider updates that modify attribute values assigning null values.

Example 1.1. Consider the following relational database $D$

\begin{tabular}{c|c|c|c|}
\hline Marks & StudentID & CourseID & Mark \\
\hline \multirow{6}{*}{001} & 01 & 56 \\
001 & 02 & 90 \\
002 & 02 & 70 \\
\cline { 2 - 4 }
\end{tabular}

The following secrecy view $V_{s}$ on the database specifies that a student with her course mark must be kept secret when the mark is less than 60 .

$$
V_{s}(s \imath d, c \imath d, \text { mark }) \leftarrow \operatorname{Marks}(s \imath d, c \imath d, \text { mark }), \text { mark }<60^{1}
$$

Now, a user of the database wants to see students' marks, using the following query

$$
Q(s \imath d, c \imath d, m a r k) \leftarrow \operatorname{Mark}(s \imath d, c \imath d, m a r k)
$$

Through this query the user can obtain the first record $\operatorname{Mark}(001,01,56)$, which is sensitive information A way to solve this problem consists in vrrtually updating the base relation in terms of view definitions, in such a way that the secret information, i e the extension of the secrecy view, can not be revealed to the user Here, in order to protect the tuple $\operatorname{Mark}(001,01,56)$, the new instance $D^{\prime}$ is obtained by virtually updating the original instance, changing the attribute value 56 to $\mathrm{null}$. The virtually updated instance is $D^{\prime}$ :

\footnotetext{
${ }^{1}$ We use Datalog notation for view definitions and queries
} 


\begin{tabular}{c|c|c|c|}
\hline Marks & StudentID & CourseID & Mark \\
\hline \multirow{1}{*}{001} & 01 & null \\
001 & 02 & 90 \\
002 & 02 & 70 \\
\hline
\end{tabular}

Now, by posing the query

$$
Q_{1}(s \imath d, c \imath d, \text { mark }) \leftarrow \operatorname{Mark}(s \imath d, c \imath d, \text { mark }), \text { mark }<60,
$$

which corresponds to the secrecy view definition, to the "updated" database $D^{\prime}$, the user gets an empty answer, because the comparison of null with any value will be evaluated as unknown. Simlarly, query (1 11$)$ will get the first tuple with null instead of 56 The user cannot obtain the mark 56 by combining any answers obtained through other queries on $D^{\prime}$

Hidıng sensitive information is one of the concerns Another one is about still providing as much information as possible to the user In consequence, the virtual update has to be minimal in some sense, whle still doing its job of protecting data In the previous example, we might consider virtually deleting the whole tuple $\operatorname{Marks}(001,05,56)$, to protect secret information, but we may lose some useful information, like the student ID and the course ID Furthermore, the user should not be able to guess the protected information by combing information obtained from different queries

As illustrated above, null values will be used to virtually update the database instance As expected, null values have received attention from the database community $[43,41,24]$. Null values may have several interpretations, e $\mathrm{g}$ as a replacement for a real value that is non-existent, missing, unknown, inapplicable, etc Several formal semantics have been proposed for them. Furthermore, it is possible to consider different, coexisting null values. In this work, we will use a single null value, denoted 
as above and in the rest of this thesis, by null. Furthermore, we will treat null as the NULL in SQL relational databases Since the SQL standard does not provide a precise, formal semantics for NULL, we will adopt here the formal, logical reconstruction of SQL nulls proposed in [12]. It captures the semantics of the SQL NULL that are relevant for our work on privacy, namely integrity constraint satisfaction and query answering. This makes our approach to secrecy compatıble with and implementable on top of commercial DBMSs

\subsection{Problem Statement and Contributions}

The goal of this research is to develop methods to retrieve answers for first-order conjunctive queries that do not reveal any secret data from relational databases In most practical cases, databases always contain null values So, we will put special interest on databases with null values The key features of our research are the following

1. The notion of secrecy views is introduced. Basically, the sensitive information is protected via a fixed set of secrecy views It is associated to a particular user or class of them We have restricted ourselves mainly to the case of conjunctive secrecy views The following is an example of a conjunctive secrecy view.

$$
V_{s}(s \imath d, c \imath d, \text { mark }) \leftarrow \operatorname{Marks}(s \imath d, c \imath d, \text { mark }), \text { mark }<60
$$

Here, the secrecy view is specified using a Datalog rule In general, we will use Datalog notation for views and queries.

2. In this thesis, we introduce null-based virtual updates on an instance, to keep the secrecy view extensions secret The semantics of secrecy in the presence of null values that we provide is model-theoretıc, in the sense that the possible admissible instances after the update, the so-called secrecy instances, are characterızed and specified. On a secrecy instance, the extensions of the secrecy 
views contain only tuples with null or become empty Furthermore, the secrecy instances do not depart from the original instance by more than what is needed to protect the secret data. So, they minimally differ in a precise sense from the original instance.

3. The semantics of secret answers to a query is presented. Those answers are invariant under the class of secrecy instances. More precisely, a ground tuple $\bar{t}$ to a first order query $\mathcal{Q}(\bar{x})$ is a secret answer from instance $D$, if it is an answer to $\mathcal{Q}(\bar{x})$ in every possible secrecy instance for $D$

4 In this thesis, we also show that our null-based semantics can be captured in terms of disjunctive logic programs with extra annotation constants, by establishing a correspondence between the secrecy instances of a relational database and the stable models of the program The logic program can be used to specify the secrecy instances of the original database and to obtain secret answers The basic idea behind the logic programming-based approach is that, since we need to reason simultaneously with all the secrecy instances of a database, we have to succinctly specify the class of secrecy instances Then, different reasoning tasks could be performed, in particular, computation of secret answers to queries We also show how to obtain a reduced secrecy view and a subset of sources for a query.

5 We also develop optimization techniques to reduce the amount of data involved in the computation of secret answers to queries, in such a way that only a subset of database facts and program rules are involved in query evaluation.

6 We discuss several additional issues in relation to our approach to privacy There are some interesting connections between this work and consistent query answering $[5,8]$ The secrecy instances can be seen as repairs of the original 
instance that enforce semantic constraints on a given instance. These repairs minimally differ from the given instance under a repair semantics that is based on attribute updates with null. In this sense, secret query answering becomes a form of consistent query answering (CQA).

7 In additıon, IBM InfoSphere Master Data Management Server (MDM) provides a mechanism, named Suspect Duplicate Processing, to keep a single view of the customer information Our privacy approach could make use of this mechanism to identify or detect duplicates for improving data quality

This thesis is structured as follows Chapter 2 introduces basic definitions and concepts needed in the later chapters Chapter 3 discusses how null values are treated in the different DBMSs Chapter 4 presents a precise semantıcs for secrecy instances and secret answers Chapter 5 describes the specification of secrecy instances via logic programs and how to use them to compute secret answers to first-order conjunctive queries Chapter 6 provides experimental results on varıous scenarios of user querıes Chapter 7 discuses several additıonal issues with respect to the previously introduced semantics, including connectıon with CQA and duplicate detection Chapter 8 presents some conclusions and future work. 


\section{Chapter 2}

\section{Preliminaries}

In this chapter we recall basic concepts of relational databases. We also introduce the database repairs and null-value semantics presented in [11], which is the underlying semantics that will be used in the rest of this work.

\subsection{Databases and Integrity Constraints}

In the context of relational databases, we assume that we have a fixed relational schema $\Sigma=(\mathcal{U}, \mathcal{R}, \mathcal{B})$, where $\mathcal{U}$ is the possibly infinite database domain, such that null $\in \mathcal{U}, \mathcal{R}$ is a fixed set of database predicates say, $\mathcal{R}=\left\{R_{1}, R_{2}, R_{3}, \quad\right\}$, where each relation $R$ has an a finite, ordered set of attributes $\mathcal{A}_{R}$, and $\mathcal{B}$ is a fixed set of bultin predicates, hke comparison predicates, e g. $>,<,=R[\imath]$ denotes the attribute in position $\imath$ of predicate $R \in \mathcal{R}$. For $A \subseteq \mathcal{A}_{R}, R[A]$ denotes predicate $R$ projected on the attributes in $A$

The schema determines a language $\mathcal{L}(\Sigma)$ of first-order predicate logic A database instance $D$ compatible with $\Sigma$ can be seen as a finte collection of ground atoms (or tuples) of the form $R\left(c_{1}, \quad, c_{n}\right)$, where $R \in \mathcal{R}$ and the $c_{1}, \quad, c_{n}$ are constants in $\mathcal{U}$. Bult-in predicates have a fixed extension in every database instance.

A query is a first-order formula over language $\mathcal{L}(\Sigma)$ In this research we restrict ourselves to the class of conjunctive queries

Definition 1. A conjunctive query $\mathcal{Q}(\bar{x})(\mathrm{CQ})$ is of the form

$$
\exists \bar{y}\left(\bigwedge_{\imath=1}^{n} R_{\imath}\left(\bar{x}_{\imath}, \bar{y}_{\imath}\right) \wedge \varphi\right)
$$


where $R_{\imath} \in \mathcal{R}, \varphi$ is a conjunction of built-ins whose variables appear in the $R_{\imath} \mathrm{s}$, and $\bar{x}=\bigcup_{\imath} \bar{x}_{\imath}$ which are the free variables of the query If the query does not have free variables, 1.e. $\bar{x}$ is the empty set, it is called a boolean query

Conjunctive queries can also be written as Datalog rules The following is Datalog notation for the query $(2.1)$ :

$$
\operatorname{Ans}(\bar{x}) \leftarrow R_{1}\left(\bar{x}_{1}, \bar{y}_{1}\right), \quad, R_{n}\left(\bar{x}_{n}, \bar{y}_{n}\right), \varphi
$$

where $A n s$ is an intensional relation symbol, and the head $A n s(\bar{x})$ represents the results of the query $\mathcal{Q}(\bar{x})$

Definition 2. Given a database instance $D$ without null, a tuple of constants $\bar{t}$ is an answer to a query $\mathcal{Q}(\bar{x})$ of the form (21) in $D$ iff $D \vDash \mathcal{Q}(\bar{t})$, a $\mathcal{Q}(\bar{x})$ becomes true in $D$ when the variables are replaced by the corresponding constants in $\bar{t}$ If $\mathcal{Q}$ is a sentence (boolean query), then yes is an answer iff $D \vDash \mathcal{Q}$ Otherwise, the answer is no

Example 2.1. Consider the database $D=\{P(1,1), Q(1)\}$. The query $\mathcal{Q}_{1}: P(1,1)$ has yes as an answer. Query $\mathcal{Q}_{2}(x): \exists y(P(x, y) \wedge Q(y))$ has 1 has an answer

With the purpose of answering first-order queries in a database with null, the notıon of query answering will be modıfied (cf Sectıon 2.2).

Integrity Constraints (ICs) have been considered in the relational databases for adding semantıcs, and ensuring accuracy and consistency of data. The most common type of constraints are functional dependencies and inclusion dependencies.

A functional dependency [4] (FD) over a database schema $\Sigma$ is an expression of the form $R: X \rightarrow Y$, where $R \in \mathcal{R}$ and $X, Y$ are sets of attributes associated to $R$. 
A relation $D$ over $\Sigma$ satisfies $X \rightarrow Y$, denoted by $D \vDash X \rightarrow Y$, if for any two tuples that have the same values in the attributes in $X$, also have the same values in the attributes in $Y$ A set of attributes $X$ is a candidate key of a relation $R$ if for every attribute $Y$ in $R$, it holds that $R \cdot X \rightarrow Y$, and no subset of $X$ has this property One of the candidate keys can be chosen as the primary key (PK).

An inclusion dependency [4] (IND) over a database schema $\Sigma$ is an expression of the form $R[X] \subseteq S[Y]$, where $R, S$ are (possibly identical) relation names in $\Sigma$, and $X, Y$ are sets of attributes from $R$ and $S$, respectively. An instance $D$ of $\Sigma$ satisfies $R[X] \subseteq S[Y]$, denoted by $D \vDash R[X] \subseteq S[Y]$, if for each tuple $\bar{r}$ in $D(R)^{\mathbf{1}}$, there exists a tuple $\bar{s}$ in $D(S)$, such that $\bar{s}[Y]=\bar{r}[X]$ An inclusion dependency is said to be full If $X=\mathcal{A}_{R}$, and partıal if $X \varsubsetneqq \mathcal{A}_{R}$. A foreign key constraint is an inclusion dependency $S[Y] \subseteq R[X]$, where $X$ is the primary key of $R$. More generally,

Definition 3. [12] An integrity constraints is a sentence of the form

$$
\forall \bar{x}\left(\bigwedge_{\imath=1}^{n} P_{\imath}\left(\bar{x}_{\imath}\right) \rightarrow \exists \bar{z}\left(\bigvee_{\jmath=1}^{m} Q_{\jmath}\left(\bar{y}_{\jmath}, \bar{z}_{\jmath}\right) \vee \varphi\right)\right)
$$

where $P_{\imath} . Q_{\jmath} \in R, \bar{x}=\bigcup_{\imath=1}^{m} \bar{x}_{\imath}, \bar{z}=\bigcup_{\jmath=1}^{n} \bar{z}_{\imath}, \bar{y}_{\imath} \subseteq \bar{x}, \bar{x} \cap \bar{z}=\varnothing, \bar{z}_{\imath} \cap \bar{z}_{\jmath}=\varnothing$ for $\imath \neq \jmath$ Here, $\varphi$ is a disjunction of bult-in predicates from $\mathcal{B}$, whose variables appear in the antecedent of the implication. We will assume that there exists a propositional atom false $\in \mathcal{B}$ that is always false in a database.

Definition 4. [12] A unversal integrity constraint (UIC) is an sentence in $\mathcal{L}(\Sigma)$ of the form:

$$
\forall \bar{x}\left(\bigwedge_{\imath=1}^{n} R_{\imath}\left(\bar{x}_{\imath}\right) \longrightarrow\left(\bigvee_{j=1}^{m} Q_{\jmath}\left(\bar{y}_{\jmath}\right) \vee \varphi\right)\right)
$$

That is a formula of the form (23), without existential quantifiers

\footnotetext{
${ }^{1} D(R)$ denotes the extension of predicate $R \in \mathcal{R}$ in an instance $D$
} 
Definition 5. [12] A referentıal integrity constrant (RIC) is a sentence in $\mathcal{L}(\Sigma)$ of the form:

$$
\forall \bar{x}(P(\bar{x}) \rightarrow \exists \bar{z} Q(\bar{y}, \bar{z}))
$$

That is a formula of the form (23), with $m=n=1$, without a $\varphi, \bar{y} \subseteq \bar{x}$, and $P, Q \in \mathcal{R}$

The class of ICs of the form (2.3) contains most of the ICs commonly found in database practice. Functional dependencies can be expressed by several implications of the form (2.4), each of them with a single equality in the consequent. Partial inclusion dependencies are RICs, and full inclusion dependencies are UICs A denıal constraınt can be expressed as $\forall \bar{x}\left(\bigwedge_{\imath=1}^{n} R_{\imath}\left(\bar{x}_{\imath}\right) \rightarrow\right.$ false $)$

Example 2.2. Consider a database schema $\Sigma=\{$ Employee $($ EId, Name, Dept $)$, $\operatorname{Dept}(\operatorname{DId}$, Name $)\}$ The following are ICs

(a) The functional dependency (FD) Employee : EId $\rightarrow$ Name, expressed in $L(\Sigma)$ by $\forall e \imath d \forall n 1 \forall n \mathscr{L}($ Employee $(e \imath d, n 1) \wedge$ Employee $(e \imath d, n \mathscr{L}) \rightarrow n 1=n \mathscr{2})$.

(b) The inclusion dependency (IND) Employee [Dept] $\subseteq \operatorname{Dept}[\operatorname{DeptID}]$, expressed by $\forall e \imath d \forall n \forall \operatorname{dept}(\operatorname{Employee}(e \imath d, n, \operatorname{dept}) \rightarrow \exists \operatorname{dnDept}(\operatorname{dept}, d n))$.

We consider a fixed finite set $I C$ of ICs of the form (2 3) A database $D$, possibly with null values, is consistent with respect to $I C$ if it satisfies the given set $I C$; otherwise, we say $D$ is inconsistent The semantics of constraint satisfaction in presence of null values we consider is the one defined in $[12,11]$ In order to present it, we need to introduce some concepts.

Definition 6. [12] For an IC $\psi \in \mathcal{L}(\Sigma)$ and a term $t$ (i e. a variable or a domain constant), $\operatorname{pos}^{R}(\psi, t)$ is the set of positions in predicate $R \in \mathcal{R}$ where $t$ appears in $\psi$. 
The set of relevant attributes for an IC $\psi$ of the form $(23)$ is $\mathcal{A}(\psi)=\left\{R[\imath] \mid x\right.$ is a variable present at least twice in $\mathcal{V}(\psi)$, and $\left.\imath \in \operatorname{pos}^{R}(\psi, x)\right\} \cup$ $\left\{R[\imath] \mid c\right.$ is a constant in $\psi$ and $\left.\imath \in \operatorname{pos}^{R}(\psi, c)\right\}$ where $R[\imath]$ denotes a position in relation $R$

The relevant attributes include the attributes needed to check the satısfaction of the constraints, e.g the attributes in joins, in bult-ıns, etc.

Definition 7. [12] Given a set of attributes $\mathcal{A}$, and a predicate $R \in \mathcal{R}$, we denote by $R^{\mathcal{A}}$ the predicate $R$ restricted to (or projected onto) the attributes in $\mathcal{A} D^{\mathcal{A}}$ denotes the database $D$ with all its database atoms projected onto the attributes in $\mathcal{A}, 1 \mathrm{e}$ $D^{\mathcal{A}}=\left\{R^{\mathcal{A}}\left(\Pi_{\mathcal{A}}(\bar{t})\right) \mid R(\bar{t}) \in D\right\}$, where $\Pi_{\mathcal{A}}(\bar{t})$ is the projection on $\mathcal{A}$ of tuple $\bar{t} D^{\mathcal{A}}$ has the same underlying domain $\mathcal{U}$ as $D$

Example 2.3. Consider a IC $\psi$ of the form (23) $\forall x(P(x) \rightarrow \exists z Q(x, z))$, and the following database instance

\begin{tabular}{c|c|}
\hline$P$ & $A$ \\
\hline & $a$ \\
& null
\end{tabular}

\begin{tabular}{c|c|c|}
\hline$Q$ & $A$ & $B$ \\
\hline & $a$ & 1 \\
& $b$ & 2 \\
\hline
\end{tabular}

Here $x$ appears twice in $\psi$, therefore $\mathcal{A}(\psi)=\{P[1], Q[1]\}$ The value in the attribute $B$ is not relevant to check satisfaction of the constraint $\psi$ This makes sense, since we just want values in the attribute $A$ of $P$ also to appear in the attribute $A$ of $Q$, which is equivalent to checking if $\forall x\left(P^{\mathcal{A}(\psi)}(x) \rightarrow Q^{\mathcal{A}(\psi)}(x)\right)$ is satisfied by $D^{\mathcal{A}(\psi)}$, where $D^{\mathcal{A}(\psi)}$ is

\begin{tabular}{c|c|}
\hline$P^{\mathcal{A}(\psi)}$ & $A$ \\
\hline & $a$ \\
& null \\
\hline
\end{tabular}

\begin{tabular}{c|c|}
\hline$Q^{\mathcal{A}(\psi)}$ & $A$ \\
\hline & $a$ \\
& $b$
\end{tabular}


In a commercial SQL DBMS, a constraint is satisfied if any of the relevant attributes has a null value or the constraint is satisfied in the traditional way. More formally,

Definition 8. [12] A constraint $\psi$ of the form (2.3) is satisfied in the database instance $D$, denoted $D \vDash_{N} \psi$, iff $D^{\mathcal{A}(\psi)} \vDash \psi^{N}$, where $\psi^{N}$ is

$$
\forall \bar{x}\left(\bigwedge_{\imath=1}^{m} R_{i}^{\mathcal{A}(\psi)}\left(\bar{x}_{\imath}\right) \rightarrow\left(\bigvee_{v_{\jmath} \in(\mathcal{A}(\psi) \cap \bar{x})} v_{\jmath}=n u l l \vee \exists \bar{z}\left(\bigvee_{\jmath=1}^{n} Q_{\jmath}^{\mathcal{A}(\psi)}\left(\bar{y}_{\jmath}, \bar{z}_{\jmath}\right) \vee \varphi\right)\right)\right)
$$

where $\bar{x}=\cup_{z=1}^{m} \bar{x}_{\imath}$, and $\bar{z}=\cup_{j=1}^{n} \bar{z}_{j} \quad$ Here, $D^{\mathcal{A}(\psi)} \vDash \psi^{N}$ refers to the classical first-order satisfaction, where null is treated as any other constant in the domain

We can see from Definition 8 that there are basically three cases for an integrity constraint of the form (2 3) satisfaction· (a) If null is in any of the relevant attributes in the antecedent, then the constraint is satısfied, (b) At least one of the conjunctive clauses has to be true. Theses clauses can be checked by the second disjunction in the consequent of formula (2.6), treating null as any other constant, (c) If the bult-in formula $\varphi$ is satisfied, then the constraint is satisfied.

Example 2.4. (example 2.3 continued) In order to check if $D \vDash_{N} \psi$, we need to check $D^{\mathcal{A}(\psi)}=\psi^{N}$, with $\psi^{N} \quad \forall x\left(P^{\mathcal{A}(\psi)}(x) \rightarrow x=n u l l \vee Q^{\mathcal{A}(\psi)}(x)\right)$. For $x=a$, it holds that $D^{\mathcal{A}(\psi)} \vDash P^{\mathcal{A}(\psi)}(a)$ Since $a$ is not a null value, we need to check if $D \vDash Q^{\mathcal{A}(\psi)}(a)$, which is true in this case Now, for $x=n u l l, D^{\mathcal{A}(\psi)} \vDash P^{\mathcal{A}(\psi)}($ null $)$, and null $=$ null, so the constraint is satisfied 


\subsubsection{The IsNull Predicate}

There is a very important constraint widely used in DBMSs. the NOT NULL constraint (NNC), which prevents certain attributes from taking the value null

Definition 9. [12] A NOT NULL-constraint (NNC) is a denial constraint of the form.

$$
\forall \bar{x}\left(P(\bar{x}) \wedge \operatorname{IsNull}\left(x_{\imath}\right) \rightarrow \text { false }\right),
$$

where $x_{\imath} \in \bar{x}$ is in the position of the attribute that can not take null values. Here,

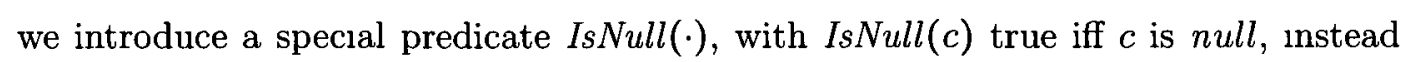
of using the built-in comparison atom $c=n u l l$, because in traditional DBMSs this equality would be always evaluated as unknown.

Notıce that a NNC is not of the form (23), because it contains the special predicate IsNull

Definition 10. [11] A NNC $\psi$ of the form (2.7), is satısfied by a database $D$ with null, denoted $D \vDash_{N} \psi$, iff

$$
D \vDash \forall x\left(\left(P(\bar{x}) \wedge x_{\imath}=n u l l\right) \rightarrow \text { false }\right),
$$

with null treated as any other constant.

Example 2.5. Consider the NNC $\psi: \forall x y(P(x, y) \wedge I s N u l l(x) \rightarrow$ false $)$ This constraint is satisfied if $D \vDash \forall x y(P(x, y) \wedge x=n u l l \rightarrow$ false $)$

\subsubsection{Primary Keys}

In [11], they also propose a precise formalization in first-order logic of the notıons of primary key and unique key satisfaction in databases that conform to the SQL standard SQL-2003. 
Definition 11. [11] Given a predicate $R\left(x_{1}, ., x_{n}\right)$ and its primary key $\{R[1], .$, $R[m]\}^{2}$, the primary key can be logically expressed as the following set of formulas:

$$
\begin{aligned}
& \forall \bar{x} \bar{y}\left(R\left(x_{1}, \ldots, x_{m}, x_{m+1}, \ldots, x_{n}\right) \wedge R\left(x_{1}, \ldots, x_{m}, y_{m+1}, \ldots, y_{n}\right) \rightarrow x_{\jmath}=y_{\jmath}\right), \\
& \quad \text { for } \jmath=m+1, \ldots, n . \\
& \forall \bar{x} \bar{y}\left(R\left(x_{1}, ., x_{m}, x_{m+1}, \ldots, x_{n}\right) \wedge R\left(x_{1}, \ldots, x_{m}, y_{m+1}, \ldots, y_{n}\right) \wedge I s N u l l\left(x_{\jmath}\right)\right. \\
& \left.\quad \rightarrow \operatorname{IsNull}\left(y_{\jmath}\right)\right), \quad \text { for } \jmath=m+1, ., n . \\
& \forall \bar{x} \bar{y}\left(R\left(x_{1}, \quad, x_{m}, x_{m+1}, ., x_{n}\right) \wedge \operatorname{IsNull}\left(x_{\jmath}\right) \rightarrow \text { false }\right), \quad \text { for } \jmath=1, ., m
\end{aligned}
$$

The third set of rules, are NNCs for all the attributes in the key. A unique key can be logically expressed by using only the first two set of rules.

Example 2.6. Consider a schema with relations $R(X, Y)$, with primary key $(\mathrm{PK})$ $R[1]$. The following database instance $D$ violates the PK.

\begin{tabular}{c|c|c|}
\hline$R$ & $X$ & $Y$ \\
\hline & $a$ & $b$ \\
$a$ & null \\
\cline { 2 - 3 }
\end{tabular}

By Definition 11, the PK can be written as $\forall x y z(R(x, y) \wedge R(x, z) \rightarrow y=z), \forall x y z(R(x, y) \wedge$ $R(x, z) \wedge I s N u l l(y) \rightarrow \operatorname{IsNull}(z))$, and $\forall x y(R(x, y) \wedge I s N u l l(x) \rightarrow$ false $)$.

In order to check if $D$ satisfies the PK, we need to check the following three formulas*

$$
\begin{gathered}
D \vDash \forall x y z(R(x, y) \wedge R(x, z) \rightarrow x=n u l l \vee y=n u l l \vee z=n u l l \vee y=z) . \\
D \vDash \forall x y z(R(x, y) \wedge R(x, z) \wedge y=n u l l \rightarrow x=n u l l \vee z=n u l l) \\
D \vDash \forall x y z(R(x, y) \wedge x=\text { null } \rightarrow \text { false }) .
\end{gathered}
$$

For $x=a, y=n u l l$, and $z=b$, the antecedent of the rule is satisfied since $R(a, b) \in D$ and $R(a, n u l l) \in D$ For these values the consequent is not satısfied, because $z=$ null is false. Therefore, Formula (210) does not hold Thus, the database instance $D$ is

\footnotetext{
${ }^{2}$ Without loss of generality we will assume the primary key to be the first $m$ attributes of $R$
} 
inconsistent

\subsection{Query Answering in Databases with Null Values}

In order to answer first-order queries in databases with null values, we will use the null query answering semantics introduced in [11] It extends the semantics of IC satisfaction we just presented

We assume that all the qualifiers in a first-order query are over different var1ables For example, $\forall x P(x, y) \wedge \forall x Q(x)$ can be rewritten as the equivalent query $\forall x P(x, y) \wedge \forall z Q(z)$ The queries may contain the special predicate IsNull, which captures the SQL query with IS NULL and IS NOT NULL expressions in first-order $\log 1 \mathrm{c}$

Example 2.7. Consider a table $P(X, Y)$, and the SQL query $\mathcal{Q}$.

SELECT P.X

FROM $P$

WHERE Y IS NULL

This query $\mathcal{Q}$ can be written in first-order logic as $\exists y(P(x, y) \wedge I s N u l l(y))$.

Definition 12. [11] The set of restricted relevant varıables of a first-order query $\varphi$ are: $\mathcal{V}^{R}(\varphi)=\{x \mid x$ is present at least twıce in $\varphi$, except for the variables in the IsNull predicate $\}$

Example 2.8. For query $\mathcal{Q}: \exists y(P(x, y, z) \wedge Q(y) \wedge I s N u l l(y)), \mathcal{V}^{R}(Q)=\{y\}$, since $y$ is used tw1ce in $\mathcal{Q}$. 
Definition 13. [11] A varıable assignment function $s$, denoted by $s[x \mid a]$, is a function from the set of variables to the underlying database domain $\mathcal{U}$, by setting $s(x)$ to take the value a A term assignment function $\bar{s}$ is defined as follows. (a) If term $t$ is a variable $x$, then $\bar{s}(t)=s(x)$. (b) If term $t$ is a constant $c$ or $n u l l$, then $\bar{s}(t)=c$ Given a formula $\phi, \phi[s]$ denotes the formula obtained from $\phi$ by replacing its free variables by its value according to $s$

Given a variable assignment function $\mathbf{s}$, we check of $D$ satısfies $\phi[\mathbf{s}]$ by assuming that the quantifiers of restricted relevant variables over $(\mathcal{U} \backslash\{n u l l\})$ and of nonrelevant variables over $\mathcal{U}$. Formally.

Definition 14. [11] Let $\phi$ be a first-order formula, and $\mathbf{s}$ be a variable assignment function and $\mathcal{B}=\{\langle\rangle,,=$, false $\}$ We define, by induction on $\phi$, when $D$ satisfies $\phi$ with assignment $\mathbf{s}$ with respect to the null-value semantics, denoted $D \vDash_{N}^{q} \phi[\mathbf{s}]$. Then, $D \vDash_{N}^{q} \phi[\mathbf{s}]$ when $\phi$ is of one of the following forms

$1 t_{1} \diamond t_{n}$ for $\diamond \in\{<,>,=\}, \overline{\mathbf{s}}\left(t_{1}\right) \neq n u l l, \overline{\mathbf{s}}\left(t_{2}\right) \neq n u l l$, and $D \vDash t_{1} \diamond t_{n}$

$2 R\left(t_{1}, \quad, t_{n}\right)$, with $R \in \mathcal{R}$ and $R\left(\overline{\mathbf{s}}\left(t_{1}\right), \quad, \overrightarrow{\mathbf{s}}\left(t_{n}\right)\right) \in D$

$3 \neg \alpha$, and $D \sharp_{N}^{q} \alpha[\overline{\mathbf{s}}]$.

4. $(\alpha \vee \beta)$, and $D \vDash_{N}^{q} \alpha[\overline{\mathbf{s}}]$ or $D \vDash_{N}^{q} \beta[\overline{\mathbf{s}}]$

$5(\alpha \wedge \beta)$, and $D \vDash_{N}^{q} \alpha[\overline{\mathbf{s}}]$ and $D \vDash_{N}^{q} \beta[\tilde{\mathbf{s}}]$.

$6(\forall y)(\alpha)$, and one of the following holds

(a) $y \in \mathcal{V}^{R}(\alpha)$, and for all $a$ in $(\mathcal{U} \backslash\{n u l l\}), D \vDash_{N}^{q} \alpha[\overline{\mathbf{s}}[y \mid a]]$.

(b) $y \notin \mathcal{V}^{R}(\alpha)$, and for all $a \operatorname{in} \mathcal{U}, D \vDash_{N}^{q} \alpha[\overline{\mathbf{s}}[y \mid a]]$

7. $(\exists y)(\alpha)$, and one of the following holds: 
(a) $y \in \mathcal{V}^{R}(\alpha)$, and there exists an $a$ in $(\mathcal{U} \backslash\{n u l l\})$ with $D \vDash_{N}^{q} \alpha[\overline{\mathbf{s}}[y \mid a]]$

(b) $y \notin \mathcal{V}^{R}(\alpha)$, and there exists an $a$ in $\mathcal{U}$ with $D \vDash_{N}^{q} \alpha[\overline{\mathbf{s}}[y \mid a]]$

For all database instance $D, D \neq_{N}^{q}$ false.

Definition 15. [11] A variable assignment $\mathbf{s}$ is null-valld with respect to $\phi$ if for every relevant variable $x$ in $\phi, \mathbf{s}(x) \neq n u l l$.

Definition 16. [11] (a) A tuple $\left(t_{1}, \quad, t_{n}\right)$ with values in $\mathcal{U}$ is an answer from a database $D$ under the null query answering semantics to a FO query $\mathcal{Q}$ with free variables $\left(x_{1}, ., x_{n}\right)$ iff there exists a null-valid assignment $\mathbf{s}$ for $\mathcal{Q}$, such that $\mathbf{s}\left(x_{\imath}\right)=t_{\imath}$, for $\imath=1, \quad, n$, and $D \vDash_{N}^{q} \mathcal{Q}[\mathbf{s}] \quad$ (b) $A n s^{N}(\mathcal{Q}, D)$ denotes the set of answers to $\mathcal{Q}$ obtained from database $D$ under the semantics in (a) (c) If $\mathcal{Q}$ is a sentence (boolean query), the answer under the null query answering semantics is yes Iff $D \vDash_{N}^{q} \mathcal{Q}$ and no otherwise

Example 2.9. Consider $D=\{P(1,1,1), P(2, n u l l, n u l l), P(n u l l, 3,3), Q(n u l l)$, $Q(1), Q(3)\}$, and the query $\mathcal{Q}$. $\exists y z(P(x, y, z) \wedge Q(y) \wedge y>2)$.

For this query, the restricted relevant attribute is $y$, and the only free variable is $x \quad \mathbf{s}_{1}(x)=$ null $1 \mathrm{~s}$ an answer to the query posed to $D$ In fact, the formula $\exists y z(P(x, y, z) \wedge Q(y) \wedge y>2)$ is true in $D$, since there is a value for $y$ which is not null and a value for $z$ that make the formula true Namely, $y=3, z=3$ So, it holds that $D \vDash_{N}^{q} \mathcal{Q}[$ null $]$

An assignment $\mathbf{s}_{\mathbf{2}}(x)=2$ is not an answer to the query The formula $\exists y z(P(x, y, z) \wedge$ $Q(y) \wedge y>2)$ is not true in $D$, since the only value for $y$ that makes the formula true is null. 
Now, $\mathbf{s}_{\mathbf{3}}(x)=1$ is not an answer to the query sine there is not a value for $\mathrm{y}$ which is not null that make $y>2$. In all, $A n s^{N}(\mathcal{Q}, D)=\{n u l l\}$

In [11], it is shown that this null query answering semantics coincides with the classical first-order semantics for queries and databases without null values [12].

On the other hand, conjunctive queries can be syntactically transformed into new FO formulas for which the evaluation can be done by treating null as any other constant.

Definition 17. A query of the form (2.1) can be transformed into a new query $\mathcal{Q}_{N}$ written as $\exists \bar{y}\left(\wedge_{\imath=1}^{n} R_{\imath}\left(\bar{x}_{\imath}, \bar{y}_{\imath}\right) \wedge \varphi \wedge \wedge_{v \in \mathcal{V}^{R}(\mathcal{Q})} v \neq n u l l\right)$, where $\mathcal{V}^{R}(\mathcal{Q})$ are restructed relevant variables (cf Definition 12) In fact, this ensures that relevant variables are evaluated only over values in $(\mathcal{U} \backslash\{n u l l\})$ Then, the new query $\mathcal{Q}_{N}$ can be evaluated treating null as any other constant in $\mathcal{U}$.

Example 2.10. (example 29 contınued) The query $\mathcal{Q}$ in Example 29 can be rewritten as $Q_{N} \cdot \exists y z(P(x, y, z) \wedge Q(y) \wedge y>2 \wedge y \neq n u l l)$

To check if $D \vDash \mathcal{Q}_{N}(1)$, we need to check if $D \vDash \exists y z(P(1, y, z) \wedge Q(y) \wedge y>2 \wedge y \neq$ null) Now, for $y=1$ and $z=1, D \vDash P(1,1,1)$ and $D \vDash Q(1)$, but $y=1$ is less than 2 Therefore (1) is not an answer to the query $\mathcal{Q}_{N}$

(2) is not an answer to the query $\mathcal{Q}_{N}$ Although $D \vDash P(2, n u l l, n u l l)$ and $D \vDash$ $Q(n u l l), y=n u l l$ contradicts with $y \neq n u l l$. Therefore (2) is not an answer.

Now, (3) is an answer to the query $\mathcal{Q}_{N}$, since it holds that $D \vDash(P(n u l l, 3,3) \wedge$ $Q(3) \wedge 3>2 \wedge 3 \neq$ null $)$. 


\subsection{Database Repairs and Consistent Query Answering}

Given a inconsistent database instance $D$ with a set $I C$ of integrity constraints, we can restore consistency by deleting and/or inserting tuples. As a result, a new database instance, so-called reparr, has the same schema as $D$ that satisfies the $I C$ and minimally differs from $D$ under set inclusion In order to formally define the repairs, we need a notion of distance between the database and its reparred version

Definition 18. [5] Let $D, D^{\prime}$ be database instances over the same schema and domain. The distance, $\Delta\left(D, D^{\prime}\right)$, between $D$ and $D^{\prime}$ is the symmetric difference $\Delta\left(D, D^{\prime}\right)=\left(D \backslash D^{\prime}\right) \cup\left(D^{\prime} \backslash D\right)$

Definition 19. [12] Let $D, D^{\prime}, D^{\prime \prime}$ be database instances over the same schema and domain $\mathcal{U}$ It holds that $D^{\prime} \leq_{D} D^{\prime \prime}$ iff

(a) For every atom $P(\bar{a}) \in \Delta\left(D, D^{\prime}\right)$, with $\bar{a} \in(\mathcal{U} \backslash\{n u l l\})$, it holds that $P(\bar{a}) \epsilon$ $\Delta\left(D, D^{\prime \prime}\right)$

(b) For every atom $P(\bar{a}, \overline{n u l l}) \in \Delta\left(D, D^{\prime}\right)$, with $\bar{a} \in(\mathcal{U} \backslash\{n u l l\})$, there exists a $\bar{b} \in \mathcal{U}$, such that $P(\bar{a}, \bar{b}) \in \Delta\left(D, D^{\prime \prime}\right)$ and $P(\bar{a}, \bar{b}) \notin \Delta\left(D, D^{\prime}\right)$

Definition 20. [12] Given a database instance $D$, a set $I C$ of ICs of the form (23), and NNCs of the form (2.7), a repair of $D$ wrt $I C$ is a database instance $D^{\prime}$ over the same schema of $D$, such that.

(a) $D^{\prime} \vDash_{N} I C$,

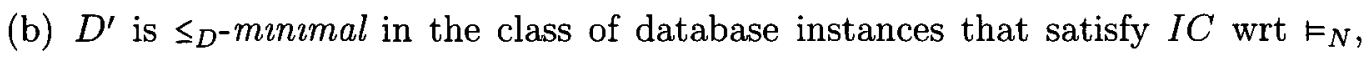
i e. there is no database $D^{\prime \prime}$ in this class with $D^{\prime \prime}<_{D} D^{\prime} . D^{\prime}<_{D} D^{\prime \prime}$ means $D^{\prime} \leq_{D} D^{\prime \prime}$, but not $D^{\prime \prime} \leq_{D} D^{\prime}$ 
The set of repairs of $D$ wrt $I C$ is denoted by $\operatorname{Rep}(D, I C)$

Example 2.11. Consider the database instance $D=\{P(a)\}$, and the integrity constraint IC $\forall x(P(x) \rightarrow \exists z Q(x, z))$ This database $D$ is inconsistent wrt IC Consistency can be restored by deleting $P(a)$ or inserting $Q(a, n u l l)$ So there are two repairs $D_{1}=\{\}$ and $D_{2}=\{P(a), Q(a, n u l l)\}$ Notice that the database instance $D_{3}=\{P(a), Q(a, b)\}$ is consistent wrt IC, but it is not a repair, we have $D_{1} \leq_{D} D_{3}$, therefore $D_{3}$ is not $\leq_{D}$-minımal

A consistent answer to a FO query posed to a possibly inconsistent database $D$ wrt a set $I C$ of $\mathrm{ICs}$ is defined as follows

Definition 21. [12] Given a database $D$ and a set $I C$ of ICs, a ground tuple $\bar{t}$ is a consistent answer to a query $\mathcal{Q}(\bar{x})$ wrt $I C$ in $D$ iff for every $D^{\prime} \in \operatorname{Rep}(D, I C)$, $D^{\prime} \vDash_{N}^{q} \mathcal{Q}[\bar{t}]$ If $\mathcal{Q}$ is a sentence (boolean query), then yes is a consistent answer iff $D^{\prime} \vDash_{N}^{q} \mathcal{Q}$ for every repair $D^{\prime}$ of $D$ Otherwise, the consistent answer is no

In this formulation of CQA we are using a notion $\vDash_{N}^{q}$ of answering first-order queries in databases with null

\subsection{Views}

As indicated above, secret data is characterized as the contents of a view In this research, we will restrict ourselves to a syntactic class of views

Definition 22. A vıew $V_{s}$ is defined by a Datalog rule of the form

$$
V_{s}(\bar{x}) \leftarrow R_{1}\left(\bar{x}_{1}\right) \quad, R_{n}\left(\bar{x}_{n}\right), \varphi
$$

with $R_{\imath} \in \mathcal{R} \bar{x} \subseteq \bigcup_{\imath} \bar{x}_{\imath}$, and $\bar{x}_{\imath}$ is a tuple of variables Formula $\varphi$ is a conjunction of bullt-ins atoms containing variables in $R_{\imath}$ s or domain constants The conjunction in 
the body is denoted by $B\left(V_{s}\right)$.

Thus, in this thesis views are defined by conjunctive queries with built-in predicates $V_{s}[D]$ denotes the extension of view $V_{s}$ when computed on an instance $D$ for $\Sigma$ Sometimes we simply use $V_{s}$ if the instance is understood from the context

Example 2.12. Consider the database instance $D=\{R(a b), R(c, d), S(b, f), S(d, g)$, $S(e, e)\}$, and the view by $V_{s}(x) \leftarrow R(x, y), S(y, z)$ Here, the data in the extension of the view $V_{s}[D]=\{(a),(c)\}$ Sometımes, to emphasize the predicate involved, we write $V_{s}[D]=\left\{V_{s}(a), V_{s}(c)\right\}$.

Our views can be expressed as $L(\Sigma)$-queries More precisely, $B\left(V_{s}\right)$ in (212) can be written as the conjunctive query $\exists \bar{y}\left(R_{1}\left(\vec{x}_{1}\right) \wedge \wedge R_{n}\left(\bar{x}_{n}\right) \wedge \varphi\right)$, with $\bar{x}=\left(\bigcup_{2} \vec{x}_{\imath}\right)-\bar{y}$ 


\section{Chapter 3}

\section{Null Semantics for SQL Queries and ICs}

This research will address the problem of characterizing and obtaining secret answers

to queries, 1 e that do not reveal secret information as specified by secrecy views over incomplete databases that may contain the single null value null, as NULL in SQL relational databases. In order to obtain secret answers in different DBMSs that (possibly only partially) follow the SQL standard that use null values in practice, there are several issues that need to be addressed.

First, we will make use of null to protect secret information contained in secrecy views. The idea is that the extensions of the secrecy views contain only tuples with null or become empty Our view evaluation corresponds to conjunctive query evaluation, which will be based on the notion of the null query answering semantics (cf Chapter 4) We need to show how different DBMSs interpret the null query answering semantics

Second, we can have integrity constraints on the base schema In this case, privacy has the extra condition of satisfying the ICs, because ICs could be violated through the process of updating the database instance with nulls IC satisfaction with null values introduced in $[12,11]$ ( cf Section 21 ) give us a repairıng strategy to restore consistency Therefore, we need to show how different DBMSs interpret the IC satisfaction with null values, since we expect to adopt this semantics to restore consistency

As a result of this chapter, we are able to apply the notion of the null query answering semantıcs in different DBMSs, and we also give some gudelines to adopt 
the semantıcs of IC satisfaction with null values proposed in $[12,11]$ in different DBMSs

In this chapter, we do not consider secrecy instances or secret answers These topics will be addressed in Chapter 4 .

\subsection{Interpretations of Null Values}

A number of research $[24,26,43,41]$ has concentrated on the topic of incomplete databases and null values in relational databases. Incomplete databases in the classic sense that some information is represented using null values. Basically, a null is a place-holder for an attribute whose value can not be represented by an usual constant Formal treatment of nulls usually include many interpretations, however, they can can be classified in two categories [43] These are

(a) Unknown. the attrıbute's value exısts, but it is missing or unknown For example, last name of an employee is missing because every employee has a last name or the salary of employee John is unknown

(b) Inapplıcable the attribute's value is mapplicable or does not exıst, i e. salary for an employee, who just enters the company, is not valıd during the first month. The null for salary is not applicable for at least one month. If an employee has no middle name, a special domain value null could be used to represent the middle name

A formal treatment of null values under "unknown" interpretation was first proposed by Codd [17] and is defined by the usual true and false, and also the additional value unknown, which is used for null In Codd's approach, a comparison expression, such as $X>100$, evaluates to true or false in the usual way if $X$ is not null However, if $X$ is null, then this expression evaluates to unknown The truth tables for the negation, conjunction, and disjunction in three-valued logic (3VL) have also been 
addressed. Table 31 is the truth table for conjunction $(A n d)$ while Table 32 is the true table for disjunction $(O r)$ 3VL for negation $(N o t)$ is defined in the true table shown in Table 33.

Table 3.1: Truth Table for Conjunction

\begin{tabular}{|c|c|c|c|}
\hline AND & True & False & Unknown \\
\hline True & True & False & Unknown \\
False & False & False & False \\
Unknown & Unknown & False & Unknown \\
\hline
\end{tabular}

Table $32 \cdot$ Truth Table for Disjunction

\begin{tabular}{|c|c|c|c|}
\hline OR & True & False & Unknown \\
\hline True & True & True & True \\
False & True & False & Unknown \\
Unknown & True & Unknown & Unknown \\
\hline
\end{tabular}

Table 3 3. Truth Table for Negation

\begin{tabular}{|c|c|}
\hline $\mathrm{P}$ & Not $\mathrm{P}$ \\
\hline True & False \\
False & True \\
Unknown & Unknown \\
\hline
\end{tabular}

3VL was adopted in the SQL 2003 standard (SQL-2003) [1, 2] In general, when a given query is evaluated, only those combinations of tuples that evaluate the logical expression of the query to true are selected as answers. In addition, SQL-2003 suggests the use of $I s$ Null predicate when comparing values in a equality comparison against null For example, a Is Null predicate tests whether one or more columns have null values This predicate returns true if every value of attributes is null, otherwise it returns false

The implemented semantics for query answerıng as specified in the SQL standard follows this three-valued logic, however it has been criticized in the database literature 
for its shaky logical basis. Some tuples will not be returned as answers to some tautological queries [43]. For example if we ask for all the employees with salary > 2000 or salary $\leq 2000$, any employee whose salary is null will not be the answer. That is, if the expression $p$ is unknown, then $\neg p$ is unknown as well, which in turn means $p \vee \neg p$ is unknown instead to true

In addition, both inapplıcable and unknown values are represented by null values The SQL standard and DBMSs won't be able to distınguish them. Logically cleaner versions of null value semantics have been proposed by the research community, i e. four-valued logic was proposed [22] to distinguish applicable and inapplicable data. But they have not been adopted by commercial systems due to the criticisms they have recelved

The semantics introduced in $[12,11]$ provides a partial logical reconstruction in first-order predicate logic of the way nulls are handled in the SQL standard. More precisely, this semantics addresses the notion of IC satısfaction in the presence of null (cf. Section 21 ) and query answerng for a broad classes of queries in databases with/without null values (cf Section 2 2)

In our work, we will adopt this first-order reconstruction of null, because it captures the semantics of the SQL NULL that are relevant for our work on privacy, namely integrity constraint satisfaction and (conjunctive) query answering (cf. Section 4)

\subsection{Null Interpretation in SQL queries}

SQL query answering is the implemented semantics currently used in DBMSs for query answering, which is not an extension of the null query answering semantıcs. In order to check whether the null query answering semantics conforms to the SQL query answering in DBMSs, we basically compare answers of conjunctive queries given by DBMSs with the result of the null query answering semantics. 
A first-order conjunctive query $\mathcal{Q}(\bar{x})$ of the form $(21)$ can be always expressed as a SQL query $\mathcal{Q}^{S Q L}$ as follows

SELECT $x_{1}^{\prime}, . \quad, x_{m}^{\prime}$

FROM $R_{1}, \quad, R_{n}$

WHERE $R_{\imath} \cdot v=R_{\jmath} v$, AND. . AND $R_{l} u=R_{k} u$, AND $\varphi^{S Q L}$

Here, $\bar{x}=\left\{x_{1}, \ldots, x_{m}\right\}, x_{\imath}^{\prime}$ is an attribute associated with variable $x_{\imath}$ in $\mathcal{Q}(\bar{x})$, the conditions in the WHERE clause represent the joins, and $\varphi^{S Q L}$ replaces each variable $x$ in $\varphi$ by its $x^{\prime}$ version

We use the following running example to illustrate how the null query answering semantics is interpreted in DBMSs This is done by creating tables and running querıes in major commercial database management systems. In partıcular, we compare answers obtained by the null query answering semantics with those obtained by SQL query answernng in Oracle 11g, IBM DB2 97 Enterprıze Edition, Microsoft SQL Server 2008 In the following, we will use the abbreviations Oracle, DB2, and SQL Server to refer to above DBMSs

Example 3.1. The employee and department data in Tables 34 and 35 will be used serval times throughout the rest of this chapter. Imagine a company database $D$ storing information for employees and departments as shows in the following two tables. The attributes Dept and Salary in the Employee table may take null values Also the attributes Name in the Dept table are allowed to contain null values

Table 3 4· Employee Table

\begin{tabular}{|c|c|c|c|}
\hline Eno & Name & Dept & Salary \\
\hline 100 & Mike & 1 & 40,000 \\
101 & Mary & 1 & 35,000 \\
102 & Tim & 1 & null \\
103 & John & 2 & 42,000 \\
104 & Anna & null & 45,000 \\
\hline
\end{tabular}


Table $35 \cdot$ Dept Table

\begin{tabular}{|c|c|}
\hline Deptno & Name \\
\hline 1 & HR \\
2 & $I T$ \\
3 & Sales \\
5 & Marketing \\
\hline
\end{tabular}

The following example shows whether SQL query answering coincides with the null query answermng semantics for a conjunctive query that contains joins and built-1ns

Example 3.2. Consider the conjunctive query $\mathcal{Q}: \exists x z w u(\operatorname{Employee}(x, y, z, w) \wedge$ $\operatorname{Dept}(z, u) \wedge u=$ "IT")) It asks for the names of all employees who work at IT department According to the null query answering semantics as presented in Section $22, A n s^{N}(\mathcal{Q}, D)=\{(J o h n)\}$ There are two ways to evaluate this query in DBMSs.

First, this query $\mathcal{Q}$ can be expressed as the following SQL query $\mathcal{Q}^{S Q L}$, which is evaluated in DBMSs as usual

SELECT Employee Name

FROM Dept, Employee

WHERE Dept $=$ Deptno

AND
Dept Name= "IT"

This type of queries is very common in database praxis. If the query $\mathcal{Q}^{S Q L}$ is executed in three DBMSs as usual, all of them return the tuple $\{(J o h n)\}$ Therefore, we can see that the answers to a query of this kind obtained from both the null query answering semantics and SQL DBMSs coincide In fact, they all implement 3VL for the boolean operator $A n d$ as we showed above

Furthermore, query $\mathcal{Q}$ can be transformed into a new SQL query $\mathcal{Q}_{N}^{S Q L}$ which can 
be evaluated treating null as any other constant but making sure that variables in joins do not take the value null. This transformation is done by first rewriting query $\mathcal{Q}$ into a new query $Q_{N}: \exists x z w u(\operatorname{Employee}(x, y, z, w) \wedge \operatorname{Dept}(z, u) \wedge u=" I T " \wedge z \neq$ $n u l l \wedge u \neq n u l l$ ), according to Definition 17 in Chapter 2 Next, this query is expressed in SQL using SQL notation The following is the new transformed SQL query $\mathcal{Q}_{N}^{S Q L}$.

\section{SELECT Employee.Name}

FROM Dept, Employee

WHERE Dept $=$ Deptno AND Dept.Name $=$ 'IT'

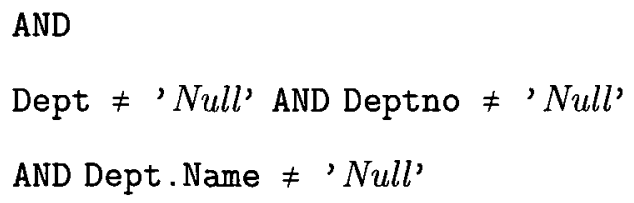

For the purpose of evaluation of query $\mathcal{Q}_{N}^{S Q L}$ on DBMSs treating null as any other constant, we modified the company database $D$ by updating SQL NULL with the constant Null We run this SQL query in our testıng DBMSs, every DBMS returns the tuple $(J o h n)$, as we expected

The following example shows whether SQL query answering coincides with the null query answering semantics for a conjunctive query that is open, and has bult-ins.

Example 3.3. Consider the query $\mathcal{Q} \cdot \exists x z w(\operatorname{Employee}(x, y, z, w) \wedge w<42000)$, that asks for the names of employees whose salary is less than 42,000. According to the null query answering semantics in Section $22, A n s^{N}(\mathcal{Q}, D)=\{($ Mike $),($ Mary $)\}$

Again, query $\mathcal{Q}$ can be written as the following SQL query $\mathcal{Q}^{S Q L}$, and then evaluate $\mathcal{Q}^{S Q L}$ in DBMSs as usual:

SELECT Name

FROM Employee

WHERE Salary $<42000$ 
The set of tuples $\{($ Mike $),($ Mary $)\}$ are returned by running the query $\mathcal{Q}^{S Q L}$ in our three DBMSs Notıce that $(T \imath m)$ is not an answer to the SQL query In this case, the answers given by SQL DBMSs and the null query answering semantics coincides, which means comparison null with any value will be evaluated as unknown in both commercial DBMSs and null querying answering semantics

Similarly, we can transform query $\mathcal{Q}$ into a new SQL query $\mathcal{Q}_{N}^{S Q L}$ for which evaluation can be done by treating null as any other constant

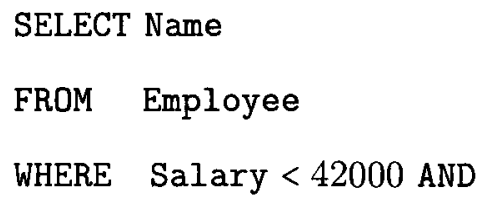

The set of tuples $\{(M \imath k e),(M a r y)\}$ are returned by running the query $\mathcal{Q}_{N}^{S Q L}$ in our three DBMSs

As indicated above, there is a very important predicate Is Null to test certain attributes from taking the value null.

Example 3.4. Consider the following conjunctive query $\mathcal{Q}$ with a $I s$ Null predicate: $\exists x z w(\operatorname{Employee}(x, y, z, w) \wedge \operatorname{IsNull}(z))$, and the corresponding SQL query $\mathcal{Q}^{S Q L}$

\section{SELECT Name}

FROM Employee

WHERE Dept IS Null

For the null query answering semantıcs (cf. Section 22 ), it holds that $\operatorname{Ans}^{N}(\mathcal{Q}, D)=$ $\{($ Anna $)\} \quad$ A tuple containing null for dept will be the shown in the answer of the SQL query $\mathcal{Q}^{S Q L}$ As for this case, (Anna) is the answer in all three DBMSs If we insert tuple $(105$, Smith , ,34,000) into Employee table by using the following SQL 
statement:

INSERT INTO Employee

VALUES (105, 'Smıth',' ',34000)

DB2 and SQL Server return the tuple $(A n n a)$, whle Oracle returns the set of tuples $\{($ Anna $),($ Smith $)\}$ In SQL-2003 and the null query answering semantics, null is not the same as an empty character string, i e. a strung of blank characters. However, Oracle treats a string value with a length of zero as null

In the above examples, we are showing that, the null query answering semantics fully captures the way null values handled on DB2 and SQL Server for first-order conjunctive queries. However, for Oracle, since null means both "unknown" as well as the zero-length empty string, answers to conjunctive queries in Oracle may be different from the null query answering semantıcs Therefore, we need to treat the empty string as null in database in order to capture the semantics of null in Oracle. For example, the database instance $D=\left\{P\left(1,{ }^{\prime} \quad\right.\right.$ ),$\left.Q(n u l l)\right\}$ is equivalent to $D=\{P(1, n u l l), Q($ null $)\}$ in Oracle.

\subsection{IC Satisfaction in Databases with Null Values}

In this section, we start with an overview of the semantics of IC satisfaction in the SQL standard SQL-2003. Then, we compare the semantics of IC satisfaction with null values provided in $[12,11]$ and major commercial database management systems that all follow SQL-2003 Here, we focus on the most important ICs avallable in major commercial databases, such as unıque, primary key, and foreign key constraints. We therefore provide some starting points towards modifying the semantics for satisfaction of constraints in databases with null values in order to unify the semantics currently used in different DBMSs. 


\subsubsection{Semantics of Unique Constraints}

According to SQL-2003, a unique constraint UNIQUE $\left(x_{1}, \quad, x_{n}\right)$ for a relation $R \in \mathcal{R}$ is satısfied iff there are no two rows $t_{1}, t_{2}$ in $R$ such that the values of all their attributes $x_{\imath}$ have the same non-null values More formally [40],

$$
\forall t_{1} t_{2} \in R:\left(\bigwedge_{\imath=1}^{n}\left(t_{1} x_{\imath} \neq n u l l \wedge t_{2} . x_{\imath} \neq n u l l\right) \rightarrow \bigvee_{\imath=1}^{n}\left(t_{1} x_{\imath} \neq t_{2} x_{\imath}\right)\right)
$$

Example 3.5. Consider a database with the table Person, where there is a unique constraint on both Name and Phone together, denoted by UNIQUE (Name, Phone). The following database instance is accepted as a consistent state in SQL-2003

\begin{tabular}{c|c|c|c|}
\hline Person & Name & Phone & Age \\
\hline \multirow{1}{*}{ Mary } & $000-1000$ & 10 \\
& Mary & $000-1001$ & 20 \\
null & $000-1000$ & 30 \\
\hline
\end{tabular}

This is because null in Name attribute is not relevant to check IC satısfaction More precisely, for these values in Name or Phone which are null make the antecedent of Formula (3.1) false Therefore the whole formula is true

Example 3.6. To simplify the presentation, we further assume that the name of department has to be unique The table of definition in Example 31 was extended by adding the following unique constraint.

ALTER TABLE Dept

ADD CONSTRAINT name_Unıque UNIQUE (Name) 
As discussed in Section 2.12 , the name_Unıque can be written as a set of rules according to the semantics proposed in [11].

$$
\begin{aligned}
& \forall x y z(\operatorname{Dept}(y, x) \wedge \operatorname{Dept}(z, x) \wedge I s N u l l(y) \rightarrow I s N u l l(z)) \\
& \forall x y z(\operatorname{Dept}(y, x) \wedge \operatorname{Dept}(z, x) \rightarrow y=z)
\end{aligned}
$$

In order to check if the company database $D$ satisfies the name_Un $q$ que, by Definition 8, we need to check the following formulas:

$$
\begin{aligned}
& D \vDash \forall x y z(\operatorname{Dept}(y, x) \wedge \operatorname{Dept}(z, x) \wedge y=n u l l \rightarrow x=n u l l \vee z=n u l l) . \\
& D \vDash \forall x y z(\operatorname{Dept}(y, x) \wedge \operatorname{Dept}(z, x) \rightarrow x=n u l l \vee y=n u l l \vee z=n u l l \vee y=z)
\end{aligned}
$$

DB2 disallows the unique constraint name_Unıque, since DB2 does not support unique constraints on columns that contain null values, whle both Oracle and SQL Server allow it The following insertıon is accepted by Oracle, SQL server, and the semantics in $[11]$

\section{INSERT INTO Dept}

$\operatorname{VALUES}(6, \operatorname{NULL})$

However, if we try to insert another null value into Name in the Dept table, the insertion will be rejected by SQL Server due to violation of the unique constraint name_Unıque. Basıcally, in SQL Server, if a unique constraint is defined upon a column containing null, only one null value will be allowed in that column, while in Oracle and [11] a unique constraint allows more than one null values Therefore, the semantıcs of a unıque constraint defined in Oracle is less restrictive More formally, $\operatorname{UNIQUE}\left(x_{1}, \ldots, x_{n}\right)$ for a relation $R$ holds in DB2 iff the following holds [40]:

$$
\forall t \in R:\left(\bigwedge_{\imath=1}^{n} t . x_{\imath} \neq n u l l\right) \wedge \forall t_{1} t_{2} \in R:\left(\bigvee_{\imath=1}^{n} t_{1} x_{\imath} \neq t_{2} x_{\imath}\right)
$$

$\operatorname{UNIQUE}\left(x_{1}, \ldots, x_{n}\right)$ for a relation $R$ holds in SQL Server iff the following holds

$$
\forall t_{1} t_{2} \in R:\left(\bigvee_{\imath=1}^{n} t_{1} . x_{\imath} \neq t_{2} x_{\imath}\right)^{1}
$$

\footnotetext{
${ }^{1}$ Here, null is treated as a database constant value
} 
$\operatorname{UNIQUE}\left(x_{1}, \ldots, x_{n}\right)$ for a relation $R$ holds in Oracle iff the following holds [40]:

$$
\forall t_{1} t_{2} \in R:\left(\bigvee_{\imath=1}^{n}\left(t_{1} x_{\imath} \neq n u l l \vee t_{2} . x_{\imath} \neq n u l l\right) \rightarrow \bigvee_{\imath=1}^{n} t_{1} x_{\imath} \neq t_{2} . x_{\imath}\right)
$$

\subsubsection{Semantics of Primary Key Constraints}

In SQL-2003, a primary key constraint is a combination of a unique constraint and one or more not null constraints. A primary key constraint PRIMARY KEY $\left(\mathbf{x}_{1}, \ldots, \mathbf{x}_{\mathrm{n}}\right)$ for a relation $R \in \mathcal{R}$ is satısfied iff the following formula holds [40]

$$
\forall t \in R:\left(\bigwedge_{\imath=1}^{n} t . x_{\imath} \neq n u l l\right) \wedge \forall t_{1} t_{2} \in R:\left(\bigvee_{\imath=1}^{n} t_{1} . x_{\imath} \neq t_{2} x_{\imath}\right)
$$

In DB2, SQL Server, Oracle, and [11] the definition of a primary key implicitly defines not null constraints on the corresponding uniqueness columns which coincides with the semantics of a primary key proposed in SQL-2003, as shown in the following example

Example 3.7. (example 36 contınued) The table of definition in Example 36 was extended by adding the following primary key.

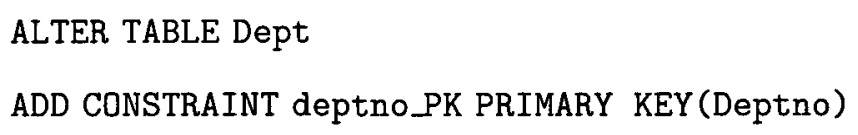

According to [11], the primary key can be written as $\forall x y z(\operatorname{Dept}(x, y) \wedge \operatorname{Dept}(x, z) \rightarrow$ $y=z), \forall x y z(\operatorname{Dept}(x, y) \wedge \operatorname{Dept}(x, z) \wedge \operatorname{IsNull}(y) \rightarrow \operatorname{IsNull}(z)$, and the NNC $\forall x y z(\operatorname{Dept}$ $(x, y) \wedge \operatorname{IsNull}(x) \rightarrow$ false $)$

In order to check if the company database $D$ satisfies the deptno_PK, we need to check the following formulas (cf. Section 2.12 )

$$
\begin{aligned}
& D \vDash \forall x y z(\operatorname{Dept}(x, y) \wedge \operatorname{Dept}(x, z) \wedge y=n u l l \rightarrow x=n u l l \vee z=n u l l) . \\
& D \vDash \forall x y z(\operatorname{Dept}(x, y) \wedge \operatorname{Dept}(x, z) \rightarrow x=n u l l \vee y=n u l l \vee z=n u l l \vee y=z) . \\
& D \vDash \forall x y z(\operatorname{Dept}(x, y) \wedge x=\text { null } \rightarrow \text { false }) .
\end{aligned}
$$


The following insertion is rejected due to a violation of the primary key deptno_PK in DB2, Oracle and SQL Server

INSERT INTO Dept

VALUES (NULL, 'Consultıng')

The deptno_PK will be volate the primary key semantics proposed in [11] if there is tuple (null, consultıng) in the Dept table. This is because the tuple Dept (null, consulting) will not be satisfied Formula (3.10)

\subsubsection{Semantics of Foreign Key Constraints}

Referential constraints permit the comparison of values from different columns of one or more relations A referential constraint (or an inclusion dependency), denoted by $R[X] \subseteq S[Y]$ (cf Sectıon 21), is satısfied, if every value of $X$ in $R$ that refers to a value of the corresponding attribute in $Y$ in the relation $S^{2}$ Here, $R$ is the referencing table and $S$ is the referenced table In the case of referential constraints, SQL-2003 suggests three different semantıcs, namely simple, partıal, and full, respectively

If it is simple-match semantics [2] for each row $t_{1}$ of the referencing table $R$, either at least one of the values of the referencing columns in $R$ shall be a null value, or the value of each referencing column in $R$ shall be equal to the value of the corresponding referenced column in some row of the referenced table $S$

If partıal-match semantics [2] is specified, for each row $t_{1}$ of the referencing table $R$, there shall be some row $t_{2}$ of the referenced table $S$ such that the value of each referencing column in $t_{1}$ is either $n u l l$ or is equal to the value of the corresponding referenced column in $t_{2}$.

If full-match semantics [2] is specified, for each row $t_{1}$ of the referencing table $R$, either the value of every referencing column shall be a null value, or the value of every referencing column in $t_{1}$ shall not be null and there shall be some row $t_{2}$ of the

\footnotetext{
${ }^{2}$ Referential constraints could be defined on the same table
} 
referenced table such that the value of each referencing column in $t_{1}$ is equal to the value of the corresponding referenced column in $t_{2}$

Example 3.8. Consider a referenial constraint $\psi: R(X, Y) \subseteq S(X, Y)$ and the database $D=\{R($ null,a), S(a,a) $\}$. (a) $D$ satisfies $\psi$ wrt simple match, since there is a null value in the referencing table $R$ (b) $D$ does not satisfy $\psi$ wrt partial match, since there is no tuple $R(a, a)$ or $R($ null, null) in $R$ (c) $D$ does not satisfy $\psi$ wrt full match, since any of null values can not be in the referencing table $R$

Foreıgn key is a special case of a referential constraint It requires the primary key on the referenced table

Example 3.9. In order to check the semantics of foreign keys, our company database was extended by assuming the a employee's department must be a department of the company The table definition of Example 37 was extended by

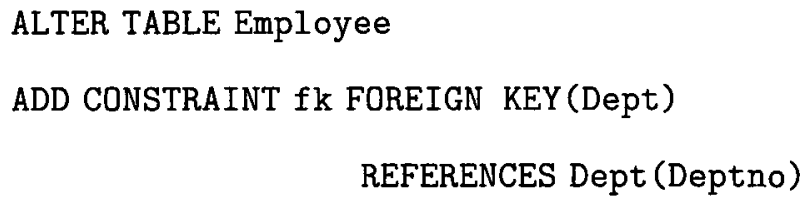

In [11], the $\mathrm{fk}$ can be written as $\psi: \forall x y z w(\operatorname{Employee}(x, y, z, w) \rightarrow \exists u \operatorname{Dept}(z, u))$ Variable $z$ is relevant to check the constraint, therefore the set of relevant attributes is $\mathcal{A}(\psi)=\{$ Employee[3],Dept [1] $\}$ To check the fk, by Definition 8, we need to check $D \vDash \forall z\left(\operatorname{Employee}^{\mathcal{A}}(z) \rightarrow z=n u l l \vee D e p t^{\mathcal{A}}(z)\right)$, where Employee $\operatorname{Ai}^{\mathcal{A}}$ and $D e p t^{\mathcal{A}}$ are the predicates projected onto the relevant attributes In this example, the company database $D$ satisfies the foreign key constraint $\mathrm{fk}$.

A possible way to check satisfaction of an integrity constraint in DBMSs is by means of violation view $[23,38]$. If the integrity constraint is satisfied, then violation 
view is empty, otherwise it contains the set of tuples that violates the constraint.

Example 3.10. (example 39 continued) For the foreign key in Example 3.9, the following query $\mathcal{Q}$ defines the violation view for databases with null values, and is used to check the satisfaction of $\mathrm{fk}$.

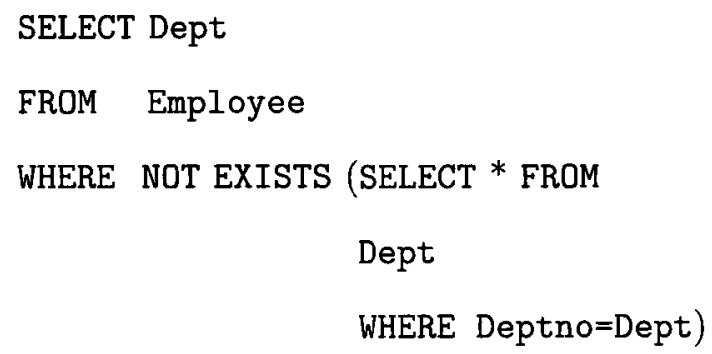

AND Dept IS NOT NULL

By running this query $\mathcal{Q} 1$ three DBMSs, result of query $\mathcal{Q}$ is empty in every DBMS Therefore, in all DBMSs, null values in referencing columns do not violate foreign key constraints

In this section, we focused on unvque, primary, and foreign key constraints satisfaction in the presence of null supported by DB2, Oracle, SQL Server, and the semantics proposed [11] We give the results of observations from the above examples:

- Unıque key. This type of integrity constraint is supported by all testing systems and the semantics in [11]. Notice that Oracle and the semantics in [11] support multiple null values in the unique key column whereas SQL Server allows only one null value in the unique key column. In SQL Server, this is contradiction of null comparison of $3 \mathrm{VL}$ in SQL-2003, this is because, null $=$ null returns true instead of unknown

- Primary key All testing systems and the semantıcs in [11] support the primary key constraint which coincides with semantics of the primary key proposed in 
SQL-2003

- Forergn key All testing systems and the semantics in [11] implement only the simple-match semantıcs for foreign key constraints suggested in SQL-2003, which allows to contain null values in referencing columns They do not support the partial-match and full-match semantics suggested in SQL-2003 [12, 40].

In [11], they present a repair semantics to restore consistency in the context of incomplete databases, which is based on the notion of IC satisfaction proposed in [11]. In our privacy work, we will make use of this semantics to obtain consistent and secret answers on the top of different DBMSs, therefore this semantics should be compatible with the way null values are treated in DBMSs Above investigations give us some starting points on modifying this semantics in order to unify the semantics currently used in different DBMSs 


\section{Chapter 4}

\section{A Semantics for Privacy with Uncertain Data}

The privacy problem, as illustrated in Chapter 1, will be solved by performing a minimal set of virtual changes on the database instances, which captures the ideas of (a) Having a class of virtual secrecy instances for a database that protects secret data as defined by the secrecy views, (b) Minimizing the distance between a secrecy instance and the given database instance, and this minımization takes into account the occurrence of null values (c) Computing answers to a query that do not reveal any secret information

In this chapter, we start in Section 41 with the semantics of secrecy in the presence of $n u l l$, and we also characterize a class of secrecy instances. Then we introduce the precise definition of secret answers to first-order conjunctive queries in Section 42 . In this research we restrict ourselves to conjunctive queries. An extension of our semantics for queries containing negation is left for future work

\subsection{Secrecy Instances of Incomplete Databases}

In this work we will make use of null to protect secret information As described in Section 32 , the semantics of null will (essentially) correspond to the way nulls are handled by DBMSs that follow the SQL standard. The idea is that the extensions of the secrecy views will contain only tuples with null or will become empty Our view evaluation problem corresponds to conjunctive query evaluation, which will be based on the notion of the null query answernng introduced in Section 22 
Example 4.1. (example 29 continued) Consider the following instance $D$ :

\begin{tabular}{c|c|c|c|c|c|}
\hline$P$ & $X$ & $Y$ & $Z$ \\
\hline & 1 & 1 & 1 \\
2 & null & null \\
& null & 3 & 3 & & \\
\cline { 2 - 5 } & & & \\
& &
\end{tabular}

and the secrecy view $V_{s}(x) \leftarrow P(x, y, z), Q(y), y>2$. This secrecy view can be expressed as the first-order query

$$
\mathcal{Q}^{V_{s}}(x): \exists y z(P(x, y, z) \wedge Q(y) \wedge y>2),
$$

which is the same query as in Example 29.

Under the semantics of secrecy in the presence of null, we expect that the extensions of the secrecy views contain tuples only with null or become empty This implies that the values in attribute $X$ associated with variable $x$ in $\mathcal{Q}^{V_{s}}$ are $n u l l$, or the values In $Y$ associated with variable $y$ in $\mathcal{Q}^{V_{s}}$ are null, or the negation of the comparison is true These three cases correspond to the three assignments in Example 2.9 Notice that the attributes $X$ and $Y$ play an important role for the semantics of secrecy $\square$

The above example shows that the occurrence of null $\mathrm{m}$ some attributes are crucial to gain the view extensions In the following, we introduce the notion of null-special attributes (NSA) for a secrecy view to capture the position null in attributes, $1 \mathrm{e}$. those that associate with variables involved in joins, bult-ins, and the head of the view. This is because the position null in joins and bult-ins will make the view extensions empty, whlle the position null in the head will make the view extensions contain tuples only with null

Definition 23. For a Datalog rule of the form (212) and a term $t$ (i e a variable or constant), let $\operatorname{pos}^{R}\left(V_{s}, t\right)$ be the set of positions in predicate $R \in \mathcal{R}$ where $t$ appears $\square$ 
Example 4.2. Consider a secrecy view: $V_{s}(x, z) \leftarrow P(x, y), Q(y, z)$. For a variable $y, \operatorname{pos}^{R}\left(V_{s}, y\right)=\{2,1\}$, which means that $y$ appears in the second position in $P$ and in the first position in $Q$. The notion of $\operatorname{pos}^{R}$ in Definition 23 is essentrally the same as the one presented in Chapter 2 Defintion 6

Definition 24. The set of combination attributes for a secrecy view $V_{s}$ in the form of (212) is $\mathcal{C}\left(V_{s}\right)=\{R[\imath] \mid x$ is a variable appearing at least twice in the body of (2 12), and $\left.\imath \in \operatorname{pos}^{R}\left(V_{s}, x\right)\right\}$

Here, $R[\imath]$ denotes a position (or the correspondent attribute) in relation $R$ In fact, combination attributes of a secrecy view $V_{s}$ are those involved in joins or bult-in predicates, and they also correspond to the restructed relevant varıables (cf Definition 12) of the associated query $\mathcal{Q}^{V_{s}}$

Example 4.3. (example 42 continued) The secrecy view in Example 42 can be expressed as the first-order query

$$
\exists y(P(x, y) \wedge Q(y, z))
$$

Since $y$ appears twice in the body of the secrecy view, by Definition $24, \mathcal{C}\left(V_{s}\right)=$ $\{P[2], Q[1]\} \quad$ According to Definition 12, the restricted relevant variable of query (41) is $y$ We can see that the restricted relevant variable $y$ corresponds to combinatron attributes of the secrecy view

Definition 25. The set of secrecy attributes for a secrecy view $V_{s}$ as in (212) is $\mathcal{S}\left(V_{s}\right)=\left\{R[\imath] \mid x\right.$ is a variable in the head of $(212)$, and $\left.\imath \in \operatorname{pos}^{R}\left(V_{s}, x\right)\right\}$ 
Here, secrecy attributes of a secrecy view $V_{s}$ are those appearing in the head of $V_{s}$ 's definition. They correspond to the free variables in the associated query $\mathcal{Q}^{V_{s}}$

Example 4.4. (example 43 continued) Since $x$ and $z$ appear in the head of the secrecy view in Example 4.2, by Definition 25, $\mathcal{S}\left(V_{s}\right)=\{P[1], Q[2]\}$. The free varrables of query (4.1) are $x$ and $z$, which correspond to secrecy attributes of the associated secrecy view

Definition 26. The set of null-specıal attrıbutes for a secrecy view $V_{s}$ are those (associated to positions) in the set $\mathcal{N}\left(\mathcal{V}^{s}\right)=\mathcal{C}\left(V_{s}\right) \cup \mathcal{S}\left(V_{s}\right)$

Example 4.5. (example 41 continued) Consider the same secrecy view in Example $41 V_{s}(x) \leftarrow P(x y, z), Q(y) . y>2$ Since $y$ appears three times in the body, by Definition 24, $\mathcal{C}\left(V_{s}\right)=\{R[2], S[1]\} \quad$ Since $x$ appears in the head, $\mathcal{S}\left(V_{s}\right)=\{R[1]\}$ Therefore, $\mathcal{N}\left(V_{s}\right)=\{R[1], R[2], S[1]\}$. The values in attribute $Z$ are not crucial for gaining the view extensions, no matter whether they are null or not. This makes sense, because we only join tuples via attribute $Y$, and making sure that values in attribute $Y$ are greater than 2 And then, we project values on attribute $X$

Definition 27. A database instance is admıssıble for a set $\mathcal{V}^{s}$ of secrecy views of the form (2.12), denoted $D \in \operatorname{Adm\imath ss}\left(\mathcal{V}^{s}\right)$, if under the $\vDash_{N}^{q}$ semantics, each $V_{s}[D]$ is empty or in all its tuples only null appears

From the example above, we can see that, given a database instance $D$ and a secrecy view $V_{s}$ of the form (212), $D$ is admissible, when one of the following three cases occurs (a) There is a null in any of the combination attributes, (b) Values in 
the secrecy attributes are all $n u l l$, (c) The negation of the built-ins is true.

Before making this claim formal, let us recall that, given a set of attrıbutes $\mathcal{A}$, and a predicate $R \in \mathcal{R}$, we denote by $R^{\mathcal{A}}$ the predicate $R$ projected onto the attributes in $\mathcal{A} D^{\mathcal{A}}$ denotes the database $D$ with all its database atoms projected onto the attributes in $\mathcal{A}$ (cf Definitıon 7$)$

Proposition 1. Let $\mathcal{V}^{s}$ be a set of secrecy views $V_{s}$ of the form (2.12). Let $\mathcal{Q}^{V_{s}}$ be their expressions as conjunctive queries of the form $\exists \bar{y}\left(\wedge_{\imath=1}^{n} R_{\imath}\left(\bar{x}_{\imath}\right) \wedge \varphi\right)$ For an instance $D, D \in \operatorname{Adm\imath ss}\left(\mathcal{V}^{s}\right)$ iff $D^{\mathcal{N}\left(V_{s}\right)} \vDash \mathcal{Q}_{N}^{V_{s}}$, for each $V_{s} \in \mathcal{V}^{s}$, where $\mathcal{Q}_{N}^{V_{s}}$ is

$$
\forall \bar{x}\left(\bigwedge_{\imath=1}^{n} R_{\imath}^{\mathcal{N}\left(V_{s}\right)}\left(\bar{x}_{\imath}\right) \rightarrow \underset{v_{\jmath} \in\left(\mathcal{C}\left(V_{s}\right) \cap \bar{x}\right)}{\bigvee} v_{\jmath}=n u l l \vee \bigwedge_{u_{\jmath} \in\left(\mathcal{S}\left(V_{s}\right) \cap \bar{x}\right)} u_{\jmath}=n u l l \vee \neg \varphi\right)
$$

where $\bar{x}=\bigcup_{\imath=1}^{n} \bar{x}_{\imath}$ Here, $D^{\mathcal{N}\left(V_{s}\right)} \vDash \mathcal{Q}_{N}^{V_{s}}$ refers to classical first-order satisfactıon and null is treated as any other constant

Example 4.6. (example 4.5 continued) According to the above definition, in order to check whether the database instance $D$ is admissible, the following must hold

$$
D^{\mathcal{N}\left(V_{s}\right)} \vDash \forall x y\left(R^{\mathcal{N}\left(V_{s}\right)}(x, y) \wedge S^{\mathcal{N}\left(V_{s}\right)}(y) \rightarrow y=n u l l \vee x=n u l l \vee y<=2\right),
$$

with $D^{\mathcal{N}\left(V_{s}\right)}$ given below. When checking this, null is treated as any other constant in $\mathcal{U}$

\begin{tabular}{c|c|c|}
\hline$R^{\mathcal{N}\left(V_{s}\right)}$ & $X$ & $Y$ \\
\hline \multirow{1}{*}{ null } & 3 \\
\cline { 2 - 3 } & 1 & 1 \\
& &
\end{tabular}

\begin{tabular}{c|c|}
\hline$S^{\mathcal{N}\left(V_{s}\right)}$ & $Y$ \\
\hline & null \\
1 \\
3 \\
\cline { 2 - 2 }
\end{tabular}

For $x=1, y=1$, the antecedent of the implication is satisfied since $R^{\mathcal{N}\left(V_{s}\right)}(1,1) \epsilon$ $D^{\mathcal{N}}, S^{\mathcal{N}\left(V_{s}\right)}(1) \in D^{\mathcal{N}}$. For these values, the consequent is also satisfied.

because $y=1<2$ For $x=2, y=n u l l$, the consequent is satisfied since $y$ is null For $x=n u l l, y=3$, the antecedent is satisfied since $R^{\mathcal{N}\left(V_{s}\right)}(n u l l, 3) \in D^{\mathcal{N}}, S^{\mathcal{N}\left(V_{s}\right)}(3) \in D^{\mathcal{N}}$ For these values, the consequent is also satisfied, because $n u l l=$ null is true In this 
example, $D \vDash_{N} \mathcal{Q}^{V_{s}}$. Therefore, the given instance $D$ is admissible.

Proof of Proposition 1: First we proof that if $D \in \operatorname{Adm\imath ss}\left(\mathcal{V}^{s}\right)$, then each $V_{s}[D]$ is empty or in all its tuples only null appears under the $\vDash_{N}^{q}$ semantics. Here, $V_{s}$ is of

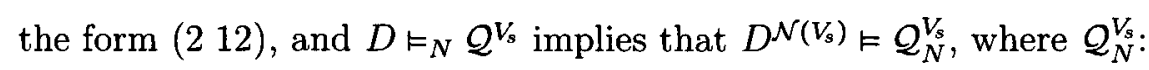

$$
\forall \bar{x}\left(\bigwedge_{\imath=1}^{n} R_{\imath}^{\mathcal{N}\left(V_{s}\right)}\left(\bar{x}_{\imath}\right) \rightarrow \bigvee_{v_{j} \in\left(\mathcal{C}\left(V_{s}\right) \cap \bar{x}\right)} v_{\jmath}=n u l l \vee \bigwedge_{u_{\jmath} \in\left(\mathcal{S}\left(V_{s}\right) \cap \bar{x}\right)} u_{\jmath}=n u l l \vee \neg \varphi\right)
$$

By contradiction, we assume that there exists an assignment $\bar{c}=\left(c_{1}, \quad, c_{n}\right)$ for free variables $\bar{x}=\left(x_{1}, ., x_{n}\right)$ in $\mathcal{Q}^{V_{s} 1}$, such that the following three statements hold.

1 If $x_{\imath} \in \mathcal{V}^{R}\left(\mathcal{Q}^{V_{s}}\right)$, then $c_{\imath} \in(\mathcal{U} \backslash\{n u l l\})$, otherwise, $c_{\imath} \in \mathcal{U}$

2 There exists at least one $\imath \in(1, . \quad, n)$, such that $c_{\imath} \neq n u l l$

$3 \quad D \vDash_{N}^{q} \mathcal{Q}^{V_{s}}[\bar{s}[\bar{x} \mid \bar{c}]]$

$D \vDash_{N}^{q} \mathcal{Q}^{V_{s}}[\bar{s}[\bar{x} \mid \bar{c}]]$ implies that $D \vDash_{N}^{q} \exists \bar{z} \bar{w}\left(\bigwedge_{\imath=1}^{n} R_{\imath}\left(\bar{c}_{\imath}, \bar{z}_{\imath}, \bar{w}_{\imath}\right) \wedge \varphi[\bar{s}[\bar{x} \mid \bar{c}]]\right)$, where $\bar{z}=$ $\bigcup_{\imath=1}^{n} \bar{z}_{\imath}, \bar{w}=\bigcup_{\imath=1}^{n} \bar{w}_{\imath}$, and $\bar{z} \cup \bar{w}=\bar{y}$ Here, variables in $\bar{z}$ are restricted relevant varıables in $\mathcal{Q}^{V_{s}}$, while no variable in $\bar{w}$ is relevant By Definition 14, for all $\bar{a} \in(\mathcal{U} \backslash\{$ null $\})$ and $\bar{d} \in \mathcal{U}$, it holds that $D \vDash_{N}^{q} \wedge_{\imath=1}^{n} R_{\imath}\left(\bar{c}_{\imath}, \bar{a}_{\imath}, \bar{d}_{\imath}\right) \wedge \varphi$. This indicates that $D \notin \underset{v_{\jmath} \in \mathcal{V}^{R}\left(\mathcal{Q}^{\left.v_{s}\right)}\right.}{\vee} v_{\jmath}=$ null, and $D \vDash \varphi$ Therefore, $D^{\mathcal{N}\left(V_{s}\right)} \neq \underset{v_{j} \in\left(\mathcal{C}\left(V_{s}\right) \cap \bar{x}\right)}{\vee} v_{\jmath}=$ null since all free variables $\bar{x}$ in $\mathcal{V}^{R}\left(\mathcal{Q}^{V_{s}}\right)$ are associated with secrecy attributes in $V_{s}$, and we have that $c_{\imath} \neq n u l l$ for at least one $\imath \in(1, \ldots, n)$ Therefore, it holds that $D^{\mathcal{N}\left(V_{s}\right)} \not \bigwedge_{u_{\jmath} \in\left(S\left(V_{s}\right) \cap \bar{x}\right)} u_{\jmath}=$ null Thus, we end up a contradiction with $D \vDash_{N} \mathcal{Q}^{V_{s}}$

Now, Let us prove that under the $\vDash_{N}^{q}$ semantics, $V_{s}[D]$ is empty or in all its tuples only null appears, then $D \vDash_{N} \mathcal{Q}^{V_{s}}$

1 First, we consider that $V_{s}[D]$ are tuples containing only null values By Definition 14, there exists an assignment $\bar{c}=\left(c_{1}, \quad, c_{\imath}\right)$, where $\Lambda_{\imath=1}^{n} c_{\imath}=$ null for free

\footnotetext{
${ }^{1}$ As indicated in Section $24, V_{s}$ can be can be written as the conjunctive query $\exists \bar{y}\left(R_{1}\left(\bar{x}_{1}\right) \wedge\right.$ $\left.\wedge R_{n}\left(\bar{x}_{n}\right) \wedge \varphi\right)$, with $\bar{x}=\left(\cup_{2} \bar{x}_{\imath}\right)-\bar{y}$
} 
variables $\bar{x}=\left(x_{1}, ., x_{n}\right)$ in $\mathcal{Q}^{V_{s}}$ Since all free variables $\bar{x}$ are associated with secrecy attributes in $V_{s}$, it holds that $D^{\mathcal{N}\left(V_{s}\right)} \vDash \bigwedge_{u_{j} \in\left(S\left(V_{s}\right) \cap \bar{x}\right)} u_{j}=$ null which makes $D \vDash_{N} \mathcal{Q}^{V_{s}}$ true.

2. Now, $V_{s}[D]$ is the empty set. By definition 15 , there is no null-valid assignment with respect to $\mathcal{Q}^{V_{s}}$, such that $v_{\jmath} \neq$ null for every $v_{\jmath} \in \mathcal{Q}^{V_{s}}$. This means that there is at least one restricted relevant attribute $v_{\jmath}$, such that $v_{\jmath}=n u l l$. Therefore, $D^{\mathcal{N}\left(V_{s}\right)}=\underset{v_{\jmath} \in\left(C\left(V_{s}\right) \cap \bar{x}\right)}{\bigvee} v_{\jmath}=$ null which makes $D \vDash_{N} \mathcal{Q}^{V_{s}}$ true.

Virtual updates based on null will be used to obtain admissible instances starting from a given instance $D$. A secrecy instance for $D$ will be admissible and will also minimally differ from $D$ The latter condition requires a suitable comparison of database instances As expected, this comparıson will consider the presence of null in the tuples of those instances

Definition 28. [32] (less or equal informative constants) We define a partial order in database domain $\mathcal{U}$, denoted by $ᄃ$, as follows $c \sqsubseteq d$ iff $c=d$ or $c=n u l l$, where $c, d \in \mathcal{U} \square$

This partial order implies that constant $c$ provide less or equal information than a constant $d$ in database Now, we extend $\subseteq$ as a partial order on tuples

Definition 29. [32] (less or equal informative tuples) Let $t_{1}=\left(c_{1}, \quad, c_{n}\right)$ and $t_{2}=\left(d_{1}, \ldots, d_{n}\right)$ be finite sequences of constants. $t_{1}$ is less or equal informative than $t_{2}$, denoted by $t_{1} \subseteq t_{2}$ iff $c_{2} \subseteq d_{\imath}$ for $\forall \imath \in\{1, \quad, n\}$. Also, $t_{1}$ is less informative than $t_{2}$, denoted by $t_{1} \sqsubset t_{2}$ iff $t_{1} \sqsubseteq t_{2}$ and $t_{1} \neq t_{2}$.

For example, tuple $(a, n u l l)$ provides less information than tuple $(a, b)$ Then $(a, n u l l)$ 吊 $(a, b)$ holds. 
Definition 30. Let $D, D^{\prime}, D^{\prime \prime}$ be database instances over the same schema $\Sigma$ and domain $\mathcal{U}$. It holds that $D^{\prime} \leq_{D} D^{\prime \prime}$ iff for every tuple $t=R(\bar{a}) \in D^{\prime}$, there is a unique tuple $t^{\prime}$ such that $t^{\prime} \in D^{\prime \prime}$ and $t^{\prime} \subseteq t . D^{\prime}<_{D} D^{\prime \prime}$ means $D^{\prime} \leq_{D} D^{\prime \prime}$, but not $D^{\prime \prime} \leq_{D} D^{\prime} \square$

Here, $D^{\prime}$ and $D^{\prime \prime}$ are usually instances obtained by possible value updates on $D$ using null If $D^{\prime}<_{D} D^{\prime \prime}$, we say that $D^{\prime}$ is closer to $D$ than $D^{\prime \prime}$. We may assume that tuples have identifiers, so the unque tuple $t^{\prime}$ above will be denoted by $\mu(t)$ Therefore, it is possible to correlate the original tuples with those in the null-based updated instance. The tuples $t, t^{\prime}$ in Definition 30 will be then correlated tuples.

Definition 31. Given a database instance $D$ and a set $\mathcal{V}^{s}$ of views $V_{s}$ of the form (2 12), a secrecy instance $D_{s}$ for $D$ wrt $\mathcal{V}^{s}$ is an instance over the same schema, such that (a) $D_{s} \in \operatorname{Adm\imath ss}\left(\mathcal{V}^{s}\right)$, and (b) $D_{s}$ is $\leq_{D^{-}}$minımal in the class of database instances that satisfy (a) and share the schema with $D, 1$ e there is no instance $D_{s}^{\prime}$ in that class with $D_{s}^{\prime}<_{D} D_{s} \operatorname{Sec}\left(D, \mathcal{V}^{s}\right)$ denotes the set of all the secrecy instances for $D$ wrt $\mathcal{V}^{s}$

Example 4.7. Given the instance $D=\{P(1,2), R(2,1)\}$, and the secrecy vrew $V_{s}(x, z) \leftarrow P(x, y), R(y, z), y<3$, consider the alternatıve updated instances $D_{\imath}$.

Notice that, all updated instances $D_{1}, D_{2}$, $D_{3}$ and $D_{4}$ satisfy the first condition, of admissibility, of secrecy instance in Definition 31 , that $1 \mathbf{s}^{\circ}$

\begin{tabular}{|c|c|}
\hline$\imath$ & $D_{\imath}$ \\
\hline 1 & $\{P($ null,2),R(2,null $)\}$ \\
2 & $\{P(1, n u l l), R(2,1)\}$ \\
3 & $\{P(1,2), R($ null, 1$)\}$ \\
4 & $\{P(1, n u l l), R($ null, 1$)\}$ \\
\hline
\end{tabular}

$D_{\imath}^{\mathcal{N}\left(V_{s}\right)} \vDash \forall x y\left(P^{\mathcal{N}\left(V_{s}\right)}(x, y) \wedge R^{\mathcal{N}\left(V_{s}\right)}(y, z) \rightarrow y=n u l l \vee x=n u l l \wedge z=n u l l \vee y \geq 3\right)$, with $D_{\imath}^{\mathcal{N}\left(V_{s}\right)}=\left\{P^{\mathcal{N}\left(V_{s}\right)}(1,2), R^{\mathcal{N}\left(V_{s}\right)}(2,1)\right\}$ However, $D_{4}$ is not a secrecy instance, because $P(1, n u l l) \subset P(1,2)$; and then, $D_{3}<_{D} D 4$ Thus, $D_{1}, D_{2}$, and $D_{3}$ are the only three 
secrecy instances of original instance $D$.

Secrecy instances for a given instance $D$ return unmformative answers (1 e. empty or nulls) when asked about the secrecy view contents. Furthermore, we do not want to trivialize too much of the original information in $D$ For this reason we make the secrecy instances minimally depart from $D$ wrt a notion of distance that privileges taking into account the null values In particular, these requirements enforce updates on $D$ that are based only changes of constants by null

\subsection{Privacy Preserving Query Answering}

Our task is to compute secret answers to queries from a given database $D$ that contains secret information The answers to be returned wlll be obtained by querying secrecy instances of $D$ instead of directly querying $D$ There may be several secrecy instances and each of them will return trivial answers when asked about the secrecy views We may consider this collection of secrecy instances as representing some sort of logical database (given through its models), and in consequence, the expected answers are those that are true of all the chosen instances These are usually called certain answers [24]

Definition 32. Let $\mathcal{Q}(\bar{x}) \in L(\Sigma)$ be a conjunctive query A tuple $\bar{a}$ of constants in $\mathcal{U}$ is a secret answer to $\mathcal{Q}$ from $D$ wrt to a set of secrecy views $\mathcal{V}^{s}$ iff $D_{s} k_{N}^{q} \mathcal{Q}[\bar{a}]$ for each $D_{s} \in \operatorname{Sec}\left(D, \mathcal{V}^{s}\right) \quad S A\left(\mathcal{Q}, D, \mathcal{V}^{s}\right)$ denotes the set of all secret answers

Example 4.8. (example 47 contınued) Consıder $\mathcal{Q}(x, z): \exists y(P(x, y) \wedge R(y, z) \wedge$ $y<3)$ For the alternative secrecy instances, we obtain $\mathcal{Q}\left(D_{1}\right)=\{($ null, null $)\}$, $\mathcal{Q}\left(D_{2}\right)=\varnothing$, and $\mathcal{Q}\left(D_{3}\right)=\varnothing$ These answers can be obtained by transforming $\mathcal{Q}$ into a new query $\mathcal{Q}_{N}$ using a methodology proposed in Definition 17 In this case, we obtain 
$\mathcal{Q}_{N}(x, z): \exists y(P(x, y) \wedge R(y, z) \wedge y<3 \wedge y \neq n u l l)$ This query can be evaluated on each of the secrecy instances treating null as any other constant. Finally, we obtain $S A\left(\mathcal{Q}, D,\left\{V_{s}\right\}\right)=\varnothing$.

Notice that, as expected, $S A\left(\mathcal{Q}^{V_{s}}, D\right)=\varnothing$, where $\mathcal{Q}^{V_{s}}$ is the query associated to the secrecy view definition.

Secret answers (SAs) are based on a skeptıcal or cautıous semantics that considers what is true of all the secrecy instances. A more relaxed alternative is a possible or brave semantics, which considers an answer as valid if it is an answer from at least one of the secrecy instances For instance, in Example 4.8, the tuple $P(1,2)$ is a secret answer to the query $P(x, y)$ under the brave semantics, since $P(1,2)$ is an answer to $P(x, y)$ on the secrecy instance $D_{3}$ Simlarly, $R(2,1)$ is a secret answer to $R(x, y)$ under the brave semantics

Notice that the possible answers, that obtained from some secrecy instance, give us more information from the orıginal database than the cautıous answers However, they do not help to solve the privacy problem, because from the secret answers $P(1,2)$ and $R(2,1)$, the user will immediately obtain the contents of the secrecy view.

Even under the skeptical semantics, the user may try to pose queries to obtain sensitive information, as the following example shows

Example 4.9. Consider the new database instance $D=\{P(1,2), P(3,4), R(2,1)$, $R(3,3)\}$, and the secrecy view $V_{s}(x, z) \leftarrow P(x, y), R(y, z)$ This database $D$ has three secrecy instances, indicated in the following table:

\begin{tabular}{|c|c|}
\hline$\imath$ & $D_{\imath}$ \\
\hline 1 & $\{P($ null, 2$), P(3,4), R(2$, null $), R(3,3)\}$ \\
2 & $\{P(1$, null $), P(3,4), R(2,1), R(3,3)\}$ \\
3 & $\{P(1,2), P(3,4), R($ null, 1$), R(3,3)\}$ \\
\hline
\end{tabular}


The user may pose the queries $\mathcal{Q}_{2}(x, y): P(x, y)$ and $\mathcal{Q}_{3}(x, y): R(x, y)$ to try to reconstruct the original database $D$. For the query $\mathcal{Q}_{2}(x, y)$, it holds $\mathcal{Q}_{2}\left(D_{1}\right)=$ $\{($ null, 2$),(3,4)\}, \mathcal{Q}_{2}\left(D_{2}\right)=\{(1$, null $),(3,4)\}$, and $\mathcal{Q}_{2}\left(D_{3}\right)=\{(1,2),(3,4)\}$. In consequence, $S A\left(\mathcal{Q}_{2}, D,\left\{V_{s}\right\}\right)=\{(3,4)\}$

For the query $\mathcal{Q}_{3}(x, y)$, it holds $\mathcal{Q}_{3}\left(D_{1}\right)=\{(2, n u l l),(3,3)\}, \mathcal{Q}_{3}\left(D_{2}\right)=\{(2,1),(3,3)\}$, and $\mathcal{Q}_{3}\left(D_{3}\right)=\{($ null, 1$),(3,3)\}$. In consequence, $S A\left(\mathcal{Q}_{3}, D,\left\{V_{s}\right\}\right)=\{(3,3)\}$.

By combining the secret answers to the subqueries $\mathcal{Q}_{2}$ and $\mathcal{Q}_{3}$ of $\mathcal{Q}^{V_{s}}$, where $\mathcal{Q}^{V_{s}}$ is the query that defines the secrecy view, it is not possible for the user to obtain the extension $\{(1,1)\}$ of $V_{s}[D]$ Actually, if the user poses the queries $\mathcal{Q}_{2}$ and $\mathcal{Q}_{3}$, for him the relations would look like the following

\begin{tabular}{c|c|c|}
\hline$P$ & $X$ & $Y$ \\
\hline & 3 & 4
\end{tabular}

\begin{tabular}{c|c|c|}
\hline$R$ & $Y$ & $Z$ \\
\hline & 3 & 3
\end{tabular}

In this case, any other conjunctive query posed to detect the presence of initial nulls, like $\exists y(P(x, y) \wedge x=n u l l)$, will get an empty set of secret answers, and the user wll not know anything more about the contents of the original instance

Definition 33. Let $\mathcal{V}^{s}$ be a set of secrecy views $V_{s}$ of the form (212). The secrecy answer instance for $\mathcal{V}^{s}$ from $D$ is defined by $D_{\mathcal{V}^{s}}=\left\{R_{\imath}(\bar{t}) \mid R_{\imath} \in \mathcal{R}\right.$ and $\left.\bar{t} \in S A\left(R_{\imath}\left(\bar{x}_{\imath}\right), D, \mathcal{V}^{s}\right)\right\}$

In Definition 33 we are bulding a database instance by collecting the SAs to all the atomic querıes of the form $R(\bar{x})$, with $R \in \mathcal{R}$ This instance has the same schema as the original instance $D$

Example 4.10. (example 49 contmued) Consider the secrecy view $V_{s}(x, z) \leftarrow$ $P(x, y), R(y, z)$ It holds. $D_{\mathcal{V}^{s}}=\{P(3,4)\} \cup\{R(3,3)\}=\{P(3,4), R(3,3)\}$ 
Suppose there is a set of secrecy views defined on the database $D$ that contains sensitive information. We expect that it is not possible for the user to obtain the extensions of the specific secrecy views by combination of secret answers to conjunctive queries The user will try to reconstruct the original instance that builds from the SAs to the queries the instance $D$ and at the end checks if there are answers in the secrecy view evaluated in the constructed instance

The following proposition states that by combination of SAs to queries, there is no way for the user to find out the extensions of the secrecy views evaluated in the original instance $D$

Proposition 2. For every $V_{s}$ of the form $(212)$ in $\mathcal{V}^{s}, S A\left(\mathcal{Q}^{V_{s}}, D, \mathcal{V}^{s}\right)=V_{s}\left[D_{\mathcal{V}^{s}}\right]$

Proof: We know that, $S A\left(\mathcal{Q}^{V_{s}}, D, \mathcal{V}^{s}\right)$ is empty or in all its tuples only null appears

1 First, we consider $S A\left(\mathcal{Q}^{V_{s}}, D, \mathcal{V}^{s}\right)=\varnothing$. This means that there exists at least one secrecy instance $D_{s}$, such that $D_{s} \neq_{N}^{q} \mathcal{Q}^{V_{s}}[\bar{s}[\bar{x} \mid \bar{a}]]^{2}$ By Definition 15, there exists at least one restricted relevant variable $v_{\jmath} \in \mathcal{V}^{R}\left(\mathcal{Q}^{V_{s}}\right)$, and $\bar{s}\left(v_{\jmath}\right)=$ null in $D_{s}$ We can always find one subquery $R_{\imath}\left(\bar{x}_{\imath}\right)$ of $\mathcal{Q}^{V_{s}}$, such that $v_{\jmath} \in \bar{x}_{2}$ Let $\left\{\bar{t}_{1}, ., \bar{t}_{m}\right\}=S A\left(R_{\imath}\left(\bar{x}_{\imath}\right), D, \mathcal{V}^{s}\right)$. The values in $t_{\imath}$ violated $V_{s}$ and associated with $v_{\jmath}$ are $n u l l$ So, by Definition 15 , we can conclude that, $D_{\mathcal{V}^{s}} \bigoplus_{N}^{q} Q^{V_{s}}[\bar{s}[\bar{x} \mid \bar{a}]]$ Thus, $V_{s}\left[D_{\mathcal{V}^{s}}\right]$ is empty.

2. Second, we consider $S A\left(\mathcal{Q}^{V_{s}}, D, \mathcal{V}^{s}\right)$ contain only null tuples This means that for each secrecy instance $D_{s_{\imath}} \in \operatorname{Sec}\left(D, \mathcal{V}^{s}\right)$, it holds $D_{s_{2}} \vDash_{N}^{q}[\bar{s}[\bar{x} \mid \overline{n u l l}]]$ For each subqueries $R_{\imath}\left(\bar{x}_{\imath}\right)$ of $\mathcal{Q}^{V_{s}}$, let $\left\{\bar{t}_{1}, ., \bar{t}_{m}\right\}=S A\left(R_{2}\left(\bar{x}_{\imath}\right), D, \mathcal{V}^{s}\right)$ Since $D_{s_{\imath}} \vDash_{N}^{q}$ $[\bar{s}[\bar{x} \mid \overline{n u l l}]]$, the values in $t_{\imath}$ violated $V_{s}$ and associated with free variables are

\footnotetext{
${ }^{2} \bar{s}$ is a function from the set of variables to the underlying database domain $\mathcal{U}$, such that for the free variables $\left(x_{1}, \quad, x_{n}\right)$ of $\mathcal{Q}$ it holds $\bar{s}\left(x_{\imath}\right)=a_{\imath}$, with $a_{\imath} \in \mathcal{U}$ (cf Definition 13)
} 
null Then, we can conclude that $D_{\mathcal{V}^{s}} \vDash_{N}^{q} Q^{V_{s}}[\bar{s}[\bar{x} \mid \overline{n u l l}]]$ Thus, $V_{s}\left[D_{\mathcal{V}^{s}}\right]$ in all its tuples only null appears.

Notice that we assume that the original database may be incomplete, in the sense that some information is represented using null values A null is used to represent unknown or inapplicable information In consequence, a user who obtains nulls from a query will not know if the nulls were already there or were (virtually) introduced for privacy purposes However, if the user knows the definition of the secrecy views, it is possible for him to combine SAs with the definitıon of the secrecy view to determine the original contents of the view, as shown in the following example

Example 4.11. Consider the secrecy view $V_{s}(x) \leftarrow P(x, y), x=1$, and the database instance $D=\{P(1,1)\}$ The $D$ has the following secrecy instance $D_{s}$ :

\begin{tabular}{c|c|c|}
\hline$P$ & $X$ & $Y$ \\
\hline \multirow{2}{*}{ null } & 1 \\
\cline { 2 - 3 }
\end{tabular}

For the query $\mathcal{Q}(x): \exists y(P(x, y) \wedge x=1)$, the secrecy answer to $\mathcal{Q}(x)$ on $D$ is $\varnothing$ If the user knows there exists one tuple in the original database, by combining the secrecy view definition, he could imply that $(1) \in V_{s}[D]$

In summary, for our solution to work, we are relying on the following assumptions about an external user:

(a) He interacts with a possibly incomplete database.

(b) The interaction is via query answering

(c) The user does not know the secrecy view definitions. 
Based on these assumptions, by Proposition 2, the user can not obtain information about the specific secrecy views, by combination of SAs to any conjunctive queries. Therefore, there is not leakage of sensitive information to the user

\subsection{Summary}

We have introduced a semantıcs of secrecy that considers the possible occurrence of null values in databases Under this semantics, we capture a class of secrecy instances that makes the extensions of the secrecy views either become empty or contain tuples showing only null values Furthermore, the secrecy instances do not depart from the original instance by more than what is needed to protect the secret data So, they minımally differ in a precise sense from the original instance

The queries can be posed against all of secrecy instances simultaneously to obtain secret answers They do not reveal any secret data as defined by the secrecy views The semantics of secret answers (cf Definition 32) is based on the notion of the null query answering introduced in [11] 


\section{Chapter 5}

\section{Secrecy Logic Programs}

As indicated in Chapter 1, we expect the updates leading to the secrecy instances to be virtual Actually, the secrecy instances are more of an auxiliary notion to define the right secrecy semantics In general, we expect not to have to compute all the secrecy instances, materialize them, and then cautiously query them. We would rather stick to the original instance, and use it as it is to obtain the secret answers

One way to approach this problem is via query rewriting Ideally, a query $\mathcal{Q}$ posed to $D$ and expecting secret answers should be rewritten into another query $\mathcal{Q}^{\prime}$ The new query would be posed to $D$ and the usual answers returned by $D$ should be the secret answers to $\mathcal{Q}$ We would like $\mathcal{Q}^{\prime}$ to be still a simple query, that can be easily evaluated. However, the possibility of being able to do this is restricted by the intrinsic complexity of the problem of computing secret answers, which is likely to be higher than polynomial time in data (cf Section 71 ) In consequence, $\mathcal{Q}^{\prime}$ may not be a conjunctive query, actually not even a FO query

An alternative is to specify the secrecy instances in a compact manner, by means of a logical theory, and do reasoning from that theory This will not decrease a high intrinsic complexity, but can be much more efficient than computing all the secrecy instances and querying them in turns. Actually, the secrecy instances for a original database can be specified as the stable models of a disjunctive logic program

The idea is that, given a database instance $D$, and a set $\mathcal{V}^{s}$ of secrecy views of the form (2 12), the secrecy program $\Pi\left(D, \mathcal{V}^{s}\right)$ is constructed in such a way that there is a one-to-one correspondence between the stable models of $\Pi\left(D, \mathcal{V}^{s}\right)$ and the secrecy 
instances of $D$.

The secrecy program uses annotation constants with the intended, informal semantics shown in the table below. The annotations are used to keep track of virtual updates, 1 e. of old and new data.

\begin{tabular}{|c|c|c|}
\hline Annotation & Atom & The tuple $R(\bar{a})$ \\
\hline $\mathbf{b}_{\mathbf{u}}$ & $R\left(\bar{a}, \mathbf{b}_{\mathbf{u}}\right)$ & has already been updated (old data) \\
$\mathbf{a}_{\mathbf{u}}$ & $R\left(\bar{a}, \mathbf{a}_{\mathbf{u}}\right)$ & 1s updated tuple in database (new data) \\
$\mathbf{t}$ & $R(\bar{a}, \mathbf{t})$ & is new or old tuple \\
$\mathbf{s}$ & $R(\bar{a}, \mathbf{s})$ & stays in the final database \\
\hline
\end{tabular}

Table 5.1: Annotation Constants

In a program we will find each database predicate $R \in \mathcal{R}$ together with its new version $R$ of it, that contains an extra argument that is used to place an annotation constant The 1 dea is to use logic rules to specify and capture how a database violates secrecy, and how the database can become secret with respect to a set $\mathcal{V}^{s}$ of secrecy views via null-based updates

Actually, each atom of the form $R(\bar{a})$ will receive one of the constants in Table 51 In $R\left(\bar{a}, \mathbf{b}_{\mathbf{u}}\right)$, annotation $\mathbf{b}_{\mathbf{u}}$ means that the atom has already been updated, and $\mathbf{a}_{\mathbf{u}}$ should appear in the new, updated atom. For example, consider a tuple $R(a, b) \in D$ A new tuple $R(a, n u l l)$ is obtained via the update of $b$ to $n u l l$. Therefore, $R\left(a, b, \mathbf{b}_{\mathbf{u}}\right)$ denotes the old atom before updating, whlle $R\left(a, n u l l, \mathbf{a}_{\mathbf{u}}\right)$ denotes the new atom after the update

Annotations are performed according to the following sequential steps· First, each ground atom $R(\bar{a})$ from the database becomes a fact in $\Pi\left(D, \mathcal{V}^{s}\right)$ Next, for each secrecy view definition, a disjunctive rule is constructed in such a way that the body of the rule captures the violation condition for the secrecy, and the head describes how to restore secrecy by updating the corresponding tuples The update is achieved by using annotation $\mathbf{a}_{\mathbf{u}}$ 
Example 5.1. As an illustration, consider a schema with relations $P(X, Y), R(Y, Z)$, and the following secrecy view

$$
V_{s}(x, z) \leftarrow P(x, y), R(y, z), y<3
$$

The disjunctive program rule:

$$
\begin{array}{r}
\left(P_{-}\left(n u l l, y, \mathbf{a}_{\mathbf{u}}\right) \wedge R\left(y, \text { null }, \mathbf{a}_{\mathbf{u}}\right)\right) \vee P_{-}\left(x, n u l l, \mathbf{a}_{\mathbf{u}}\right) \vee R\left(n u l l, z, \mathbf{a}_{\mathbf{u}}\right) \\
\leftarrow P(x, y), R(y, z), y<3, y \neq n u l l, \operatorname{aux}(x, z),
\end{array}
$$

states that if the given database is not already a secrecy instance, then secrecy is restored by either updating values in the combination attribute $Y$ with nulls, or values in the secrecy attributes $X$ and $Z$ with nulls, simultaneously

In general, disjunctive logic programs do not allow for conjunctions in the head Therefore, the intended head rule of $(52)$ generates two rules

$$
\begin{aligned}
& P_{-}\left(n u l l, y, \mathbf{a}_{\mathbf{u}}\right) \vee P_{-}\left(x, n u l l, \mathbf{a}_{\mathbf{u}}\right) \vee R\left(n u l l, z, \mathbf{a}_{\mathbf{u}}\right) \leftarrow B o d y .^{1} \\
& R_{-}\left(y, n u l l, \mathbf{a}_{\mathbf{u}}\right) \vee P_{-}\left(x, n u l l, \mathbf{a}_{\mathbf{u}}\right) \vee R\left(n u l l, z, \mathbf{a}_{\mathbf{u}}\right) \leftarrow B o d y
\end{aligned}
$$

Furthermore, we need to restore secrecy only if the given database is not already a secrecy instance, which happens when all the combinatıon attributes are not null, the secrecy attributes are not simultaneously null, and formula $\varphi$ is true For the secrecy view (5.1), the secrecy attributes are $X$ and $Z$, and the combinability attribute is $Y$. We use the following auxiliary rules to capture the idea of the secrecy attributes are not simultaneously null.

$$
\begin{aligned}
& \operatorname{aux}(x, z) \leftarrow P(x, y), R(y, z), y<3, x \neq n \text { null. } \\
& \operatorname{aux}(x, z) \leftarrow P(x, y), R(y, z), y<3, z \neq n u l l .
\end{aligned}
$$

\footnotetext{
${ }^{1}$ The body of rule, denoted by Body, remains the same as the one of ( 52 )
} 
The annotation constant $\mathbf{t}$ is introduced in order to solve the interaction problem among secrecy views Thus, it becomes highly significant in cases when the updated instance may still not be secrecy according another secrecy view. For example, $P\left(x, n u l l, \mathbf{a}_{\mathbf{u}}\right)$ is generated by using Rule (53), which is not secrecy with respect to the different secrecy view $V_{s}(x) \leftarrow P(x, y)$ So, we need to keep updating the instance wrt $V_{s}(x) \leftarrow P(x, y)$ The aftermath is that the bodies of rules (53) and (54) have to be modified to:

$$
P_{-}(x, y, \mathbf{t}), R(y, z, \mathbf{t}), y<3, y \neq n u l l, a u x(x, z),
$$

and the program rules (5 5) and (56) have to be changed to

$$
\begin{aligned}
& \operatorname{aux}(x, z) \leftarrow P_{-}(x, y, \mathbf{t}), R_{-}(y, z, \mathbf{t}), y<3, x \neq n u l l, \\
& \operatorname{aux}(x, z) \leftarrow P_{-}(x, y, \mathbf{t}), R_{-}(y, z, \mathbf{t}), y<3, z \neq n u l l,
\end{aligned}
$$

where the atom $P_{-}(x, y, \mathbf{t})$ becomes true if ether $P(x y)$ or $P_{-}\left(x, y, \mathbf{a}_{\mathbf{u}}\right)$ are true Similarly for the atom $R(y, z, \mathbf{t})$.

Moreover, we need to collect the tuples in the database that have already been updated and (virtually) no longer exıst in the database. This is acheved by using the following rules:

$$
\begin{gathered}
P_{-}\left(x, y \cdot \mathbf{b}_{\mathbf{u}}\right) \leftarrow P_{-}(x, y, \mathbf{t}), R_{-}(y, z, \mathbf{t}), y<3, y \neq n u l l, a u x(x, z), P_{-}\left(n u l l, y, \mathbf{a}_{\mathbf{u}}\right), x \neq n u l l \\
R\left(y, z . \mathbf{b}_{\mathbf{u}}\right) \leftarrow P_{-}(x, y, \mathbf{t}), R(y, z, \mathbf{t}), y<3, y \neq n u l l, a u x(x, z), R\left(y . n u l l, \mathbf{a}_{\mathbf{u}}\right), z \neq n u l l . \\
P_{-}\left(x, y, \mathbf{b}_{\mathbf{u}}\right) \leftarrow P_{-}(x, y, \mathbf{t}), R(y, z, \mathbf{t}), y<3, y \neq n u l l, a u x(x, z), P_{-}\left(x, n u l l, \mathbf{a}_{\mathbf{u}}\right) \\
R\left(y, z, \mathbf{b}_{\mathbf{u}}\right) \leftarrow P_{-}(x, y, \mathbf{t}), R(y, z, \mathbf{t}), y<3, y \neq n u l l, a u x(x, z), R\left(n u l l, z, \mathbf{a}_{\mathbf{u}}\right) .
\end{gathered}
$$

Finally, atoms with annotation constant $\mathbf{s}$ are the ones that become true in the secrecy instances. This constant is used to read off the database atoms in the secrecy 
instances So, the secrecy instances are obtamed by restrictions of the models of the program to those atoms with the $\mathbf{s}$ annotation by using following rules.

$$
\begin{gathered}
P_{-}(x, y, \mathbf{s}) \leftarrow P_{-}(x, y, \mathbf{t}), \operatorname{not} P_{-}\left(x, y, \mathbf{b}_{\mathbf{u}}\right) . \\
R_{(}(x, y, \mathbf{s}) \leftarrow R_{-}(x, y, \mathbf{t}), \operatorname{not} R\left(x, y, \mathbf{b}_{\mathbf{u}}\right) .
\end{gathered}
$$

Before presenting the formal and general definition of logic program, we need some concepts For an atom of the form $R(\bar{x})$, change $(R(\bar{x}), \bar{y}, t)$, with $\bar{y}=\left\{y_{1}, \quad, y_{n}\right\}$ and $\bar{y} \subseteq \bar{x}$, represents changing each variable $y_{\imath} \in \bar{x}$ to $t$, with $t$ is a variable or domain constant Let us recall that a secrecy view $V_{s}$ is of the form

$$
V_{s}(\bar{x}) \leftarrow R_{1}\left(\bar{x}_{1}\right), \quad, R_{n}\left(\bar{x}_{n}\right), \varphi,
$$

For each $R_{\imath}\left(\bar{x}_{\imath}\right)$ in the body of the secrecy view, if $\left(\bar{x}_{\imath} \cap \mathcal{C}\left(V_{s}\right)\right) \neq \varnothing$, let $\mathcal{C P}\left(V_{s}\right)=$ $\left\{\right.$ change $\left(R_{\imath}\left(\bar{x}_{\imath}\right), y, n u l l\right)$, for each $\left.y \in\left(\bar{x}_{\imath} \cap \mathcal{C}\left(V_{s}\right)\right)\right\}$, and if $\left(\bar{x}_{\imath} \cap \mathcal{S}\left(V_{s}\right)\right) \neq \varnothing$, let $\mathcal{S P}\left(V_{s}\right)=\left\{\right.$ change $\left(R_{\imath}\left(\bar{x}_{\imath}\right), \bar{y}\right.$, null $)$, for $\left.\bar{y}=\left(\bar{x}_{\imath} \cap \mathcal{C}\left(V_{s}\right)\right)\right\} \quad$ The idea behind these two notions is to characterize the LHS of the disjunctive program rule, which is used to restore secrecy

Example 5.2. Consider the following secrecy view $V_{s}(x, z, w) \leftarrow P(x, y), Q(y, z, w)$ By Definition 24, $C\left(V_{s}\right)=\{P[2], Q[1]\}$, and $S\left(V_{s}\right)=\{P[1], Q[2], Q[3]\}$ according to Defintion $25 \mathcal{C P}\left(V_{s}\right)=\{P(x, n u l l), Q(n u l l, z, w)\}$, and $\mathcal{S P}\left(V_{s}\right)=\{P(n u l l, y)$, $Q(y, n u l l, n u l l)\}$

Definition 34. Given a database instance $D$, a set $\mathcal{V}^{s}$ of secrecy views $V_{s}$ s of the form (2 12), the secrecy program $\Pi\left(D, \mathcal{V}^{s}\right)$ contains the following rules: 
1 Facts: $R(\bar{a})$ for each atom $R(\bar{a}) \in D$.

2. For every $V_{s}$ of the form (212), let $\mathcal{S P}\left(V_{s}\right)=\left\{R^{1}\left(\bar{x}_{1}\right), ., R^{a}\left(\bar{x}_{a}\right)\right\}$, and $\mathcal{C} P\left(V_{s}\right)=$ $\left\{R^{1}\left(\bar{x}_{1}\right), \quad, R^{b}\left(\bar{x}_{b}\right)\right\}$ The rules:

(a) If $\mathcal{S}\left(V_{s}\right) \cap \mathcal{C}\left(V_{s}\right) \neq \varnothing$, the rule:

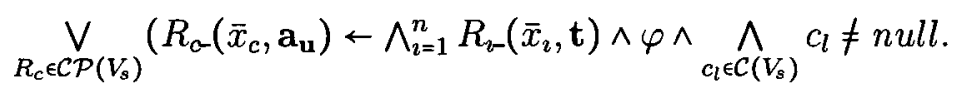

(b) If $\mathcal{S}\left(V_{s}\right) \cap \mathcal{C}\left(V_{s}\right)=\varnothing$, the rule

$$
\begin{aligned}
R_{s \jmath}\left(\bar{x}_{s \jmath}, \mathbf{a}_{\mathbf{u}}\right) \vee & {\underset{R}{R_{c} \in \mathcal{C P}\left(V_{s}\right)}}^{\vee}\left(R_{c-}\left(\bar{x}_{c}, \mathbf{a}_{\mathbf{u}}\right)\right. \\
& \leftarrow \bigwedge_{\imath=1}^{n} R_{\imath-(}\left(\bar{x}_{\imath}, \mathbf{t}\right) \wedge \varphi \wedge \bigwedge_{c_{l} \in \mathcal{C}\left(V_{s}\right)} c_{l} \neq \text { null } \wedge \text { aux } V_{V_{s}}(\bar{s}),
\end{aligned}
$$

with $R_{s \jmath} \in \mathcal{S P}\left(V_{s}\right)$, and $1 \leq \jmath \leq a$

Plus the auxiliary rules

If $\mathcal{S}\left(V_{s}\right)$ is $s^{1}, \quad, s^{k}$, then for $1 \leqslant \imath \leqslant k$,

$\operatorname{aux}_{V_{s}}(\overline{\mathbf{s}}) \leftarrow \wedge_{\imath=1}^{n} R_{\imath}\left(\bar{x}_{\imath}, \mathbf{t}\right) \wedge \varphi \wedge s_{\imath} \neq n u l l$, where $\bar{s}=\bigcup s_{\imath}$

3. The collection rules

$$
\begin{aligned}
& R_{s \jmath-}\left(\bar{x}_{s \jmath}, \mathbf{b}_{\mathbf{u}}\right) \leftarrow \bigwedge_{\imath=1}^{n} R_{\imath-}\left(\bar{x}_{\imath}, \mathbf{t}\right) \wedge \varphi \wedge a u x_{V_{s}}(\bar{s}) \wedge \bigwedge_{c_{l} \in \mathcal{C}\left(V_{s}\right)} c_{l} \neq n u l l \wedge R_{s \jmath-}\left(\bar{x}_{s \jmath}, \mathbf{a}_{\mathbf{u}}\right) \wedge \\
& \bigwedge_{s_{l} \in\left(S\left(V_{s}\right) n_{x_{s \jmath}}\right)} s_{l} \neq n u l l, \text { for } R_{s \jmath} \in \mathcal{S P}\left(V_{s}\right), \text { and } 1 \leq \jmath \leq a \\
& R_{c k-}\left(\bar{x}_{c k}, \mathbf{b}_{\mathbf{u}}\right) \leftarrow \bigwedge_{\imath=1}^{n} R_{\imath-}\left(\bar{x}_{\imath}, \mathbf{t}\right) \wedge \varphi \wedge a u x_{V_{s}}(\bar{s}) \wedge \bigwedge_{c_{l} \in \mathcal{C}\left(V_{s}\right)} c_{l} \neq n u l l \wedge \\
& R_{c k-}\left(\bar{x}_{c k}, \mathbf{a}_{\mathbf{u}}\right), \text { for } R_{c k} \in \mathcal{C P}\left(V_{s}\right), \text { and } 1 \leq k \leq b .
\end{aligned}
$$

4 For each predicate $R \in \mathcal{R}$, the annotation rules

$$
R(\bar{x}, \mathbf{t}) \leftarrow R(\bar{x}) . \quad R(\bar{x}, \mathbf{t}) \leftarrow R\left(\bar{x}, \mathbf{a}_{\mathbf{u}}\right)
$$

5 For each predicate $R \in \mathcal{R}$, the interpretation rule

$$
R_{-}(\bar{x}, \mathbf{s}) \leftarrow R_{-}(\bar{x}, \mathbf{t}), \operatorname{not} R\left(\bar{x}, \mathbf{b}_{\mathbf{u}}\right)
$$

The rules in 1. establish the program facts which are the elements of the database The rules in 2. are the most important and express how to restore secrecy. The body 
of the rule (right-hand side) will be true if the database instance is not a secrecy instance, and with the left-hand side captures the intended way of restoring secrecy. The rules in 3 . collect the tuples in the database that have already been updated and (virtually) no longer exist in the database. Rules 4 capture the atoms that are part of the database or updated atoms in the process of restoring secrecy. Rules in 5 collect the tuples that stay in the final state of the updated database.

Example 5.3. (example 47 continued) Consider $D=\{P(1,2), R(2,1)\}$ and the secrecy view $V_{s}(x, z) \leftarrow P(x, y), R(y, z), y<3$ The secrecy instance program $\Pi\left(D,\left\{V_{s}\right\}\right)$ is as follows.

$1 \quad P(1,2), R(2,1)$. (the initial database contents)

$2 \quad P_{-}\left(n u l l, y, \mathbf{a}_{\mathbf{u}}\right) \vee P_{-}\left(x, n u l l, \mathbf{a}_{\mathbf{u}}\right) \vee R\left(n u l l, z, \mathbf{a}_{\mathbf{u}}\right)$

$$
\leftarrow P_{-}(x, y, \mathbf{t}), R(y, z, \mathbf{t}), y<3, y \neq n u l l, a u x(x, z)
$$

$R\left(y, n u l l, \mathbf{a}_{\mathbf{u}}\right) \vee P_{-}\left(x, n u l l, \mathbf{a}_{\mathbf{u}}\right) \vee R\left(n u l l, z, \mathbf{a}_{\mathbf{u}}\right)$

$$
\leftarrow P_{-}(x, y, \mathbf{t}), R(y, z, \mathbf{t}), y<3, y \neq n u l l, \operatorname{aux}(x, z) .
$$

$\operatorname{aux}(x, z) \leftarrow P_{-}(x, y, \mathbf{t}), R_{-}(y, z, \mathbf{t}), y<3, x \neq$ null

aux $(x, z) \leftarrow P_{-}(x, y, \mathbf{t}), R_{-}(y, z, \mathbf{t}), y<3, z \neq n$ null

$3 \quad P_{-}\left(x, y, \mathbf{b}_{\mathbf{u}}\right) \leftarrow P_{-}(x, y, \mathbf{t}), R_{-}(y, z, \mathbf{t})$,

$$
y<3, y \neq \operatorname{null}, \text { aux }(x, z), P_{-}\left(\text {null }, y, \mathbf{a}_{\mathbf{u}}\right), x \neq n u l l .
$$

$R\left(y, z, \mathbf{b}_{\mathbf{u}}\right) \leftarrow P_{-}(x, y, \mathbf{t}), R(y, z, \mathbf{t})$,

$$
y<3, y \neq n u l l, \operatorname{aux}(x, z), R_{-}\left(y, n u l l, \mathbf{a}_{\mathbf{u}}\right), z \neq n u l l
$$

$P_{-}\left(x, y, \mathbf{b}_{\mathbf{u}}\right) \leftarrow P_{-}(x, y, \mathbf{t}), R(y, z, \mathbf{t}), y<3, y \neq n u l l, a u x(x, z), P_{-}\left(x, n u l l, \mathbf{a}_{\mathbf{u}}\right)$.

$R\left(y, z, \mathbf{b}_{\mathbf{u}}\right) \leftarrow P_{-}(x, y, \mathbf{t}), R_{-}(y, z, \mathbf{t}), y<3, y \neq n u l l$, aux $(x, z), R\left(n u l l, z, \mathbf{a}_{\mathbf{u}}\right)$.

$4 \quad P_{-}(x, y, \mathbf{t}) \leftarrow P(x, y) \quad P_{-}(x, y, \mathbf{t}) \leftarrow P_{-}\left(x, y, \mathbf{a}_{\mathbf{u}}\right)$

$R(x, y, \mathbf{t}) \leftarrow R(x, y) \quad R(x, y, \mathbf{t}) \leftarrow R\left(x, y, \mathbf{a}_{\mathbf{u}}\right)$.

$5 \quad P_{-}(x, y, \mathbf{s}) \leftarrow P_{-}(x, y, \mathbf{t})$, not $P_{-}\left(x, y, \mathbf{b}_{\mathbf{u}}\right)$.

$R_{\sim}(x, y, \mathrm{~s}) \leftarrow R(x, y, \mathbf{t})$, not $R\left(x, y, \mathbf{b}_{\mathbf{u}}\right)$ 
The secrecy instances are in one-to-one correspondence with the stable models of program $\Pi\left(D, \mathcal{V}^{s}\right)$. Given a model, the associated secrecy instance is obtained by collecting the atoms that are annotated with s.

The program can be evaluated, for example, using the $D L V$ system that computes the disjunctive stable models semantics It offers a nice and effective interface to commercial DBMSs [30]

Example 5.4. (example 5.3 continued) The program has three stable models (the facts of the program are omitted for simplicity)

$$
\begin{aligned}
& M_{1}=\left\{P(1,2, \mathbf{t}), R(2,1, \mathbf{t}), \operatorname{aux}(1,1), \underline{P(1,2, \mathbf{s})}, R\left(2,1, \mathbf{b}_{\mathbf{u}}\right), R\left(n u l l, 1, \mathbf{a}_{\mathbf{u}}\right)\right. \text {, } \\
& R(\text { null }, 1, \mathbf{t}), \underline{R(n u l l, 1, \mathbf{s})}\} \\
& M_{2}=\left\{P(1,2, \mathbf{t}), R(2,1, \mathbf{t}), \operatorname{aux}(1,1), P\left(1.2, \mathbf{b}_{\mathbf{u}}\right) . \underline{R(2,1, \mathbf{s})}, P\left(1 . n u l l, \mathbf{a}_{\mathbf{u}}\right)\right. \text {, } \\
& P(1, \text { null }, \mathbf{t}), P(1, \text { null }, \mathbf{s})\} \text {. } \\
& M_{3}=\left\{P(1,2, \mathbf{t}), R(2,1, \mathbf{t}), \operatorname{aux}(1,1), P\left(1,2, \mathbf{b}_{\mathbf{u}}\right), R\left(2,1, \mathbf{b}_{\mathbf{u}}\right), P\left(n u l l, 2, \mathbf{a}_{\mathbf{u}}\right)\right. \text {, } \\
& R\left(2 . n u l l, \mathbf{a}_{\mathbf{u}}\right), P(n u l l, 2, \mathbf{t}), R(2, n u l l, \mathbf{t}), \text { aux }(1, n u l l), \text { aux }(n u l l, 1), \\
& \underline{P(n u l l, 2, \mathbf{s})}, \underline{R(2, n u l l, \mathbf{s})}\}
\end{aligned}
$$

The secrecy instances select the underlmed atoms. $D_{1}=\{P(1,2), R($ null, 1$)\}, D_{2}=$ $\{P(1, n u l l), R(2,1)\}$, and $D_{3}=\{P(n u l l, 2), R(2, n u l l)\}$. As expected, these are the secrecy instances obtained in Example 47.

If we want to obtain the secret answers to a FO conjunctive query $\mathcal{Q}$ written in the language of $L(\Sigma)$, it is not necessary to explicitly compute all the stable models Instead, the query can be posed directly to the models of the reparr program, 1.e. to the secrecy instances, and answered according to the skeptical semantics. This wll return the secret answers to the query. Of course, the query has to be transformed as a top-layer program This transformation is done in the following way: 
1. Conjunctive query $\mathcal{Q}$ of the form (2.1) first has to be rewritten as the query $\mathcal{Q}_{N}$ using the methodology introduced in Definition 17.

2. The new query $\mathcal{Q}_{N}$ is transformed into a query written as a logic program $\Pi(\mathcal{Q})$, which is a stratıfied Datalog program $[33,4]$. $\Pi(\mathcal{Q})$ contains a predicate Ans, to collect the final query answers.

3. Each database predicate $R(\bar{x})$ in $\Pi(\mathcal{Q})$ is replaced by $R(\bar{x}, \mathbf{s})$

4. The query program $\Pi(\mathcal{Q})$ is combined with the secrecy program $\Pi\left(D, \mathcal{V}^{s}\right)$ into a new program $\Pi$

5. The extension of the answer predicate Ans in the intersection of all stable models of $\Pi$ contains exactly the secret answers If $\mathcal{Q}$ is a boolean query, yes is a secret answer if Ans is in the intersection of all the models Otherwise, the secret answer is no

Example 5.5. (example 54 contınued) We want the secret answers to the conjunctive query $\mathcal{Q}(x, z): \exists y(P(x, y) \wedge R(y, z) \wedge y<3)$ This requires first rewriting it as the query $\mathcal{Q}_{N}$ obtained in Example 47 . This new query can be evaluated against instances with null treated as any other constant. Now, query $\mathcal{Q}_{N}$ can be transformed into a query program $\Pi(\mathcal{Q}): A n s(x, z) \leftarrow P(x, y, \mathbf{s}), R(y, z, \mathbf{s}), y<3, y \neq n u l l$, which has to be evaluated in combination with the program in Example 53 , under the skeptical semantics In this evaluatıon, null is treated as an ordinary constant. The stable models of program $\Pi$ are the stable models of $\Pi\left(D, \mathcal{V}^{s}\right)$ expanded by the answers to the query:

$$
\begin{aligned}
& M_{1}=M_{1} \cup \varnothing, \\
& M_{2}=M_{2} \cup \varnothing, \\
& M_{3}=M_{3} \cup\{\text { Ans }(\text { null }, \text { null })\}
\end{aligned}
$$


Since secret answers are those we get from all the possible secrecy instances, the answer to this query $\mathcal{Q}$ is $\varnothing$

On the other hand, for the boolean query $\mathcal{Q}_{2} . \exists x y z(P(x, y) \wedge R(y, z))$, the query program $\Pi\left(\mathcal{Q}_{2}\right)$ is: $A n s \leftarrow P(x, y, \mathbf{s}), R(y, z, \mathbf{s}), y \neq$ null The stable models of program $\Pi$ are the stable models of $\Pi\left(D, \mathcal{V}^{s}\right)$ expanded by the answers to the query:

$$
\begin{aligned}
& M_{1}=M_{1} \cup\{\}, \\
& M_{2}=M_{2} \cup\{\}, \\
& M_{3}=M_{3} \cup\{\text { Ans }\}
\end{aligned}
$$

The secret answer to this boolean query $\mathcal{Q}_{2}$ is no.

We have successfully experimented with the DLV system for computing secret answers with respect to above examples.

\subsection{Optimizing Query Evaluation from Secrecy Programs}

As indicated above, we compute secret answers by evaluating the combination of the secrecy and query programs These programs are constructed considering all the database predicates and facts, which contain more information than necessary to obtain secret answers In fact, in most of cases, only a subset of database predicates and facts are needed to compute answers to a specific query. So bringing the whole database into DLV is inefficient. Therefore, we need to optimize the secrecy programs by generating only those parts that are relevant for answering the query, and importing only the relevant data facts for computing answers

Our optimization approach starts by capturing the relevant database predicates for a specific query. This is done by analyzing the relatıonship between predicates in the secrecy view definitions and queries. Intuitively, rules in the secrecy programs that do not involve any relevant predicate are eliminated So the relevant predicates are used for pruning secrecy programs. As a result, programs are smaller than the 
original ones. Next, our optimization is to apply the bult-ins in the query to the relevant database predicates to retrieve the appropriate data In this manner, we import only a subset of data rather than retrieving all the data. Our optımization to the evaluation of programs make query processing more efficient In Chapter 6 we perform experiments to show the effectiveness

\subsubsection{Relevant Predicates}

In the area of consistent query answering (CQA), an optimization technique is presented that captures the relevant predicates [13] to compute consistent answers from the repair programs

Given a query, there might be a set of integrity constraints that are not relevant to the query, i e their satisfaction or not does not affect the answers to the query In order to capture the relevant ICs, the relevant predicates are defined and detected by analyzing the relationship between predicates in queries and ICs This is done by means of a dependency graph Basically, a dependency graph for ICs represents the dependency relationship among predicates in ICs, i e transitive dependencies between predicates From the graph, it can easly find the relevant predicates that are needed to answer a query

In this research we adopted the idea of the dependency graph approach introduced In $[13,14,15]$ to capture the relevant predicates for our secrecy programs. The following is definition of the dependency graph for a set of secrecy views

Definition 35. The dependency graph, denoted by $\mathcal{G}\left(\mathcal{V}^{s}\right)$ for a set $\mathcal{V}^{s}$ of secrecy views $V_{s}$ s of the form (2.12) is defined as follows for each database predicate $R \in \mathcal{R}$ appearing in $\mathcal{V}^{s}$ is a vertex, and there is an edge $\left(R_{\imath}, R_{\jmath}\right)$ between $R_{\imath}$ and $R_{\jmath}$ iff there exists a secrecy view $V_{s} \in \mathcal{V}^{s}$ such that both $R_{\imath}$ and $R_{\jmath}$ appeals in the body of $V_{s}$

Example 5.6. Figure 5.1 illustrates the dependency graph $\mathcal{G}\left(\mathcal{V}^{s}\right)$ for the set $\mathcal{V}^{s}$ of 
secrecy views' $\left\{V_{s}(x) \leftarrow P(x, y), Q(x, y), V_{s}(x) \leftarrow S(x, y), V_{s}(y) \leftarrow S(x, y), R(y)\right\}$

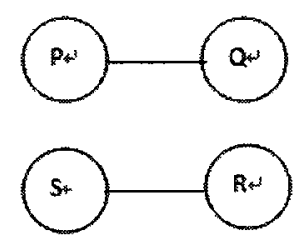

Figure 51 Dependency Graph

Definition 36. The set of relevant predıcates for secret answers to a query $\mathcal{Q}$ with respect to a set $\mathcal{V}^{s}$ of secrecy views $V_{s}$ s of the form (212), denoted by $\operatorname{Rel}\left(\mathcal{Q}, \mathcal{V}^{s}\right)$, contains the following:

(a) For each predicate $R \in \mathcal{Q}, R$ is in $\operatorname{Rel}\left(\mathcal{Q}, \mathcal{V}^{s}\right)$,

(b) For a predicate $R^{\prime}$ such that there is a path between $\left(R, R^{\prime}\right)$ in the dependency $\operatorname{graph} \mathcal{G}\left(\mathcal{V}^{s}\right), R^{\prime} \in \operatorname{Rel}\left(\mathcal{Q}, \mathcal{V}^{s}\right)$.

Proposition 3. Let $\Pi\left(D, \mathcal{V}^{s}, \mathcal{Q}\right)$ be the combination of the secrecy and query programs, and $\Pi\left(D, \mathcal{V}^{s}, \mathcal{Q}\right) \downarrow \mathcal{Q}$ denotes the same as program $\Pi\left(D, \mathcal{V}^{s}, \mathcal{Q}\right)$ except that the former contains only rules for each predicate $R \in \operatorname{Rel}\left(\mathcal{Q}, \mathcal{V}^{s}\right)$. It holds that $\Pi\left(D, \mathcal{V}^{s}, \mathcal{Q}\right) \downarrow \mathcal{Q}$ and $\Pi\left(D, \mathcal{V}^{s}, \mathcal{Q}\right)$ retreve the same secret answers to query $\mathcal{Q}$

We now sketch the proof, which is based on the same basic idea as the proof in [13, Proposition 5 6]

Proof of Proposition 3: Obviously, the program $\Pi\left(D, \mathcal{V}^{s}, \mathcal{Q}\right)$ can be split into two $\log 1 \mathrm{c}$ programs $\Pi_{R}\left(D, \mathcal{V}^{s}, \mathcal{Q}\right)$, and $\Pi_{N R}\left(D, \mathcal{V}^{s}, \mathcal{Q}\right)$, respectively. Let $\Pi_{R}\left(D, \mathcal{V}^{s}, \mathcal{Q}\right)=$ $\Pi\left(D, \mathcal{V}^{s}, \mathcal{Q}\right) \downarrow \mathcal{Q}$ The models of program $\Pi\left(D, \mathcal{V}^{s}, \mathcal{Q}\right)$, denoted by $\mathcal{S} \mathcal{M}\left(\Pi\left(D, \mathcal{V}^{s}, \mathcal{Q}\right)\right)$, 
can be obtained by $\cup_{M}\left(M \cup \mathcal{S} \mathcal{M}\left(\Pi_{N R}\left(D, \mathcal{V}^{s}, \mathcal{Q}\right)\right)\right.$, for each stable model $M$ of $\Pi_{R}(D$, $\left.\mathcal{V}^{s}, \mathcal{Q}\right)$. Since the program $\Pi_{N R}\left(D, \mathcal{V}^{s}, \mathcal{Q}\right)$ does not have rules whose predicates are related with the query predicates, we can not find Ans predicate in any of stable models of $\Pi_{N R}\left(D, \mathcal{V}^{s}, \mathcal{Q}\right)$. The Ans predicate can be obtained only by the program $\Pi_{R}\left(D, \mathcal{V}^{s}, \mathcal{Q}\right)$ Therefore, all the stable models of $\Pi\left(D, \mathcal{V}^{s}, \mathcal{Q}\right)$ contain the same Ans predicate as $\Pi_{R}\left(D, \mathcal{V}^{s}, \mathcal{Q}\right)$. Thus, $\Pi\left(D, \mathcal{V}^{s}, \mathcal{Q}\right) \downarrow \mathcal{Q}$ and $\Pi\left(D, \mathcal{V}^{s}, \mathcal{Q}\right)$ retrieve the same secret answers to query $\mathcal{Q}$

Example 5.7. (example 5.6 continued) Consider the query $\mathcal{Q}: A n s(x) \leftarrow S(x, y, \mathbf{s})$, and the database $D=\{S(1,2), R(2), P(1,1), Q(1,1)\}$. The set of relevant predicates for the query $\mathcal{Q}$ is $\operatorname{Rel}\left(\mathcal{Q}, \mathcal{V}^{s}\right)=\{S, R\}$. Therefore, program $\Pi\left(D, \mathcal{V}^{s}, \mathcal{Q}\right) \downarrow \mathcal{Q}$ contains the following rules.

$$
\begin{aligned}
& S(1,2) \quad R(2) \\
& S_{-}(x, y, \mathbf{t}) \leftarrow S_{-}\left(x, y, \mathbf{a}_{\mathbf{u}}\right) \quad S_{-}(x, y, \mathbf{t}) \leftarrow S(x, y) \\
& R(x, \mathbf{t}) \leftarrow R_{-}\left(x, \mathbf{a}_{\mathbf{u}}\right) \quad R(x, \mathbf{t}) \leftarrow R(x) . \\
& S_{-}\left(x, n u l l, \mathbf{a}_{\mathbf{u}}\right) \vee R\left(n u l l, \mathbf{a}_{\mathbf{u}}\right) \leftarrow S_{-}(x, y, \mathbf{t}), R_{-}(y, \mathbf{t}), y \neq n u l l \\
& S_{-}\left(x, y, \mathbf{b}_{\mathbf{u}}\right) \leftarrow S_{-}(x, y, \mathbf{t}), R_{-}(y, \mathbf{t}), S_{-}\left(x, n u l l, \mathbf{a}_{\mathbf{u}}\right), y \neq n u l l \\
& R\left(y, \mathbf{b}_{\mathbf{u}}\right) \leftarrow S_{-}(x, y, \mathbf{t}), R(y, \mathbf{t}), R_{-}\left(n u l l, \mathbf{a}_{\mathbf{u}}\right), y \neq n u l l . \\
& S_{-}\left(n u l l, y, \mathbf{a}_{\mathbf{u}}\right) \leftarrow S_{-}(x, y, \mathbf{t}), \text { aux } S(x) \\
& \text { auxS }(x) \leftarrow S_{-}(x, y, \mathbf{t}), x \neq n u l l \\
& S_{-}\left(x, y, \mathbf{b}_{\mathbf{u}}\right) \leftarrow S_{-}(x, y, \mathbf{t}), S_{-}\left(n u l l, y, \mathbf{a}_{\mathbf{u}}\right), \text { auxS }(x) \\
& S_{-}(x, y, \mathbf{s}) \leftarrow S_{-}(x, y, \mathbf{t}), \text { not } S_{-}\left(x, y, \mathbf{b}_{\mathbf{u}}\right) . \\
& R_{-}(x, \mathbf{s}) \leftarrow R_{-}(x, \mathbf{t}), \text { not } R\left(x, \mathbf{b}_{\mathbf{u}}\right) \\
& \text { Ans }(x) \leftarrow S_{(}(x, y, \mathbf{s}) .
\end{aligned}
$$

The program $\Pi\left(D, \mathcal{V}^{s}, \mathcal{Q}\right) \downarrow \mathcal{Q}$ has two stable models•

$$
\begin{aligned}
& \mathcal{M}_{1}=\{S(\text { null }, \text { null }, \mathbf{s}), R(2, \mathbf{s}), \text { Ans }(\text { null })\} \\
& \mathcal{M}_{2}=\{S(\text { null }, 2, \mathbf{s}), R(1, \mathbf{s}), \text { Ans }(\text { null })\}
\end{aligned}
$$


Since there is Ans-atoms in common, the secret answer to this query $\mathcal{Q}$ is $(n u l l)$, as expected Notice that, rules and database facts that do not involve relevant predicates are not generated. Thus, secret answers are obtained by running the smaller secrecy program together with the query program in DLV system

\subsubsection{Relevant Facts}

In the former approach, all the database elements are imported into the logic program as facts for computing secret answers (cf Rule 1 in Definition 34) This is inefficient, since we only require a subset of database facts In this section, we show how to obtain the appropriate data, by taking advantage of all the bult-in predicates such as $=,>,<$ in the query. The following example will illustrate the idea

Example 5.8. Consıder the database instance $D=\{P(1,5), P(1,3), S(1,2)\}$, a set $\mathcal{V}^{s}$ of secrecy views. $\left\{V_{s}(x) \leftarrow P(x, y), y<6, V_{s}(x) \leftarrow S(x, y)\right\}$, and the conjunctive query $\mathcal{Q} A n s(x) \leftarrow P(x, y), y=3$ with a built-ın predicate $y=3$ According to Definition 36, the revelent predicate for this query $\mathcal{Q}$ is $P$ Therefore, we do not need to import any data for the database predicate (or relation) $S$. Furthermore, only data need to be imported into the secrecy program is $P(1,3)$ instead of $P(1,5)$ and $P(1,3)$. This makes sense, since $P(1,5)$ can never be an answer to the query $\mathcal{Q}$, no matter whether it is defined in secrecy views or not

Algorithm 1 brings built-1n conditions in the query to the relevant database predicates to retrieve appropriate data facts This makes sense, since the bult-ins in the query would be used to restrict the tuples involved in the computation of the query

Example 5.9. (example 5.8 continued) The query $\mathcal{Q}$ contains a bult-ins $y=3$. The 


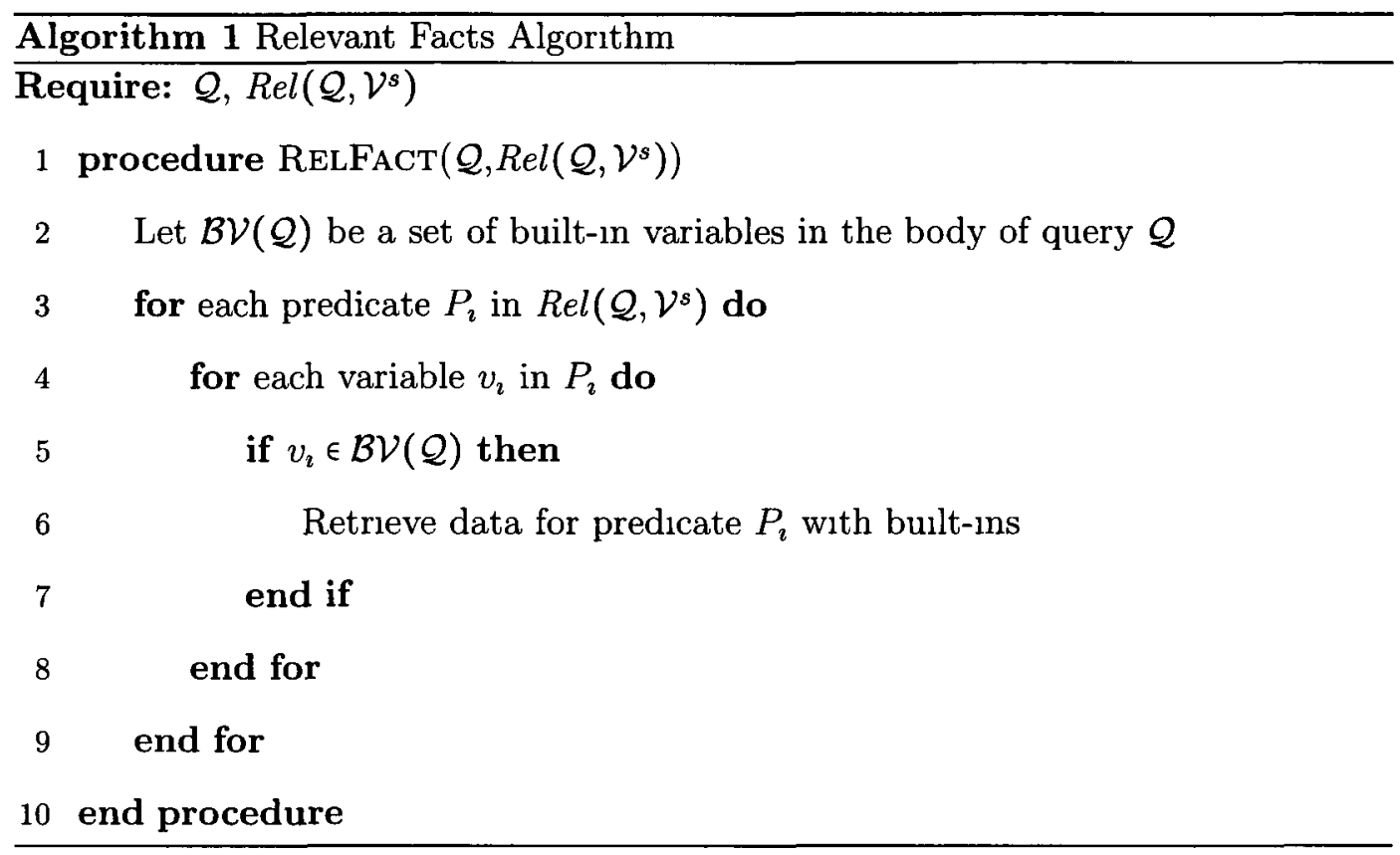

relevant predicate $P$ contains the variable $y$ We get the following query statement for the relevant predicate $P$ to retrieve relevant data facts:

SELECT * DISTINCT

FROM P

WHERE $\mathrm{Y}=3$

When evaluating secrecy and query programs in DLV, this query statement can be used to import data facts, residing in database systems into DLV (cf Section 61 for details)

\subsection{Summary}

In this chapter we have introduced disjunctive $\log _{1}$ programs with stable model semantıcs to specify the secrecy instances. These programs can be used to compute secret answers. 
We also presented a methodology to secrecy programs to optımıze the evaluation of programs. Optimization is achieved in two steps In the first step, the relevant database predicates are captured to remove the rules (and database facts) from the secrecy programs, as shown by Example 5.7. This is done by analyzing the relationship between the database predicates in queries and secrecy views, by meanings of dependency graph in Definition 35. As a result, we obtain reduced secrecy programs, 1 e. programs that consider the relevant predicates to compute a query. The second step in our optimization is to apply the bult-in conditions from the query to the relevant database predicates to retrieve the appropriate data. In this manner, only the set of data facts for the query is imported into the reasoning system

The optimization reduces the amount of data involved in the computation of queries In Chapter 6, we report experimental results that show that optimized programs are more efficient to compute secret answers for queries than the straightforward evaluation of programs 


\section{Chapter 6}

\section{Experiments}

In this chapter, we present experimental results about the computation of secret answers to queries. These queries are representative of the type of queries in our research. The experiments also show query answering with and without the optimizations steps introduced in Section 5.1

\subsection{Preliminaries}

Secret answers to queries can be computed by evaluatıng queries against the secrecy programs For this purpose we use the DLV system, that implements the stable model semantıcs of disjunctive logic programs [20,30] In this section, we will briefly the use of the $\operatorname{DLV}^{D B}$ system to compute secret answers $\operatorname{DLV}^{D B}[39,31]$, an extension of the DLV system, that provides flexible and easy interfaces with external commercial database management systems via ODBC (Open Database Connectivity)

Figure 61 illustrates the general architecture used in query evaluation. The data is stored in a relational DBMS, in our case, IBM DB2 The input for query evaluation includes the following a first-order conjunctive query $\mathcal{Q}$, a database instance $D$, and a set $\mathcal{V}^{s}$ of secrecy views. The only output of this architecture is the set of secret answers to the input query $\mathcal{Q}$ with respect to $\mathcal{V}^{s}$ We will use the example below to illustrate the application of the DLV system to the computation of secret query answers 


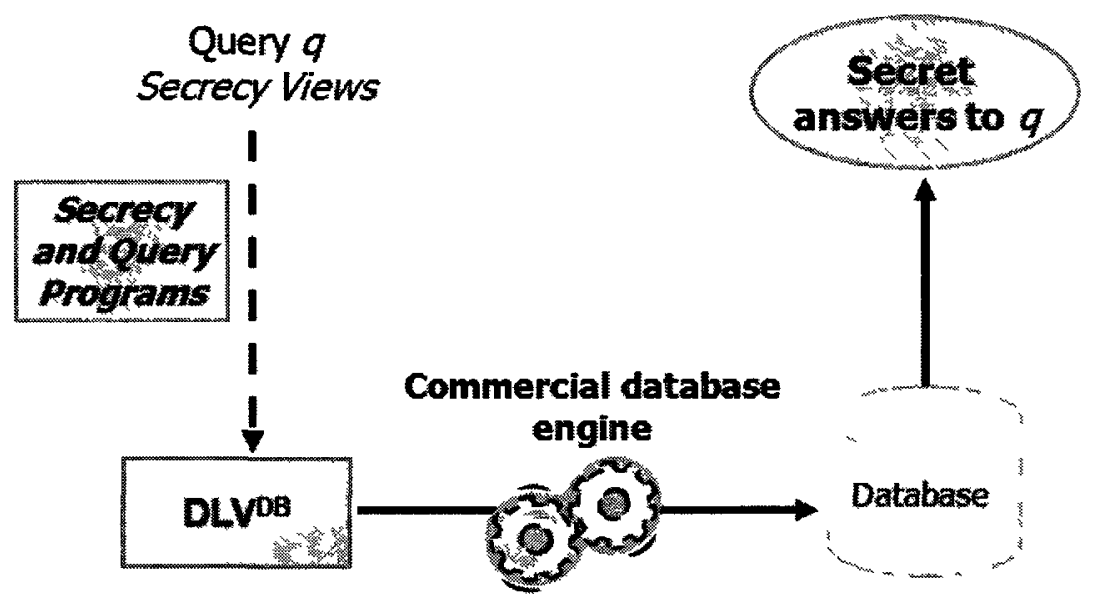

Figure 61 Architecture of Query Evaluation

Example 6.1. Consider the database instance $D=\{R(a, b), S(b), R(b, c)\}$, and a secrecy view $V_{s} V_{s}(x) \leftarrow R(x, y), S(y) \quad D$ contains secret information $V_{s}(a)$ wrt $V_{s}$ There are three secrecy instances

$$
\begin{aligned}
& D_{1}=\{R(a, n u l l), R(b, c), S(b)\} \\
& D_{2}=\{R(a . b), R(b, c), S(n u l l)\} \\
& D_{3}=\{R(n u l l, b), R(b, c) . S(b)\}
\end{aligned}
$$

For query $\mathcal{Q}: A n s(x) \leftarrow R(x . y)$, the secret answer is $(b)$.

First, the secrecy program $\Pi\left(D, \mathcal{V}^{s}\right)$ needs to be constructed wrt the given database instance $D$ and the set $\mathcal{V}^{s}$ of secrecy views $V_{s}$. Here, the facts of the program are not imported from the database directly. Instead, some suitable sentences are included into the secrecy program which can be interpreted by $\mathrm{DLV}^{D B}$

The secrecy program contains, for each extensional predicate $P$, the following Import sentence:

$$
\text { \# mmport (dbName,dbUser, dbPassword, "SELECT * FROM P", P, typeConv), }
$$

where $d b N a m e$ is the database name, dbPasswrod is the user password, and $d b U s e r$ 
1s the user of the database. $P$ defines the name of the predicate that will be used in the program

Since DLV ${ }^{D B}$ supports only unsigned integer and constant data types, typeConv specifies the conversion for mapping DBMS data types to $\mathrm{DLV}^{D B}$ data types for each column. The typeConv parameter is a string with the following syntax: type Conv [, Conv], where type: is a string constant and Conv is one of several conversion types

- U_Int: the column is converted to an unsigned integer,

- Const the column is converted to a string without quotes,

- Q_Const: the column is converted to a string with quotes

The number of the entries in the conversion list has to match the number of columns in the selected table

Example 6.2. Assume the following table definition:

CREATE TABLE p ( X varchar(10))

We insert two tuples in the table $p$ using the following SQL statement*

INSERT INTO $\mathrm{p}$

VALUES ('a'), ('b')

Let us now use import the table $p$ into the $\mathrm{DLV}^{D B}$ syetem. Let the file importonly $d l v$ contains just a single \#import statement

$$
\text { \# }
$$

Invoking $\mathrm{DLV}^{D B}$ with this file yields

$$
\begin{aligned}
& d l-\text { silent ımportonly.dlv } \\
& \{P(\text { (a") }), P(\text { " } b ")\}
\end{aligned}
$$


The varchar values converted as strings with quotes, which are constants in the $\mathrm{DLV}^{D B}$ system.

As a result, the database facts stored in the database $d b N a m e$ will be imported into the reasoning system $\mathrm{DLV}^{D B}$, where the secrecy program is run Now we describe how DLV ${ }^{D B}$ interacts with DBMS.

Program 1 is the secrecy program for the database instance $D$ and the secrecy view in Example 61 We assume the following database parameters

- Database name test

- Database user. db2admin

- Database password test

\section{Program 1.}

$$
\begin{aligned}
& \text { \#ımport(test, "db2admın", "test", "SELECT * FROM R", } \\
& R 1 \text {, type : Q_Const.Q_Const) } \\
& \text { \#import (test, "db2admın", "test", "SELECT * FROM S",S1, type : Q_Const). } \\
& R\left(x . y, \mathbf{t}_{\mathbf{d}}\right):-R 1(x, y) \quad S_{-}\left(y, \mathbf{t}_{\mathbf{d}}\right):-S 1(y) \\
& R\left(\text { ("null", } y, \mathbf{a}_{\mathbf{u}}\right) \vee R_{-}\left(x, \text { "null", } \mathbf{a}_{\mathbf{u}}\right) \vee S_{-}\left(" n u l l ", \mathbf{a}_{\mathbf{u}}\right) \\
& :-R(x, y, \mathbf{t}), S_{-}(y, \mathbf{t}), y \neq \text { "null”, aux }(x) \\
& \operatorname{aux}(x):-R(x, y, \mathbf{t}), S_{-}(y, \mathbf{t}), y \neq \text { "null", } x \neq \text { "null" } \\
& R\left(x, y, \mathbf{b}_{\mathbf{u}}\right):-R(x, y, \mathbf{t}), S_{-}(y, \mathbf{t}), R\left(\text { "null", } y, \mathbf{a}_{\mathbf{u}}\right), y \neq \text { "null", aux }(x), x \neq \text { "null" } \\
& R\left(x y, \mathbf{b}_{\mathbf{u}}\right):-R(x, y, \mathbf{t}), S_{-}(y, \mathbf{t}), R\left(x, " n u l l ", \mathbf{a}_{\mathbf{u}}\right), y \neq \text { "null", aux }(x) . \\
& S_{-}\left(y, \mathbf{b}_{\mathbf{u}}\right):-R(x, y, \mathbf{t}), S_{-}(y, \mathbf{t}), S_{-}\left(" n u l l ", \mathbf{a}_{\mathbf{u}}\right), y \neq \text { "null", aux }(x) \\
& R(x, y, \mathbf{t}):-R\left(x, y, \mathbf{t}_{\mathbf{d}}\right) . \quad R(x, y, \mathbf{t}):-R\left(x, y, \mathbf{a}_{\mathbf{u}}\right) \\
& S_{-}(x, \mathbf{t}):-S_{-}\left(y \cdot \mathbf{t}_{\mathbf{d}}\right) \quad S_{-}(y, \mathbf{t}):-S_{-}\left(y, \mathbf{a}_{\mathbf{u}}\right) \text {. } \\
& R(x, y, \mathbf{s}):-R_{-}(x, y, \mathbf{t}), \operatorname{not} R_{-}\left(x, y, \mathbf{b}_{\mathbf{u}}\right)
\end{aligned}
$$


$S_{-}(y, \mathbf{s}):-S_{-}(y, \mathbf{t}), \operatorname{not} S_{-}\left(y, \mathbf{b}_{\mathbf{u}}\right)$.

Secrecy programs should be stored for other queries. This is because the secrecy programs just depend on the given set of secrecy views and database relations, which are independent from the particular query. Therefore, they can be used for any other conjunctive query to compute secret answers In fact, secrecy programs need to be rewritten only when the database schema or secrecy views are changed

After having the secrecy program for the given database $D$, the secret answers to the query $\mathcal{Q}$ posed to $D$ can be computed by running the query program in combination with the secrecy program The query program for the query $\mathcal{Q}$ in Example 61 is the following

Program 2. The query program for the query $\mathcal{Q}$

$\operatorname{Ans}(X):-R(X, Y, \mathbf{s})$

$\operatorname{Ans}(X)$ ?

As a reminder, we need to emphasize here that the query to be run has to be transformed into one that treats the nulls as any other constant

Finally, the combıned program, stored in a text file named ex dlv, is run in DLV ${ }^{D B}$ under skeptical (cautious) reasoning to compute secret answers as follows

$$
\begin{aligned}
& d l \text { exe-silent-cautrous ex } d l v \\
& \text { "b" }
\end{aligned}
$$

For Program 1, DLV ${ }^{D B}$ returns $(b)$ as the secret answer to user, as we expected Of course, we could use the optimization steps presented in this research to improve efficiency. The next section, we will look at the execution time on computation of secret answers to queries with and without optımization. 


\subsection{Experimental Setup}

Serval experiments on computation of secret answers to querıes were run on DLV system, performed by a HP PC with Inter(R) Core(TM)2 CPU and 2GB RAM using Windows 7 operating system. The database instance was stored on the IBM DB2 Professional Database Server Edition V9.7. All the programs were run in the version of $\mathrm{DLV}^{D B}$ for Windows released on September 20th, 2007

For the experiments we consider the database instance $D$, with 300 tuples for an company database with following relations and the set of secrecy views:

- Employee $(E N O, E N A M E, S A L A R Y, D N O)$ It stores the employee number, name, salary, and department number of employees.

- Department $(D N O, D N A M E)$ It stores the department number, and name of departments

- Project( $P N O, P N A M E, A I D)$. It stores the project's numbers, names, and addresses.

- Address (AID, CITY, STATE, COUNTRY) It stores address information wrt city, state and country.

The set $\mathcal{V}^{s}$ of secrecy views is given by

$$
\begin{aligned}
& V_{s}(\text { dno, dname }) \leftarrow \text { Department }(\text { dno, dname }), \text { dname = "operations". } \\
& V_{s}(\text { eno, ename }) \leftarrow \text { Employee }(\text { eno, ename, salary, dno }), \text { salary }<30,000 \\
& V_{s}(\text { pno, pname }) \leftarrow \operatorname{Project}(\text { pno, pname }, \text { ald }), \\
& \text { Address(azd, cuty, state, country), cıty = "ott" }
\end{aligned}
$$

Assuming the database name company, the database user ad2admin, and the password test, the secrecy program for the database instance $D$ contains the rules in Program 3 


\section{Program 3.}

$$
\begin{aligned}
& \text { \#import(company, "db2admın", "test", "SELECT * FROM Employee", Emp, } \\
& \text { type.Q_Const,Q_Const, } \\
& \text { Q_Const,Q_Const). } \\
& \text { \#import(company, "db2admın", "test", "SELECT * FROM Department", Dept, } \\
& \text { type :Q_Const,Q_Const). } \\
& \text { \#import (company, "db2admin", "test", "SELECT * FROM Project", Proj, } \\
& \text { type :Q_Const,Q_Const,Q_Const). } \\
& \text { \#import (company, "db2admin", "test", "SELECT * FROM Address", Addr, } \\
& \text { type } \cdot Q_{-} \text {Const, Q_Const, } \\
& \text { Q_Const,Q_Const). } \\
& \text { Department_(dno,dame, } \left.\mathbf{t}_{\mathbf{d}}\right):-\operatorname{Dept}(\text { dno,dname }) \\
& \text { Department_(dno, "null", } \left.\left.\mathbf{a}_{\mathbf{u}}\right): \text {-Department_(dno, "Operatıons", } \mathbf{t}\right) \\
& \text { Department_(dno, "Operatıons", } \left.\left.\mathbf{b}_{\mathbf{u}}\right):- \text { Department_(dno, "Operatıons", } \mathbf{t}\right) \text {, } \\
& \text { Department_(dno, "null", } \left.\mathbf{a}_{\mathbf{u}}\right) \text {. }
\end{aligned}
$$

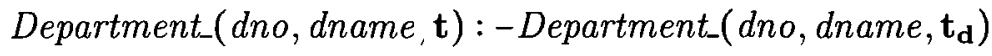

$$
\begin{aligned}
& \text { Department_(dno,dname, } \left.\mathbf{t}):- \text { Department_(dno,dname, } \mathbf{a}_{\mathbf{u}}\right) \text {. }
\end{aligned}
$$

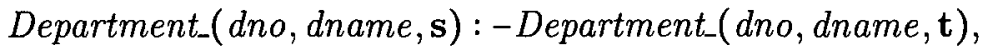

$$
\begin{aligned}
& \text { not Department_(dno, dname, } \left.\mathbf{b}_{\mathbf{u}}\right) \text {. } \\
& \text { Employee_(eno, ename, salary, dno, } \left.\mathbf{t}_{\mathbf{d}}\right):-\operatorname{Emp}(\text { eno, ename, salary, dno }) \text {. }
\end{aligned}
$$

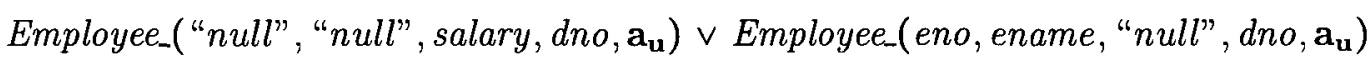

$$
\begin{aligned}
& \text { :-Employee_(eno, ename, salary, dno, t), } \\
& \text { salary < "30000", salary } \neq \text { "null", } \\
& \text { auxEmp (eno, ename) } \\
& \text { auxEmp (eno, ename) :-Employee_(eno, ename, salary, dno, } \mathbf{t}) \text {, } \\
& \text { salary < "30000", salary } \neq \text { "null", } \\
& \text { eno } \neq \text { "null". }
\end{aligned}
$$




$$
\begin{aligned}
& \text { auxEmp }(\text { eno, ename }):- \text { Employee }(\text { eno, ename, salary }, \text { dno, } \mathbf{t}), \\
& \text { salary }< \text { "30000", salary } \neq \text { "null", } \\
& \text { ename } \neq \text { "null". }
\end{aligned}
$$

Employee_(eno, ename, salary, dno, $\left.\mathbf{b}_{\mathbf{u}}\right):-$ Employee_(eno, ename, salary, dno, $\left.\mathbf{t}\right)$, salary < "30000", salary $\neq$ "null", auxEmp (eno, ename),

Employee_(eno, ename, "null", dno, $\left.\mathbf{a}_{\mathbf{u}}\right)$

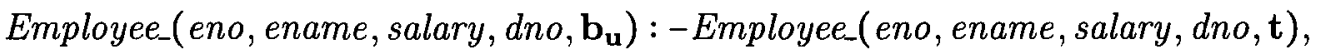

$$
\begin{aligned}
& \text { salary< "30000", salary } \neq \text { "null", } \\
& \text { auxEmp (eno, ename }), \\
& \text { Employee("null", "null", salary, dno, } \left.\mathbf{a}_{\mathbf{u}}\right), \\
& \text { eno } \neq \text { "null", ename } \neq \text { "null". }
\end{aligned}
$$

Employee_(eno, ename, salary, dno, $\mathbf{t}):-$ Employee $\left(\right.$ eno, ename, salary, dno, $\left.\mathbf{t}_{\mathbf{d}}\right)$.

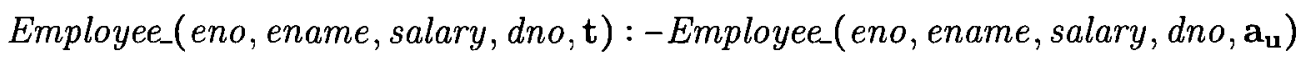

Employee_(eno, ename, salary,dno, s) :-Employee_(eno, ename, salary, dno,t), not Employee_(eno, ename, salary, dno, $\left.\mathbf{b}_{\mathbf{u}}\right)$.

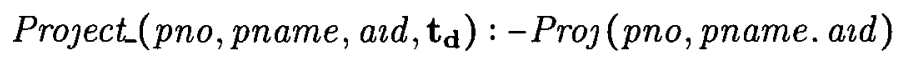

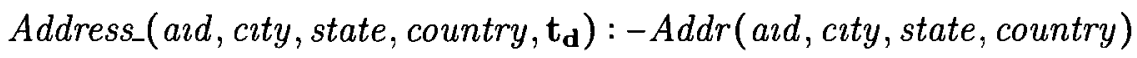

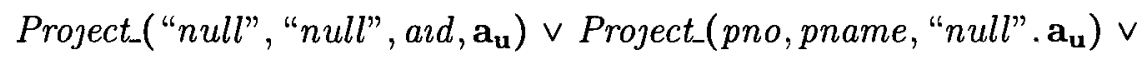

Address_("null", "ott", state, country, $\left.\left.\mathbf{a}_{\mathbf{u}}\right) \vee \operatorname{Address\_ (aud,~"null",~state,~country,~} \mathbf{a}_{\mathbf{u}}\right)$

$$
\text { :-Project_(pno, pname, ard.t), }
$$

Address_(aıd, "ott", state. country.t),

$$
\text { aıd } \neq \text { "null", auxProj(pno,pname) }
$$

$$
\begin{aligned}
& \text { auxProj(pno, pname) :-Project_(pno, pname, ard.t), } \\
& \text { Address_(aid, "ott", state. country.t), } \\
& \text { aid } \neq \text { "null", pno } \neq \text { "null" }
\end{aligned}
$$$$
\text { auxProj (pno, pname) :-Project_(pno.pname, ard.t), }
$$ 
Address_(aud, "ott", state, country.t), aid $\neq$ "null", pname $\neq$ "null"

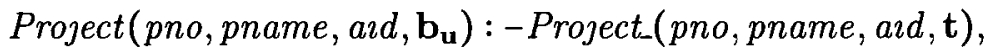
Address_(azd, "ott", state, country.t), ald $\neq$ "null", auxProj(pno, pname).

Project_(pno.pname, "null", $\left.\mathbf{a}_{\mathbf{u}}\right)$

Project_(pno, pname, ald, $\left.\mathbf{b}_{\mathbf{u}}\right):-$ Project_(pno. pname, ald, $\left.\mathbf{t}\right)$, Address_(aud, "ott", state, country.t), ald $\neq$ "null", auxProj(pno, pname), Project_("null", "null", aid, $\left.\mathbf{a}_{\mathbf{u}}\right)$, pno $\neq$ "null", pname $\neq$ "null"

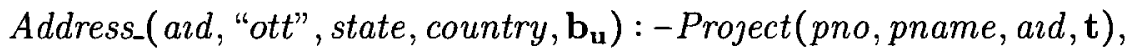
Address(aid, "ott", state, country $\mathrm{t}$ ), ald $\neq$ "null", auxProj(pno, pname), Address("null", "ott", state, country, $\mathbf{a}_{\mathbf{u}}$ )

Address_(aid. "ott", state, country, $\left.\mathbf{b}_{\mathbf{u}}\right):$-Project_(pno, pname, ald, $\left.\mathbf{t}\right)$, Address_(aud, "ott", state, country.t) åd $\neq$ "null", auxProj(pno, pname), Address_(aid, "null", state, country, $\left.\mathbf{a}_{\mathbf{u}}\right)$.

Project_(pno, pname, and, $\mathbf{t}):-\operatorname{Project}\left(\right.$ pno, pname, ald, $\left.\mathbf{t}_{\mathbf{d}}\right)$ Project_(pno, pname, ard.t) :-Project_(pno pname, and, $\left.\mathbf{a}_{\mathbf{u}}\right)$.

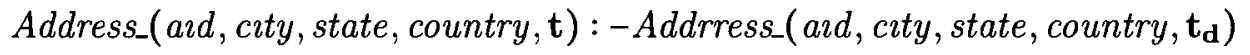

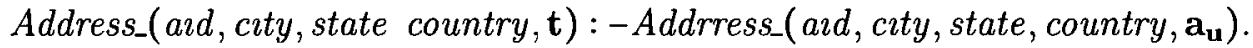

Project_(pno, pname, aid, s) :-Project_(pno.pname, ald, t), not Project_(pno. pname, ald. $\left.\mathbf{b}_{\mathbf{u}}\right)$.

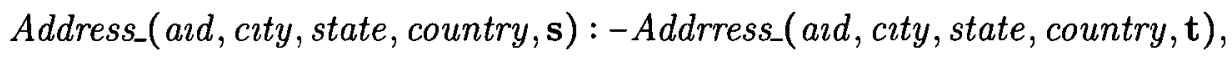
not Addrress_(aid, city, state, country, $\left.\mathbf{b}_{\mathbf{u}}\right)$. 
Notice that when Program 3 is evaluated in DLV, the whole company database stored in the DBMS will be imported into DLV, which is inefficient. Therefore, we need to reduce as much as possible the interaction between the DBMS, where the data resides, and the DLV system, where the secrecy program is evaluated. As indicated above, our optimized methodology can generate programs for queries that will import only a subset of the database. We illustrate effectiveness by running three conjunctive queries that are representative of the type of queries in this research

\subsubsection{Test Case1}

A conjunctive query $\mathcal{Q}_{1}$ that asks for employee's number and name if his or her salary is less than 40000 :

$$
\text { Ans }(\text { eno, name }) \leftarrow \text { Employee }(\text { eno.name. salary, deptno }), \text { salay }<40000
$$

This query is open, 1 e with free variables and bult-ins

Program 4 is the query program for query $\mathcal{Q}_{\mathbf{1}}$. The secrecy program listed in Program 3 together with this query program can be evaluated using the DLV system to obtain secret answers Of course, this is a naive method for computing answers, which imports all the data from the database and using the rules for all secrecy view definitıons.

\section{Program 4.}

$$
\begin{gathered}
\text { Ans }(\text { eno, ename }):- \text { Employee }(\text { eno, ename. salary, dno }, \mathbf{s}), \\
\text { salary } \neq \text { "null", salary }<40000 .
\end{gathered}
$$

For the query $\mathcal{Q}_{1}$, the programs can be optımized by using the relevant predicates and facts (cf Section 51 ). Here, the relevant predicate for query $\mathcal{Q}_{1}$ wrt the set $\mathcal{V}^{s}$ of secrecy views is Employee. According to Algorithm 1, the bullt-1n predicate salary $<40000$ in query $\mathcal{Q}_{1}$ can be applied to the relevant predicate Employee The 
following is the import sentence in the optimized program which brings only relevant data facts and predicates into DLV.

$$
\begin{array}{r}
\text { \#import(company, "db2admın", "test", "SELECT * FROM Employee } \\
\text { WHERE Salary }<40000 ", E m p, \\
\text { type }: \text { Q_Const, Q_Const,Q_Const,Q_Const) }
\end{array}
$$

Program 7 in Appendix A lists all the rules of the optımized program for the query $\mathcal{Q}_{1}$.

\subsubsection{Test Case2}

The second query $\mathcal{Q}_{2}$ asks for employee's name, who works in "sales" department.

$$
\begin{aligned}
\text { Ans }(\text { name }) \leftarrow \text { Employee }(\text { eno, name, salary, dno }), & \\
& \text { Department }(\text { dno, dname }), \text { dname = "sales" }
\end{aligned}
$$

This conjunctıve query is open with joins and bult-ins This type of queries is widely used in practice The query program for $\mathcal{Q}_{2}$ shows as follows:

\section{Program 5.}

$$
\begin{aligned}
& \text { Ans }(\text { ename }):- \text { Employee }(\text { eno, ename, salary, dno, }), \\
& \text { Department }(\text { dno, dname }, \mathbf{s}), \text { dname }=\text { "sales", } \\
& \text { dname } \neq \text { "null",dno } \neq \text { "null" }
\end{aligned}
$$

This query program plus Program 3 can compute secret answers for the conjunctive query $\mathcal{Q}_{2}$. Program 8 in Appendix A is the optimized program for the secrecy program in 3 , and the query program in 5 . Notice that in Program 8 only the relevant portion of the database is imported into DLV This is, only tuples for relations Employee, and Department are imported into DLV The following are two import sentences will be 
used in the optimized program.

$$
\begin{aligned}
& \text { \# ımport(company, "db2admın", "test", "SELECT * FROM Employee", } \\
& \text { type : Q_Const, Q_Const, Q_Const, Q_Const). } \\
& \text { \#import(company, "db2admın", "test", "SELECT * FROM Department } \\
& \text { WHERE dname = 'sales'", Emp, type : Q_Const, Q_Const). }
\end{aligned}
$$

\subsubsection{Test Case3}

Consider the following boolean query $\mathcal{Q}_{3}$ :

$$
\text { Ans } \leftarrow \text { Department }(\text { dno, dname }), \text { dno = "101", dname = "sales". }
$$

The query program to compute secret answers for $\mathcal{Q}_{3}$ shows as follows

\section{Program 6.}

$$
\begin{aligned}
& \text { Ans:-Department }(\text { dno,dname }, \mathbf{s}), \text { dno }=\text { "101", dname }=\text { "sales", } \\
& \text { dno } \neq \text { "null", dname } \neq \text { "null" }
\end{aligned}
$$

The optımized program in 9 only imports one tuple Department $(101$, sales $)$ into DLV for this boolean query. The import sentences in the Program 9 is.

$$
\begin{array}{r}
\text { \#import(company, "db2admin", "test", "SELECT * FROM Department } \\
\text { WHERE Name = 'sales' AND Depo = ' } 101 \text { ", } \\
\text { Dept, type }: \text { Q_Const, Q_Const). }
\end{array}
$$

\subsection{Results}

Figure 6.2, 63 and 6.4 illustrate the execution times of the three queries on the database instance $D$. 


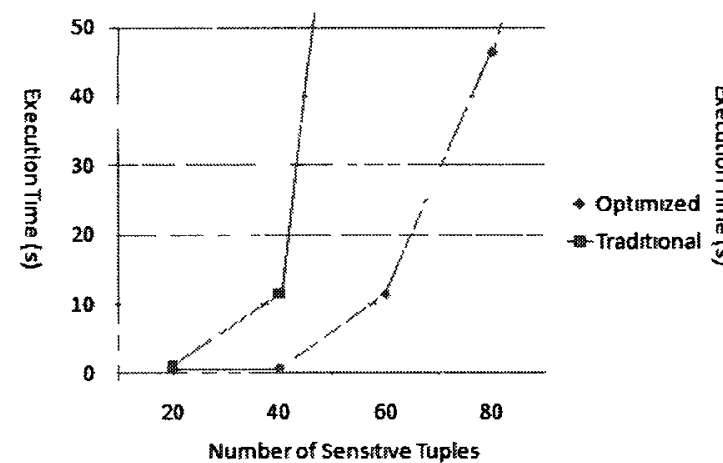

Figure 62 Execution Time for $\mathcal{Q}_{1}$

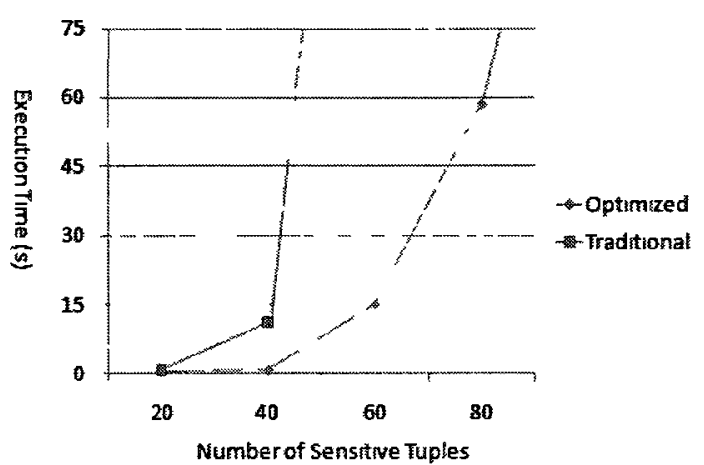

Figure 6 3: Execution Time for $\mathcal{Q}_{2}$

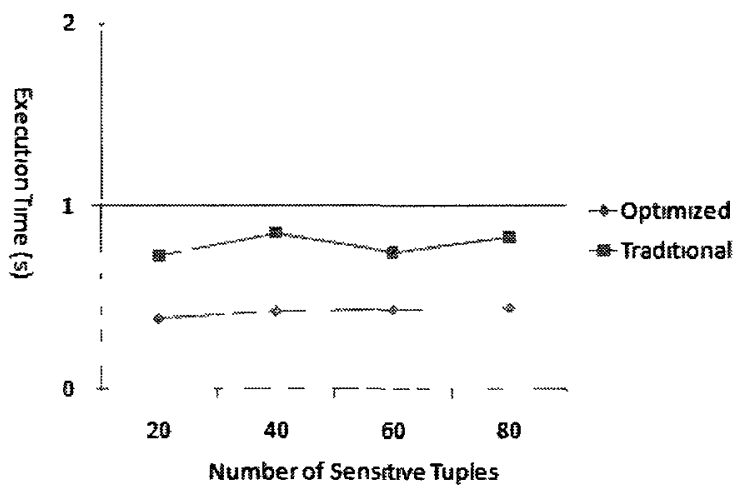

Figure 64 Execution Time for $\mathcal{Q}_{3}$

We can see that, the optimized programs are faster to compute secret answers to queries than the straightforward evaluation of program. This is because they capture the relevant predicates to compute query As a result, only the rules, and database facts, that are relevant to compute a query are kept in them Therefore, the data flow between the DLV system and the database is reduced

Based on the experiments performed, we conclude that the computation of secret answers based on logic programs is viable, especially for the a ground (or partiallyground) query. This makes sense, bult-ins in queries would be used to restrict the tuples involved in the computation of the answers

Optimizations on the process of retrieving answers have been widely studied in 
the literature The magic sets (MS) method $[21,6,34]$ is one of the best known techniques for optimizing query answering over logic programs The idea behınd magic sets is to determine sets of facts that are relevant to the query, and then use these facts to reduce the amount of data used in query evaluation. In $[13,14]$, the MS technıque for repair programs for consıstent query answerıng in DLV is applied In [13], experiments on the computation of consistent answers to queries using MS technique are presented. From those experiments, it is possible to conclude that MS technique has an excellent performance on query evaluation It is a future work to apply MS technique to our secrecy program to further improve efficiency. 


\section{Chapter 7}

\section{Discussion}

\subsection{Connection with CQA}

Consider a database instance $D$ that falls to satısfy a given set of integrity constraints $I C$ It still contains useful and correct information The area of consistent query answering (CQA) [8] has to do with. (a) Characterizing the information in $D$ that is still semantically correct wrt $I C$, and (b) Characterizing, and computing, in particular, the semantically correct, i e consistent, answers to a query $\mathcal{Q}$ from $D$ wrt $I C$. The first goal is achieved by proposing a repair semantıcs, i e a class of alternative instances to $D$ that are consistent wrt $I C$ and minimally depart from $D$ The consistent information in $D$ is the one that is invariant under all the repairs in the class. This applies in particular to the consistent answers They should hold in every minimally repaired instance

There are some connections between CQA and our treatment of privacy preserving query answerıng. Notıce that every view definition of the form (212) can be seen as an integrity constraint expressed in the FO language $L\left(\Sigma \cup\left\{V_{s}\right\}\right)$.

$$
\forall \bar{x}\left(V_{s}(\bar{x}) \longleftrightarrow \exists \bar{y}\left(R_{1}\left(\bar{x}_{1}\right) \wedge \cdots \wedge R_{n}\left(\bar{x}_{n}\right) \wedge \varphi\right)\right)
$$

with $\bar{y}=\left(\cup \bar{x}_{\imath}\right) \backslash \bar{x}$ From this perspective, maintaining the view defined by (7 1) (i.e. synchronized with the base relations) [23] becomes a problem of database maintenance, i e. maintenance of the consistency of the database wrt (7 1) seen as an IC. This also works in the other direction since every IC can be associated to a violation view, which has to stay empty for the IC to stay satisfied. 
Actually, we want more than to maintain the view defined in (7 1) We want it to be empty or returning only tuples with null values In consequence, we have to impose the following ICs on $D$, which are obtained from the RHS of (71): If $\bar{x}$ is $x^{1}, ., x^{k}$, then for $1 \leq \imath \leq k$,

$$
\forall \bar{x} \bar{y} \neg\left(R_{1}\left(\bar{x}_{1}\right) \wedge \cdots \wedge R_{n}\left(\bar{x}_{n}\right) \wedge \varphi \wedge x^{2} \neq n u l l\right)
$$

That is, from each view definition (7 1) we obtain $k$ denıal constraints, 1 e prohibited conjunctions of (positive) database atoms and bult-ıns, which have been investıgated in CQA $[16,8]$. In consequence, the secrecy instances correspond to the repairs of $D$ wrt the set $I C$ of all the ICs of the form (72), obtained from each of the secrecy view definitions These repairs are defined according to the null-based semantics introduced in Sectıon 4.1, i e $\leq_{D}$-mınimality, which was investıgated in $[12,11]$. This reduction allows us to profit from exısting results for CQA

Example 7.1. The secrecy view defined by $V_{s}(x, z) \leftarrow P(x, y), R(y, z)$ gives rise to the following denial constraints:

$$
\begin{aligned}
& \neg \exists x y z(P(x, y) \wedge R(y, z) \wedge x \neq n u l l), \\
& \neg \exists x y z(P(x, y) \wedge R(y, z) \wedge z \neq n u l l),
\end{aligned}
$$

The initıal instance $D$ has to be minimally reparred in order to satısfy them

\subsection{Connection with Data Cleaning}

A database instance may contain several tuples in it that refer to the same external entity that is being modeled by the database This problem could be caused by errors in data, by data coming from different sources that use different formats, etc. 
In this sense, the database is considered to contain duplicate data. These duplicates could compromise secrecy, as lllustrated in the following example

Example 7.2. Consider a database $D$ that includes the following relation Student, with attributes Name, ID, DOB, Gender, and AvgMark (we use an extra column to denote the tuple):

Table 7 1: Student Table

\begin{tabular}{|c|c|c|c|c||c|}
\hline Name & $I D$ & DOB & Gender & AvgMark & \\
\hline J. Smith & 001 & 880801 & $M$ & 60 & $t 1$ \\
John Smith & 001 & 880801 & $M$ & 60 & $t 2$ \\
\hline
\end{tabular}

Assume the following secrecy view defined on the relation Student to protect John Smıth's average mark

$$
V_{s}(v) \leftarrow \operatorname{Studnet}(x, y, z, u, v), x={ }^{'} J o h n S m \imath t h '
$$

According to the secrecy semantıcs proposed in Chapter 4, we obtain the following two secrecy instances

\begin{tabular}{|c|c|c|c|c|}
\hline Name & $I D$ & DOB & Gender & AvgMark \\
\hline J. Smith & 001 & 880801 & $M$ & 60 \\
John Smıth & 001 & 880801 & $M$ & null \\
\hline
\end{tabular}

\begin{tabular}{|c|c|c|c|c|}
\hline Name & $I D$ & DOB & Gender & AvgMark \\
\hline$J$ Smith & 001 & 880801 & $M$ & 60 \\
null & 001 & 880801 & $M$ & 60 \\
\hline
\end{tabular}

Suppose the user issues the following query:

$$
\operatorname{Ans}(x, y, z, u, v) \leftarrow \operatorname{Student}(x, y, z, u, v)
$$

Query (7 4) is evaluated against the above set of secrecy instances, and it will get tuples $t_{1}=S t u \operatorname{dent}(J$ Smith,001,880801, $M$, null $)$, and $t_{3}=\operatorname{Student}(n u l l, 001,880801$, 
$M, n u l l)$ as secret answers. Actually, two tuples $t_{1}, t_{3}$ refer to the same student Even though the name of $t_{1}$ is not identical to the name of $t_{3}$, the same student id in the two tuples is sufficient to establish that the tuples refer to the same student. Thus, $t_{1}$ and $t_{3}$ are considered as duplicates. The user can take advantage of duplicates to infer sensitive information In our case, he can obtain John Smith's average mark 60 from $t_{1}$, which is sensitive information.

The above example shows that even if the data privacy implementation ensures that the query result contains only authorized information, it is possıble for users to gain access to sensitive informatıon based on duphcates, which brings the issue of duplicate detection, and data cleaning [36]

The Suspect Duplacate Processing (SDP) feature in IBM InfoSphere Master Data Management Server (MDM) provides a practical method of duplicate detection We could make use of this practical method to remove duplicates Before presenting the 1deas, we give a brief introduction to MDM

Master data [18] is the most valuable data that an organization owns, which represents core information about the business, such as customers, products, and accounts etc. Since it is highly valuable, all parts of an organization must agree on it In an ideal world, we expect a single place where all master data in an organization is stored and managed All updates should take place against this single copy of master data, and all the users of master data should also interact with this single copy. In this way, the organization can use the information in a consistent way

In fact, the goal of MDM is to enable this ideal state It provides a consistent way of using master data entities In particular, it has the SDP feature which provides a mechanısm to keep a single copy of customer data SDP involves two steps to examıning data to find duplicates in the relation. The first step is to select the match criteria and weights, and the second one is matching. 
In the rest of this section, we wll illustrate how to SDP find duplicate tuples in Student relation defined in Table 7.1. Although our illustration uses a relation about students, in general this approach is applicable to any relational database.

Not every data element is important from the suspect duplicate processing point of view. Therefore, data administrators of MDM need to identify the set of date elements that best help to uniquely identify the record in the relation according business requirements This set of data elements is known as critıcal data elements, which will be used to determine a match

Example 7.3. (example 72 contınued) Consıder the relation Student The same student $1 \mathrm{~d}$ in the two tuples is sufficient to establish that the tuples refer to the same student In addition, if for two tuples the values of name, date of birth, and gender are the same, then two tuples may be duplicates So, a set of critıcal data elements for the Student includes ID, Name, DOB, and Gender

After the critical data elements have been selected, weights need to be assigned to each critical element. This makes sense, since $I D$ should have a higher weight for matching than $D O B$. Assignment of weights is based on expert domain knowledge of the MDM data administrators. An example of a weight assignment for each critical data element in the Student relation is shown in the following table:

\begin{tabular}{|c|c|}
\hline Critical Data Element & Weight \\
\hline Gender & 1 \\
Data of Bırth & 2 \\
Name & 4 \\
ID & 8 \\
\hline
\end{tabular}

Based on the weight assignment, MDM DBA needs to define the Match Relevancy table shown in Table 7.2, in which defines a combinatıon of critıcal data elements that match, and shows what the score is for such a combination

Basically, match relevancy describes the critical data elements between two tuples 
Table 7 2. Match Relevancy

\begin{tabular}{|l|l|l|}
\hline Match_Relev & Name & Score \\
\hline 1 & All Elements Matched & 15 \\
2 & ID,Name,DOB & 14 \\
3 & ID,Name,Gender & 13 \\
4 & ID,DOB,Gender & 11 \\
. &. &. \\
\hline
\end{tabular}

that match, and the corresponding score is used to measure the quality of the match The score is calculated by sum of weights of critical data elements that match. For the two tuples in the Student table, values of $I D, D O B$, and Gender match The corresponding match relevancy score is 11 calculated by adding up the weights of $I D$, $D O B$, and Gender, as indicated in the fourth row in Table 7.2.

Similarity, MDM DBA also needs to define the Non-Match Relevancy table shown in Table 73 , which follows the same pattern as the Match Relevancy table

Table 73 Non-Match Relevancy

\begin{tabular}{|l|l|l|}
\hline None_Match_Relev & Name & Score \\
\hline 1 & All Elements Matched & 0 \\
2 & ID,Name,DOB & 1 \\
3 & ID,Name,Gender & 2 \\
4 & ID,DOB,Gender & 4 \\
.. & &.. \\
\hline
\end{tabular}

Non-Match relevancy describes the critical elements between two tuples that do not match, and the corresponding score is calculated simply by adding up the weights for the critical data elements that don't match. For the tuples in the Student table, since the values of Name do not match (values of $I D, D O B$ and Gender match), the non-match relevancy score is 4 , as indicated in the fourth row of Table 73

Finally, MDM DBA should define the Match Matrıx table shown in Table 7.4, which brings match/non-match relevancy scores for two tuples to determine duplicate. 
Table 7.4: Match Matrix

\begin{tabular}{|l|l|l|}
\hline Match_Relev & None_Match_Relev & Category \\
\hline 1 & 1 & Duplicate \\
2 & 2 & Duplicate \\
3 & 3 & Duplicate \\
4 & 4 & Duplicate \\
$\ldots$ & .. & . \\
\hline
\end{tabular}

For example, the fourth row in Table 74 indicates that match relevancy 4 (ID, $D O B$, and Gender match), and non-match relevancy 4 (Name dose not match) lead to duplicate. Our tuples in relation Student satisfy the fourth row of the Match Matrix table Therefore, we can conclude that those two tuples are duplicates, and we need to delete one tuple to eliminate duplicate

\subsection{Related Work}

Several models for data privacy and access control have been proposed in the recent past $[3,29,37,44,9,10]$, which aims to grant a partıcular user or group access to individual data items in a relation. In contrast, although the current SQL supports access control at the level of tables or columns, it does not provide any way to specify authorization to control which tuples can be accessed by which users.

Virtual Private Database (VPD) feature of Oracle's $9 \imath$ [3] supports fine-gramed access control by transparent query modification. The authorization policy is encoded into PL/SQL functıons defined for each relation, which are used to return where clause predicates to be appended to the user query before it is executed. The added predicates ensure that the user gets to see only those tuples in each table that he or she is authorized to see As noted in $[19,35]$, because of the lack of a formal mathematıcal basis for VPD, it is very difficult to define policy functıons corresponding to business requirements, especially authorization policies involving joins of tables Our 
framework provides a simple language that enables us to specify authorization policy.

Cell-level access control is described by LeFevre et al. [29] Their work focuses on ensuring limited data disclosure, based on the premise that data owners have control over who is allowed to see their personal data and for what purpose In their work, they introduce two models of cell-level limited disclosure enforcement table semantıcs and query semantıcs. The implementation of such models is based on query modificatıon technique.

Rizvı et al. [37] present two basic approaches to enforce fine-grained access control. The first approach is the Truman model and the second one is the Non-Truman model In the Truman model, the access control is defined by a collection of authorization views for each relation in the database. Each user has associated a set of authorization views When a user issues a query, the query is modified transparently by replacing each relation in the query by the corresponding authorization view associated with the user As Rizvi et al [37] point out, using a Truman model, the answers to queries may be misleading, or worse, incorrect

Non-Truman models, by contrast, prevent misleadıng answers Under the NonTruman model, a query that violates access control polices is simply rejected, rather than modified. Only valıd querıes, 1 e, queries could be rewritten using only the authorization views, are answered

Both approaches in [37] specify access control by means of positive authorizations, i e , they represent privileges granted to a user. However, the lack of a positıve authorization for a given user does not prevent this user from receiving this authorization [7] For example, the user can derive such authorization from its ancestor users. In contrast, our work makes sure that certain users are never allowed access to sensitive data

In $[9,10,42]$, they take a different approach to address privacy issues in incomplete 
propositional databases The approach, Control Query Evaluatıon (CQE), is policydriven, and aims to ensure confidentiality on the basis of a logical framework. A security policy specifies the facts that a certain user is not allowed to access For each query posed to the database by that user, it is checked whether the answer to that query would allow the user to infer any sensitive information If this is the case, the answer is distorted by either lying or refusal or combined lying and refusal. In [25], they extend CQE to restricted incomplete first-order logic databases. In that case, CQE is used through a transformations from the specification language to the corresponding propositional language.

This approach does not seem to be comparable to ours This approach has a very high complexity even with propositional logic. In addition, their work does not consider the possible presence of null in the database In contrast, we assume that the database may contains null and null is treated as NULL in the SQL standard. Furthermore, it is very natural to expect to obtain as many "useful" answers as possible while still protecting sensitive information However, there is no formal definition for "maximum" useful answers in their work Hence, it is impossible to prove the approach provide the best answers. In our work, the 1dea of "maximum" useful answers was captured by secrecy instances which minımızes the difference to the original database. 


\section{Chapter 8}

\section{Conclusions and Future Work}

\subsection{Conclusions}

In this work, we have developed a logical framework and a methodology to answer conjunctive queries that do not reveal secret information as specified by secrecy views We have concentrated on the case of conjunctive secrecy views and conjunctive queries, but it is possible to relax these restrictions We have assumed that the databases may contain nulls, and also nulls are used to protect secret information, by virtually updating with nulls some of the attribute values In each of the resulting alternative virtual instances, the secrecy views either become empty or contain tuples showing only null values The queries can be posed against any of these virtual instances or cautiously against all of them, simultaneously

The update semantics enforces (or captures) two natural requirements That the updates are based on null values, and that the updated instances stay close to the given instance. In this way, the query answers become implicitly maximally informative, while not revealıng the original contents of the secrecy views.

The null values are treated as in the SQL standard, which in our case is reconstructed in classical logic This logical reconstruction captures well the SQL "semantics" (which in not clear or complete in the standard), at least for the case of conjunctive queries (and some extensions thereof) This is the main reason for concentrating on this kind of queries and views In this case, queries, views and ICs can be syntactically transformed into new FO formulas for which the evaluation or verification can be done by treating nulls as any other constant. 
We introduced disjunctive logıc programs with stable model semantics to specify the secrecy instances These programs can be used to compute secret answers We also presented a optimization methodology, which captures the relevant database predicates and facts to compute a specific query.

\subsection{Future Work}

Our work leaves several open problems that are matter of ongoing and future research.

- Complexity issues have to be explored For example, of deciding whether or not a particular instance is a secrecy instance of an original instance Also of deciding if a tuple is a secret answer. The connection with CQA, where similar problems have been investigated, looks very promising in this regard

- Another problem is about query rewriting, i e about the possibility of rewriting the original query into a new FO query, in such a way that the new query, when answered by the given instance, returns the secret answers From the connection with CQA we can predict that this approach has limited applicability, but whenever possible, it should be used, for its simplicity and lower complexity.

- Another dimension of the problem consists in adding ICs to the schema If they are known to the user and also that they are satisfied by the database, then privacy could be compromised. Also the updates leading to the virtual updates should take these ICs into account. In this case, the defintion of secrecy instance should have the extra condition of satisfying the ICs, because ICs could be volated through the process of updating databases 


\section{Bibliography}

[1] SQL-2003 standard part 1 Framework (sql/framework). Avallable at http://www.wiscorp.com/SQLStandards.html

[2] SQL-2003 standard part 2: Foundation (sql/foundation). Available at: http //www.wiscorp com/SQLStandards html

[3] The virtual private database in oracle9ir2 Available at: http //otn oracle com/deploy/security/oracle9ir2/pdf/vpd9ır2twp pdf.

[4] S Abiteboul, R Hull, and V. Vianu. Foundations of Databases Addison-Wesley Longman Publıshing Co, Inc, Boston, MA, USA, 1st edition, 1995

[5] M Arenas, L Bertossi, and J Chomicki Consistent query answers in inconsistent databases In Proceedings of the 18th ACM SIGMOD-SIGACT-SIGART Symposium on Princıples of Database Systems, PODS '99, pages 68-79, New York, NY, USA, 1999 ACM.

[6] F Bancilhon, D Maier, Y. Sagiv, and J D Ullman Magic sets and other strange ways to implement logic programs (extended abstract) In Proceedings of the 5th ACM SIGACT-SIGMOD Symposium on Principles of Database Systems, PODS '86, pages 1-15, New York, NY, USA, 1986 ACM

[7] E Bertıno, P. Samaratı, and S Jajodia. An extended authorızation model for relational databases IEEE Trans on Knowl and Data Eng, 9 85-101, January 1997

[8] L. Bertossi Consistent query answering in databases SIGMOD Rec , 35 68-76, June 2006.

[9] J. Biskup and T Weibert. Confidentiality policies for controlled query evaluation. In Proceedings of the 21st Annual IFIP WG 113 Working Conference on Data and Applıcatıons Security, pages 1-13, Berlin, Heidelberg, 2007. Springer-Verlag.

[10] J Biskup and $\mathrm{T}$ Weibert. Keeping secrets in incomplete databases. Int. J Inf Secur., 7:199-217, May 2008.

[11] L. Bravo. Handling inconsistency in databases and data integration systems In PhD. Thesis. Carleton University, Department of Computer Science, 2007

[12] L Bravo and L. Bertossi. Semantically correct query answers in the presence of null values. In Proceedings of EDBT WS on Inconsistency and Incompleteness in Databases (IIDB 06), volume 5956 of $L N C S$, pages 230-247 Springer, 2006. 
[13] M Canıupan. Optımizing and implementing repair programs for consistent query answering in databases. In PhD Thesis Carleton University, Department of Computer Science, 2007

[14] M. Caniupan and L Bertossi Optimizing repair programs for consistent query answering. In Proceedings of the 25th International Conference on The Chllean Computer Scrence Socrety, pages 3-12, Washington, DC, USA, 2005. IEEE Computer Society

[15] M Caniupan, L Bertossi, and L. Bravo. Optimızing repair programs and their evaluation for consistent query answering in databases (extended version) In Proceedings of the 25th International Conference on The Chilean Computer Sclence Socrety, pages 3-12, Washington, DC, USA, 2005 IEEE Computer Soc1ety

[16] J. Chomicki and J. Marcinkowski. Mmimal-change integrity maintenance using tuple deletions. Inf Comput, 197 90-121, February 2005.

[17] E F Codd Extending the database relational model to capture more meaning ACM Trans Database Syst , 4 397-434, December 1979

[18] A Dreibelbis, E Hechler, I Milman, M Oberhofer, P. Run, and D Wolfson. Enterprise Master Data Management. An SOA Approach to Managing Core Information IBM Press, 2008

[19] S. Dwivedı and B. Menezes Database access control for e-business - a case study In Proceedings of the 11th International Conference on Management of Data, COMAD '05, pages 168-175, 2005

[20] T Eiter, W Faber, N Leone, and G. Pfeifer The diagnosis frontend of the dlv system AI Commun, 12 99-111, January 1999.

[21] W. Faber, G Greco, and N. Leone. Magic sets and their application to data integration. J Comput. Syst Scı., 73:584-609, June 2007

[22] G H Gessert Four valued logic for relational database systems. SIGMOD Rec., 19 29-35, March 1990

[23] A. Gupta and I S Mumick. Maintenance of materialized views problems, techniques, and applications. IEEE Data Engineering Bulletın, 12(2)·3-18, June 1995.

[24] T Imıliński and W Lipskı, Jr. Incomplete informatıon in relational databases $J$ ACM, 31·761-791, September 1984

[25] C T Joachim Biskup and L. Wiese. Towards controlled query evaluation for incomplete first-order databases In Proceedings of the 6th International Symposium on Foundations of Information and Knowledge Systems, volume 5956 of LNCS, pages 230-247 Springer, 2010. 
[26] R. Kocharekar Nulls in relational databases' revised. SIGMOD Rec , 18:68-73, March 1989.

[27] J. Lechtenborger. The impact of the constant complement approach towards view updating. In Proceedings of the 22nd ACM SIGMOD-SIGACT-SIGART Symposıum on Princıples of Database Systems, PODS '03, pages 49-55, New York, NY, USA, 2003. ACM.

[28] J. Lechtenborger and G. Vossen. On the computation of relational view complements ACM Trans. Database Syst., 28 175-208, June 2003.

[29] K LeFevre, R Agrawal, V Ercegovac, R Ramakrishnan, Y Xu, and D DeWitt Limiting disclosure in hippocratic databases In Proceedings of the 30th International Conference on Very Large Data Bases, VLDB '04, pages 108-119. VLDB Endowment, 2004.

[30] N Leone, G. Pfeifer, W Faber, T Eiter, G Gottlob, S Perri, and F Scarcello The dlv system for knowledge representation and reasoning. ACM Trans Comput Logıc, 7 499-562, July 2006

[31] N Leone, L Vicenzino, and G Terracina Dlvdb. Bridging the gap between asp systems and dbmss In Proceedings of the 7th International Conference on Logic Programming and Non-Monotonic Reasoning, volume 2923 of LNAI, pages 341-345 Springer, 2004.

[32] M Levene and G. Loizou Null inclusion dependencies in relational databases Information and Computation, 136(2) 67-108, 1994

[33] J W Lloyd. Foundations of logic programming, (2nd extended ed) SpringerVerlag New York, Inc, New York, NY, USA, 1987.

[34] I S Mumick, S. J Finkelstein, H Prrahesh, and R. Ramakrıshnan Magic is relevant SIGMOD Rec., 19 247-258, May 1990.

[35] L. E. Olson, C. A. Gunter, and P. Madhusudan A formal framework for reflective database access control policies In Proceedings of the 15th ACM Conference on Computer and Communıcatıons Securıty, CCS '08, pages 289-298, New York, NY, USA, 2008. ACM

[36] E Rahm and $\mathrm{H}$ H Do. Data cleaning Problems and current approaches IEEE Data Engineering Bulletın, 23·3-13, 2000.

[37] S Rizvı, A. Mendelzon, S Sudarshan, and P Roy Extending query rewriting techniques for fine-grained access control In Proceedings of the 2004 ACM SIGMOD International Conference on Management of Data, SIGMOD '04, pages 551-562, New York, NY, USA, 2004. ACM. 
[38] K A. Ross, D Srivastava, and S. Sudarshan Materialized view maintenance and integrity constraint checking. trading space for tıme. SIGMOD Rec., 25·447-458, June 1996

[39] G. Terracina, $\mathrm{N}$ Leone, V Lio, and C. Panetta Adding efficient data management to logic programming systems In Proceedings of the 16th International Symposium on Methodologies for Intelligent Systems (ISMIS 2006), volume 4203 of $L N C S$, pages 524-533. Springer, 2006.

[40] C Turker and M. Gertz. Semantic integrity support in SQL:1999 and commercial (object-)relational database management systems The VLDB Journal, 10.241269, December 2001.

[41] Y. Vassiliou. Null values in data base management a denotational semantics approach. In Proceedings of the 1979 ACM SIGMOD International Conference on Management of Data, SIGMOD '79, pages 162-169, New York, NY, USA, 1979. ACM

[42] T. Weibert. A framework for inference control in incomplete logic databases. In PhD. Thesis Technische Universitat Dortmund, 2008

[43] C Zaniolo Database relations with null values In Proceedings of the 1st ACM SIGACT-SIGMOD Symposium on Principles of Database Systems, PODS '82, pages 27-33, New York, NY, USA, 1982 ACM

[44] Z Zhang and A O Mendelzon Authorization views and conditional query containment In Proceedings of the 10th International Conference on Database Theory (ICDT 2005), volume 3363 of INCS, pages 259-273. Springer, 2005 


\section{Appendix A}

\section{Optimized Secrecy Programs}

Program 7. Optimized Logic Program for Query $\mathcal{Q}_{1}$.

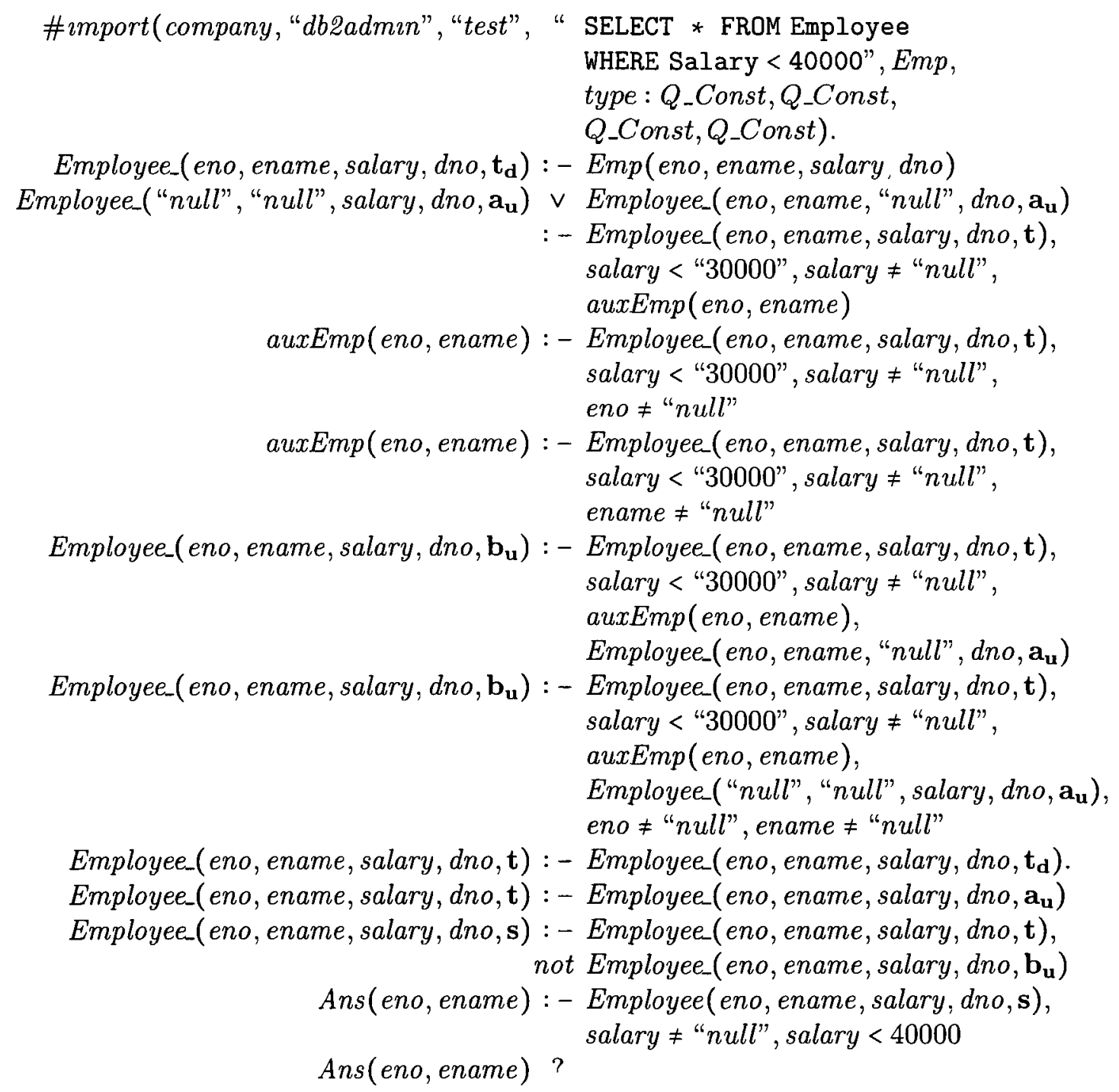


Program 8. Optimızed Logic Program for Query $\mathcal{Q}_{2}$.

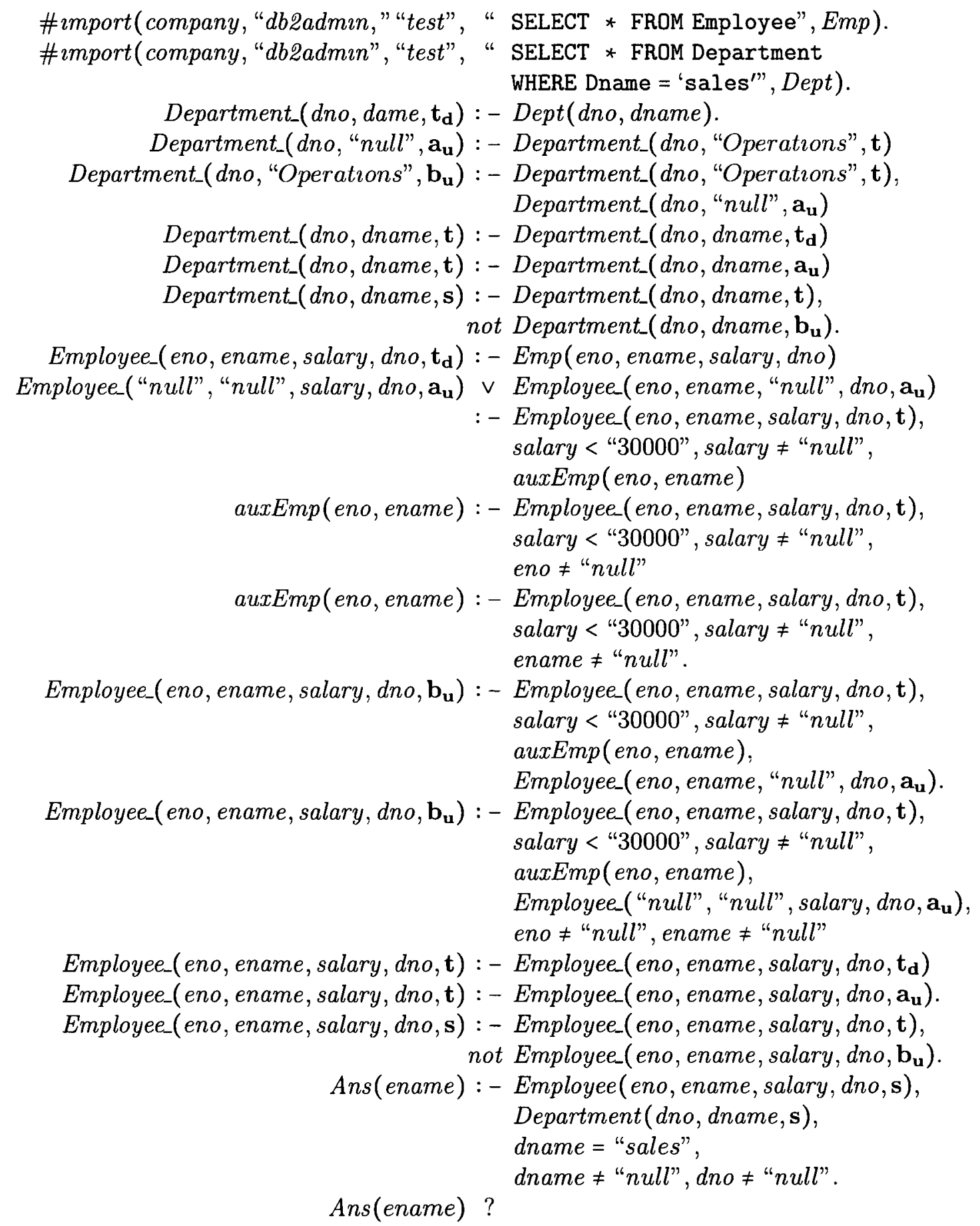


Program 9. Optimızed Log1c Program for Query $\mathcal{Q}_{3}$

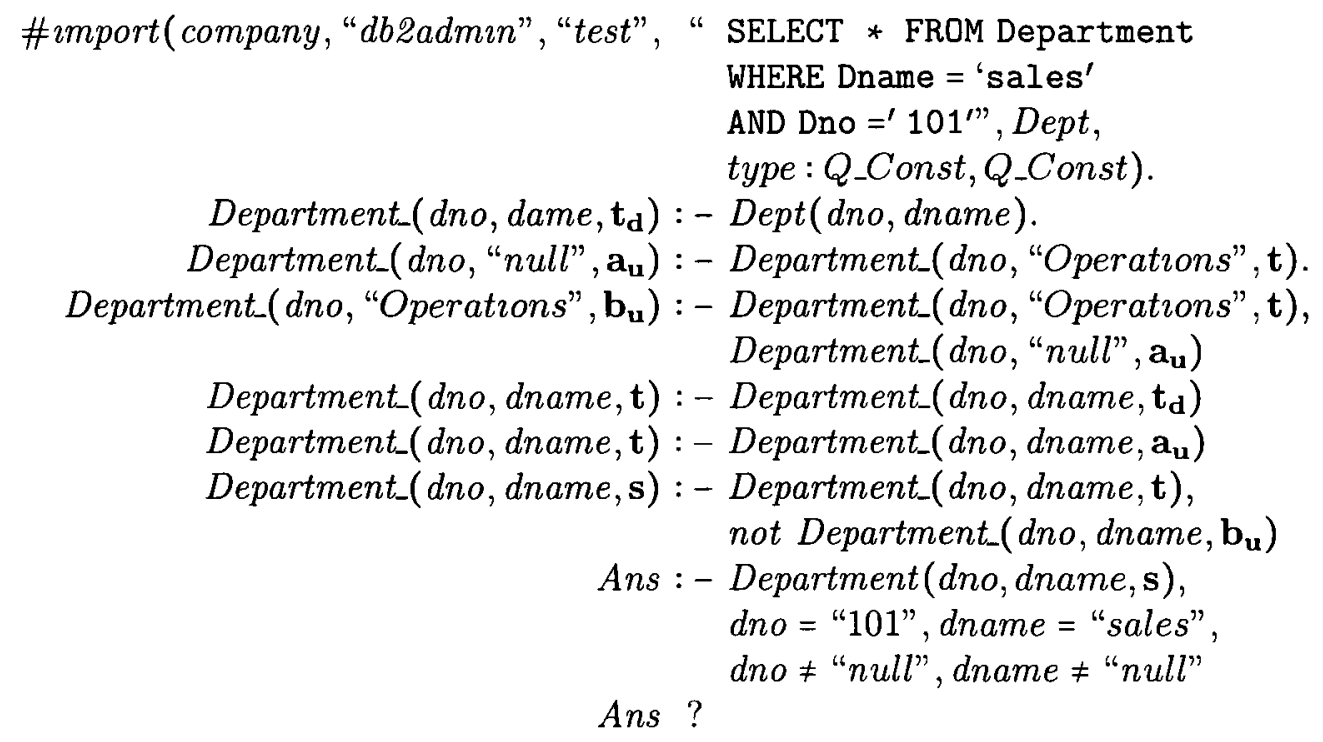

